\title{
Cyanamido-Bridged Diiridium Complex: a Reactive Building Block for Polynuclear Cyanamido Complexes
}

\author{
Hidenobu Kajitani, ${ }^{\dagger}$ Yoshiaki Tanabe, ${ }^{\ddagger}$ Shigeki Kuwata, ${ }^{\S}$ Masakazu Iwasaki, ${ }^{\natural}$ and \\ Youichi Ishii* ${ }^{* \ddagger}$
}

\author{
$\dagger$ Institute of Industrial Science, \\ The University of Tokyo, Komaba, Meguro-ku, Tokyo 153-8505, Japan \\ $\ddagger$ Department of Applied Chemistry, Faculty of Science and Engineering, \\ Chuo University, Kasuga, Bunkyo-ku, Tokyo 112-8551, Japan \\ $\S$ Department of Applied Chemistry, Graduate School of Science and Engineering, \\ Tokyo Institute of Technology, O-okayama, Meguro-ku, Tokyo 152-8552, Japan \\ I Department of Applied Chemistry, Faculty of Engineering, \\ Saitama Institute of Technology, Okabe, Saitama 369-0293, Japan
}

Table of Contents

Experimental and Spectroscopic Details

S2

Table S1. Kinetic Data for the Dimerization Reaction of 5

S5

Table S2. X-ray Crystallographic Data for 5, 6, 8d, 9·1.5 $\mathrm{C}_{6} \mathrm{H}_{6}$, and $\mathbf{1 0 a B P h}_{4} \cdot \mathrm{CH}_{2} \mathrm{Cl}_{2} \quad$ S6

Table S3. Positional Parameters and B(eq) for 5

S7

Table S4. Anisotropic Displacement Parameters for $\mathbf{5}$

S8

Table S5. Intramolecular Distances Involving the Nonhydrogen Atoms for $5 \quad$ S8

Table S6. Intramolecular Bond Angles Involving the Nonhydrogen Atoms for $5 \quad$ S9

$\begin{array}{ll}\text { Table S7. Positional Parameters and B(eq) for } \mathbf{6} & \text { S10 }\end{array}$

$\begin{array}{ll}\text { Table S8. Anisotropic Displacement Parameters for } 6 & \text { S11 }\end{array}$

Table S9. Intramolecular Distances Involving the Nonhydrogen Atoms for $6 \quad$ S12

Table S10. Intramolecular Bond Angles Involving the Nonhydrogen Atoms for $6 \quad$ S12

Table S11. Positional Parameters and B(eq) for $\mathbf{8 d}$

$\mathrm{S} 14$

Table S12. Anisotropic Displacement Parameters for 8d

S15

Table S13. Intramolecular Distances Involving the Nonhydrogen Atoms for 8d $\quad$ S15

Table S14. Intramolecular Bond Angles Involving the Nonhydrogen Atoms for 8d $\quad$ S15

Table S15. Positional Parameters and B(eq) for $\mathbf{9} \cdot 1.5 \mathrm{C}_{6} \mathrm{H}_{6}$

Table S16. Anisotropic Displacement Parameters for $\mathbf{9} \cdot 1.5 \mathrm{C}_{6} \mathrm{H}_{6}$

Table S17. Intramolecular Distances Involving the Nonhydrogen Atoms for $\mathbf{9} \cdot 1.5 \mathrm{C}_{6} \mathrm{H}_{6} \quad$ S21

Table S18. Intramolecular Bond Angles Involving the Nonhydrogen Atoms for $\mathbf{9} \cdot 1.5 \mathrm{C}_{6} \mathrm{H}_{6} \quad$ S22

Table S19. Positional Parameters and B(eq) for $\mathbf{1 0 a B P h} \cdot \mathrm{CH}_{2} \mathrm{Cl}_{2} \quad$ S24

Table S20. Anisotropic Displacement Parameters for $\mathbf{1 0 a B P h}_{4} \cdot \mathrm{CH}_{2} \mathrm{Cl}_{2} \quad \mathrm{~S} 25$

Table S21. Intramolecular Distances Involving the Nonhydrogen Atoms for $\mathbf{1 0 a B P h}_{4} \cdot \mathrm{CH}_{2} \mathrm{Cl}_{2} \quad \mathrm{~S} 26$

Table S22. Intramolecular Bond Angles Involving the Nonhydrogen Atoms for ${ }^{10 a B P h}{ }_{4}{ }^{\cdot} \mathrm{CH}_{2} \mathrm{Cl}_{2} \mathrm{~S} 27$

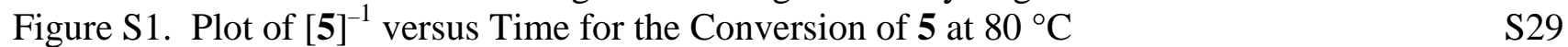

$\begin{array}{ll}\text { Figure S2. ORTEP Drawing of } 5 & \text { S30 }\end{array}$

$\begin{array}{ll}\text { Figure S3. ORTEP Drawing of } 6 & \text { S31 }\end{array}$

$\begin{array}{ll}\text { Figure S4. ORTEP Drawing of 8d } & \text { S32 }\end{array}$

$\begin{array}{ll}\text { Figure S5. ORTEP Drawing of } 9 & \text { S33 }\end{array}$

$\begin{array}{lr}\text { Figure S6. ORTEP Drawing of 10a } & \text { S34 }\end{array}$ 


\section{Experimental and Spectroscopic Details}

General Considerations. All reactions were carried out under a dry dinitrogen atmosphere using standard Schlenk techniques unless otherwise specified. [Cp*IrCl $]_{2}\left(\mathbf{1}, \mathrm{Cp}^{*}=\eta^{5}-\mathrm{C}_{5} \mathrm{Me}_{5}\right),{ }^{1}[\mathrm{RhCl}(\operatorname{cod})]_{2}(\operatorname{cod}=$ 1,5-cyclooctadiene), ${ }^{2}\left[\mathrm{CpRu}(\mathrm{MeCN})_{3}\right]\left(\mathrm{PF}_{6}\right)\left(\mathrm{Cp}=\eta^{5}-\mathrm{C}_{5} \mathrm{H}_{5}\right),{ }^{3}$ and $\left[\mathrm{PdCl}\left(\eta^{3}-\mathrm{C}_{3} \mathrm{H}_{5}\right)\right]_{2}{ }^{4}$ were prepared according to the literature methods. Solvents were dried by common procedures and degassed before use. Other reagents were commercially obtained and used as received. ${ }^{1} \mathrm{H}$ and ${ }^{13} \mathrm{C}\left\{{ }^{1} \mathrm{H}\right\}$ NMR spectra were recorded on a JEOL JNMLA-400, JEOL JNM-GSX-400 ( ${ }^{1} \mathrm{H}, 400 \mathrm{MHz} ;{ }^{31} \mathrm{P}, 162 \mathrm{MHz} ;{ }^{13} \mathrm{C}$, $\left.100 \mathrm{MHz}\right)$, or Varian Mercury-300 spectrometer $\left({ }^{1} \mathrm{H}, 300 \mathrm{MHz} ;{ }^{31} \mathrm{P}, 121.5 \mathrm{MHz}\right)$. IR spectra were recorded on a Shimadzu FT/IR-8100M or Jasco FT/IR-410 spectrometer. Elemental analyses were performed on a Perkin Elmer 2400 series II CHN analyzer. Amounts of the solvent molecules in the crystals were determined not only by elemental analyses but also by ${ }^{1} \mathrm{H}$ NMR spectroscopy.

Synthesis of $\left[\mathrm{Cp}^{*} \operatorname{Ir}\left(\mu_{2}-\mathrm{NCN}-\boldsymbol{N}, \boldsymbol{N}\right)\right]_{2}$ (5). Complex $1(1.0425 \mathrm{~g}, 1.31 \mathrm{mmol})$ and $\mathrm{Na}_{2} \mathrm{NCN}(229.5 \mathrm{mg}$, $2.67 \mathrm{mmol}$ ) were dissolved in methanol $(30 \mathrm{~mL})$ and stirred for $15 \mathrm{~min}$ at room temperature. The resulting dark red solution was dried in vacuo to give a dark red solid, which was dissolved in $\mathrm{CH}_{2} \mathrm{Cl}_{2}$-methanol (30:1, 1.0 mL) and passed through a column packed with $\mathrm{Al}_{2} \mathrm{O}_{3}$ (Merck; eluent, $\mathrm{CH}_{2} \mathrm{Cl}_{2}$-methanol 30:1). The first yellow band contains [Cp* $\left.\operatorname{Ir}\left(\mu_{3}-\mathrm{NCN}-N, N, N{ }^{\prime}\right)_{3}(\operatorname{IrCp} *)_{3}\left(\mu_{3}-\mathrm{NCN}-N, N, N\right)\right](3),{ }^{5}$ while the second dark red band eluted was collected and evaporated to dryness. Recrystallization from $\mathrm{CH}_{2} \mathrm{Cl}_{2}$-diethyl ether gave dark red needles of $\left[\mathrm{Cp} * \operatorname{Ir}\left(\mu_{2}-\mathrm{NCN}-N, N\right)\right]_{2}$ (5) (451.6 mg, 47\% yield). ${ }^{1} \mathrm{H}$ NMR $\left(\mathrm{CDCl}_{3}, \delta\right): 1.87$ (s, 30H, Cp*). ${ }^{13} \mathrm{C}\left\{{ }^{1} \mathrm{H}\right\} \mathrm{NMR}$ $\left(\mathrm{CDCl}_{3}, \delta\right): 9.7$ (s, $\mathrm{C}_{5} \mathrm{Me}_{5}$ ), 88.8 (s, $\left.\mathrm{C}_{5} \mathrm{Me}_{5}\right), 129.1$ (s, NCN). IR (KBr, cm $\left.{ }^{-1}\right): 2093$ (s, NCN), 1119 (m, NCN). Anal. Calcd for $\mathrm{C}_{22} \mathrm{H}_{30} \mathrm{Ir}_{2} \mathrm{~N}_{4}$ : C, 35.95; H, 4.11; N, 7.62. Found: C, 35.84; H, 4.04; N, 7.68.

Synthesis of $\left[\mathrm{Cp}^{*} \operatorname{Ir}(\mathrm{CO})\left(\mu_{2}-\mathrm{NCN}-\mathrm{N}, N\right)\left(\mu_{2}\right.\right.$-OCNCN)IrCp*] (6). When a $\mathrm{CH}_{2} \mathrm{Cl}_{2}$ solution (6 mL) of 5 (57.5 mg, $0.078 \mathrm{mmol}$ ) was placed under a carbon monoxide atmosphere at room temperature, its color immediately changed from dark red to orange. After stirring for $15 \mathrm{~min}$ at room temperature, the resulting solution was dried in vacuo to give a dark red solid. Recrystallization from toluene-hexane afforded [Cp* $\operatorname{Ir}(\mathrm{CO})\left(\mu_{2}-\mathrm{NCN}-N, N\right)\left(\mu_{2}\right.$-OCNCN)IrCp*] (6) as dark brown needles (50.8 mg, $0.064 \mathrm{mmol}, 82 \%$ yield). ${ }^{1} \mathrm{H}$ NMR $\left(\mathrm{CDCl}_{3}, \delta\right): 1.75,1.86$ (both s, 15H each, Cp*). IR (KBr, $\left.\mathrm{cm}^{-1}\right): 2186$ (m, NCN), 2107 (s, NCN), 2016 (s, CO), 1615 (s, CO), 1144 (m, NCN). Anal. Calcd for $\mathrm{C}_{24} \mathrm{H}_{30} \mathrm{Ir}_{2} \mathrm{~N}_{4} \mathrm{O}_{2}$ : C, 36.44; H, 3.82; N, 7.08. Found: C, 36.70; H, 3.79; N, 7.10.

Synthesis of [Cp* $\operatorname{Ir}(\mathrm{CNXy})\left(\mu_{2}-\mathrm{NCN}-N, N\right)\left(\mu_{2}\right.$-XyNCNCN)IrCp*] (7) and 7•0.5C $\mathrm{H}_{6} \mathrm{O}$. To a $\mathrm{CH}_{2} \mathrm{Cl}_{2}$ solution ( $5 \mathrm{~mL}$ ) of $\mathbf{5}$ (32.4 $\mathrm{mg}, 0.044 \mathrm{mmol})$ was added 2,6-dimethylphenyl isocyanide (11.9 $\mathrm{mg}, 0.091 \mathrm{mmol})$ at room temperature. The color of the solution changed immediately from dark red to yellow, and then slowly to dark purple. After $14 \mathrm{~h}$ of stirring at room temperature, the resulting solution was dried in vacuo to give a black oil. The residue was recrystallized from acetone-hexane to give dark purple crystals of [Cp* $\operatorname{Ir}(\mathrm{CNXy})\left(\mu_{2}-\mathrm{NCN}-\right.$ $N, N)\left(\mu_{2}\right.$-XyNCNCN)IrCp*] $0.5 \mathrm{C}_{3} \mathrm{H}_{6} \mathrm{O}\left(7 \cdot 0.5 \mathrm{C}_{3} \mathrm{H}_{6} \mathrm{O}, \mathrm{Xy}=2,6\right.$-dimethylphenyl). The acetone molecule in the crystals was lost by drying in vacuo to yield 7 (18.0 mg, $0.018 \mathrm{mmol}, 41 \%$ yield) as a powder. ${ }^{1} \mathrm{H}$ NMR $\left(\mathrm{C}_{6} \mathrm{D}_{6}\right.$, d): 1.42, 1.90 (both s, 15H each, Cp*), 2.20 (s, 3H, NCNCNC $\mathrm{H}_{3} \mathrm{Me}_{2}$ ), 2.30 (s, 6H, $\mathrm{CNC}_{6} \mathrm{H}_{3} \mathrm{Me}_{2}$ ), 2.66 (s, 3H, $\mathrm{NCNCNC}_{6} \mathrm{H}_{3} \mathrm{Me}_{2}$ ), 6.65 (d, 2H, $J=7.2 \mathrm{~Hz}, \mathrm{C}_{6} \mathrm{H}_{3}$ ), 6.70 (t, $1 \mathrm{H}, J=7.2 \mathrm{~Hz}, \mathrm{C}_{6} \mathrm{H}_{3}$ ), 7.01 (t, $1 \mathrm{H}, J=7.2 \mathrm{~Hz}, \mathrm{C}_{6} \mathrm{H}_{3}$ ), 7.11 (d, $\left.1 \mathrm{H}, J=7.2 \mathrm{~Hz}, \mathrm{C}_{6} \mathrm{H}_{3}\right), 7.26$ (d, $\left.1 \mathrm{H}, J=7.2 \mathrm{~Hz}, \mathrm{C}_{6} \mathrm{H}_{3}\right) . \quad \mathrm{IR}\left(\mathrm{KBr}, \mathrm{cm}^{-1}\right): 2159(\mathrm{~m}, \mathrm{NCN}), 2132(\mathrm{~s}, \mathrm{NCN})$, 2090 (s, CN), 1553 (s, CN), 1195 (m, NCN). Anal. Calcd for $\mathrm{C}_{40} \mathrm{H}_{48} \mathrm{Ir}_{2} \mathrm{~N}_{6}$ : C, 48.17; H, 4.85; N, 8.43. Found: C, 47.78; H, 4.95; N, 8.26.

Synthesis of [Cp* $\operatorname{Ir}\left(\mathbf{P P h}_{3}\right)\left(\mu_{2}-\mathbf{N C N}-\boldsymbol{N}, \boldsymbol{N}\right)_{2} \mathbf{I r C p}$ ] (8a). To a $\mathrm{CH}_{2} \mathrm{Cl}_{2}$ solution $(10 \mathrm{~mL})$ of 5 (78.1 mg, $0.106 \mathrm{mmol})$ was added triphenylphosphine $(29.7 \mathrm{mg}, 0.113 \mathrm{mmol})$ at room temperature. After stirring for $3 \mathrm{~h}$, the solution was evaporated to $\mathrm{ca}$. $3 \mathrm{~mL}$. Recrystallization from $\mathrm{CH}_{2} \mathrm{Cl}_{2}$-diethyl ether gave dark purple needles of $\left[\mathrm{Cp} * \operatorname{Ir}\left(\mathrm{PPh}_{3}\right)\left(\mu_{2}-\mathrm{NCN}-N, N\right)_{2} \operatorname{IrCp} *\right]$ (8a) $\left(81.4 \mathrm{mg}, 0.082 \mathrm{mmol}, 77 \%\right.$ yield). ${ }^{1} \mathrm{H}$ NMR $\left(\mathrm{CDCl}_{3}, \delta\right): 1.40$ (d, $15 \mathrm{H}, J=2.0 \mathrm{~Hz}, \mathrm{Cp} *), 1.55$ (s, 15H, Cp*), 7.28-7.41 (m, 9H, Ph), 7.53-7.61 (m, 6H, Ph). ${ }^{31} \mathrm{P}\left\{{ }^{1} \mathrm{H}\right\} \mathrm{NMR}\left(\mathrm{CDCl}_{3}\right.$, d): 18.7 (s). IR (KBr, $\mathrm{cm}^{-1}$ ): 2064 (s, NCN), 1181 (m, NCN). Anal. Calcd for $\mathrm{C}_{40} \mathrm{H}_{45} \mathrm{Ir}_{2} \mathrm{~N}_{4} \mathrm{P}: \mathrm{C}, 48.18 ; \mathrm{H}, 4.55 ; \mathrm{N}$, 5.62. Found: C, 47.78; H, 4.52; N, 5.55 .

Synthesis of $\left[\mathbf{C p}^{*} \operatorname{Ir}\left\{\mathbf{P}(\boldsymbol{p}-\mathrm{Tol})_{3}\right\}\left(\mu_{2}-\mathrm{NCN}-\boldsymbol{N}, \boldsymbol{N}\right)_{2} \operatorname{IrCp} \mathbf{p}^{*}\right.$ (8b). This compound was prepared from 5 (30.4 $\mathrm{mg}, 0.041 \mathrm{mmol})$ and tri $(p$-tolyl)phosphine $(13.0 \mathrm{mg}, 0.043 \mathrm{mmol})$ in a manner analogous to that described for 8a. Recrystallization from toluene-hexane gave dark green crystals of $\left[\mathrm{Cp} * \operatorname{Ir}\left\{\mathrm{P}(p-\mathrm{Tol})_{3}\right\}\left(\mu_{2}-\mathrm{NCN}-N, N\right)_{2} \operatorname{IrCp}{ }^{*}\right](\mathbf{8 b})$ (18.2 mg, $0.018 \mathrm{mmol}, 42 \%$ yield). ${ }^{1} \mathrm{H} \mathrm{NMR}\left(\mathrm{CDCl}_{3}, \delta\right.$ ): 1.40 (d, 15H, $\left.J=2.0 \mathrm{~Hz}, \mathrm{Cp} *\right), 1.55$ (s, 15H, Cp*), 2.36 (s, 9H, Me of $p$-Tol), 7.17 (d, $6 \mathrm{H}, J=8.1 \mathrm{~Hz}, m-\mathrm{H}$ of Tol), 7.44 (dd, $6 \mathrm{H},{ }^{3} J_{\mathrm{PH}}=11.1 \mathrm{~Hz},{ }^{3} J_{\mathrm{HH}}=8.1 \mathrm{~Hz}, o-\mathrm{H}$ of Tol). ${ }^{31} \mathrm{P}\left\{{ }^{1} \mathrm{H}\right\}$ NMR $\left(\mathrm{CDCl}_{3}, \delta\right): 17.0$ (s). IR (KBr, $\left.\mathrm{cm}^{-1}\right)$ : 2060 (s, NCN), 1179 (m, NCN). Anal. Calcd for $\mathrm{C}_{43} \mathrm{H}_{51} \mathrm{Ir}_{2} \mathrm{~N}_{4} \mathrm{P}$ : C, 49.69; H, 4.95; N, 5.39. Found: C, 49.68; H, 4.92; N, 5.35. 


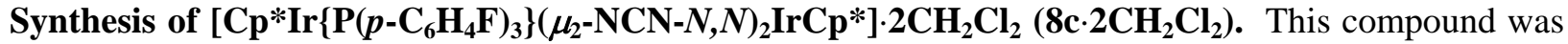
prepared from 5 (56.8 mg, $0.077 \mathrm{mmol}$ ) and tris(4-fluorophenyl)phosphine (24.4 $\mathrm{mg}, 0.077 \mathrm{mmol})$ in a manner analogous to that described for 8a. Recrystallization from $\mathrm{CH}_{2} \mathrm{Cl}_{2}$-hexane gave dark purple crystals of $\left[\mathrm{Cp} * \operatorname{Ir}\left\{\mathrm{P}\left(p-\mathrm{C}_{6} \mathrm{H}_{4} \mathrm{~F}\right)_{3}\right\}\left(\mu_{2}-\mathrm{NCN}-N, N\right)_{2} \mathrm{IrCp} *\right] \cdot 2 \mathrm{CH}_{2} \mathrm{Cl}_{2}\left(\mathbf{8 c} \cdot 2 \mathrm{CH}_{2} \mathrm{Cl}_{2}\right)(78.7 \mathrm{mg}, 0.064 \mathrm{mmol}, 83 \%$ yield $) .{ }^{1} \mathrm{H}$ NMR $\left(\mathrm{CDCl}_{3}, \delta\right.$ ): 1.42 (d, 15H, $J=2.0 \mathrm{~Hz}, \mathrm{Cp}^{*}$ ), 1.57 (s, 15H, Cp*), 5.30 (s, 4H, $\mathrm{CH}_{2} \mathrm{Cl}_{2}$ ), 7.11 (pseudo t, 6H, $J=8.2$ $\mathrm{Hz}, m-\mathrm{H}$ of $\left.\mathrm{C}_{6} \mathrm{H}_{4} \mathrm{~F}\right), 7.54$ (dd, $6 \mathrm{H},{ }^{3} J_{\mathrm{PH}}=15.2 \mathrm{~Hz},{ }^{3} J_{\mathrm{HH}}=8.6 \mathrm{~Hz}, o-\mathrm{H}$ of $\left.\mathrm{C}_{6} \mathrm{H}_{4} \mathrm{~F}\right) .{ }^{31} \mathrm{P}\left\{{ }^{1} \mathrm{H}\right\} \mathrm{NMR}\left(\mathrm{CDCl}_{3}, \delta\right): 18.3$ (s). IR (KBr, cm ${ }^{-1}$ ): 2065 (s, NCN), 1162 (m, NCN). Anal. Calcd for $\mathrm{C}_{42} \mathrm{H}_{46} \mathrm{Cl}_{4} \mathrm{~F}_{3} \mathrm{Ir}_{2} \mathrm{~N}_{4} \mathrm{P}: \mathrm{C}, 41.31 ; \mathrm{H}, 3.80 ; \mathrm{N}$, 4.59. Found: C, $41.21 ; \mathrm{H}, 3.79 ; \mathrm{N}, 4.60$.

Synthesis of $\left[\mathbf{C p} * \operatorname{Ir}\left(\mathbf{P M e}_{3}\right)\left(\mu_{2}-\mathbf{N C N}-N, N\right)_{2} \operatorname{IrCp} *\right]$ (8d). This compound was prepared from 5 (31.3 mg, $0.043 \mathrm{mmol}$ ) and trimethylphosphine (3.4 mg, $0.045 \mathrm{mmol}$ ) in a manner analogous to that described for 8a. Recrystallization from benzene-hexane gave dark purple needles of $\left[\mathrm{Cp} * \operatorname{Ir}\left(\mathrm{PMe}_{3}\right)\left(\mu_{2}-\mathrm{NCN}-N, N\right)_{2} \operatorname{IrCp} *\right]$ (8d) (11.9 mg, 0.015 mmol, 34\% yield). ${ }^{1} \mathrm{H}$ NMR $\left(\mathrm{C}_{6} \mathrm{D}_{6}, \delta\right.$ ): 1.28 (d, 9H, $\left.J=10.8 \mathrm{~Hz}, \mathrm{PMe}_{3}\right), 1.46$ (s, 15H, Cp*), 1.61 (s, 15H, Cp*). ${ }^{31} \mathrm{P}\left\{{ }^{1} \mathrm{H}\right\}$ NMR $\left(\mathrm{C}_{6} \mathrm{D}_{6}, \delta\right):-23.2$ (s). IR (KBr, $\left.\mathrm{cm}^{-1}\right): 2062$ (s, NCN), 1181 (m, NCN). Anal. Calcd for $\mathrm{C}_{25} \mathrm{H}_{39} \mathrm{Ir}_{2} \mathrm{~N}_{4} \mathrm{P}$ : C, 37.02; H, 4.85; N, 6.91. Found: C, 36.85; H, 4.82; N, 7.03.

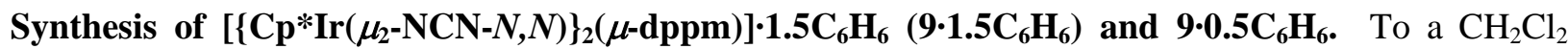
solution (5 mL) of 5 (29.1 mg, $0.040 \mathrm{mmol}$ ) was added bis(diphenylphosphino)methane (16.7 mg, $0.043 \mathrm{mmol}$ ) at room temperature. The color of the solution immediately changed to yellow. After stirring for $1 \mathrm{~h}$, the solution was dried in vacuo to afford yellow residue, which was extracted with benzene and recrystallized from benzene-hexane to yield dark red block crystals of $\left[\left\{\mathrm{Cp} * \operatorname{Ir}\left(\mu_{2}-\mathrm{NCN}-N, N\right)\right\}_{2}(\mu\right.$-dppm) $] \cdot 1.5 \mathrm{C}_{6} \mathrm{H}_{6}\left(9 \cdot 1.5 \mathrm{C}_{6} \mathrm{H}_{6}, \mathrm{dppm}\right.$ = bis(diphenylphosphino)methane) (37.2 mg, $0.030 \mathrm{mmol}, 76 \%$ yield). The crystals give off a part of benzene by drying in vacuo for $8 \mathrm{~h}$ at $90{ }^{\circ} \mathrm{C}$ to afford a yellow powder with the experimental formula of $9 \cdot 0.5 \mathrm{C}_{6} \mathrm{H}_{6} \cdot{ }^{1} \mathrm{H}$ NMR $\left(\mathrm{CDCl}_{3}, \delta\right): 1.45$ (s, 30H, Cp*), 3.84 (t, 2H, $\left.J=11.4 \mathrm{~Hz}, \mathrm{CH}_{2}\right), 7.18-7.29$ (m, 12H, Ph), 7.36 (s, 3H, $\left.\mathrm{C}_{6} \mathrm{H}_{6}\right), 7.42-$ $7.52(\mathrm{~m}, 8 \mathrm{H}, \mathrm{Ph}) .{ }^{31} \mathrm{P}\left\{{ }^{1} \mathrm{H}\right\}$ NMR $\left(\mathrm{CDCl}_{3}, \delta\right): 7.8$ (s). IR $\left(\mathrm{KBr}, \mathrm{cm}^{-1}\right): 2049$ (s, NCN). Anal. Calcd for $\mathrm{C}_{50} \mathrm{H}_{55} \mathrm{Ir}_{2} \mathrm{~N}_{4} \mathrm{P}_{2}$ : C, 51.84; H, 4.79; N, 4.84. Found: C, 51.74; H, 4.85; N, 4.74.

Synthesis of $\left[\left(C p^{*} I r\right)_{2}\{\operatorname{Rh}(\operatorname{cod})\}\left(\mu_{3}-\mathrm{NCN}-N, N, N\right)_{2}\right]\left(\mathrm{BPh}_{4}\right)(10 \mathrm{aBPh})$ and $10 \mathrm{aBPh}_{4} \cdot \mathrm{CH}_{2} \mathrm{Cl}_{2}$. To a $\mathrm{CH}_{2} \mathrm{Cl}_{2}$ solution $(30 \mathrm{~mL})$ of $5(247.8 \mathrm{mg}, 0.337 \mathrm{mmol})$ was added an acetone solution of [Rh(cod)(acetone) $\left.{ }_{n}\right]^{+}$, which was prepared in situ from $[\mathrm{RhCl}(\mathrm{cod})]_{2}(84.0 \mathrm{mg}, 0.170 \mathrm{mmol})$ and AgOTf (OTf = trifluoromethanesulfonate) $(87.0 \mathrm{mg}, 0.339 \mathrm{mmol})$ in acetone $(10 \mathrm{~mL})$. The mixture was stirred for $12 \mathrm{~h}$ at room temperature, and the resultant dark red solution was dried in vacuo. The residue was dissolved in methanol (30 $\mathrm{mL}$ ) containing $\mathrm{NaBPh}_{4}(\mathrm{ca} .170 \mathrm{mg}, 0.50 \mathrm{mmol}$ ). The mixture was stirred for $12 \mathrm{~h}$ at room temperature to form a red precipitate, which was collected by filtration, dried in vacuo, and extracted with $\mathrm{CH}_{2} \mathrm{Cl}_{2}$. Recrystallization from $\mathrm{CH}_{2} \mathrm{Cl}_{2}$-methanol yielded dark red plate crystals of $\left[(\mathrm{Cp} * \mathrm{Ir})_{2}\{\mathrm{Rh}(\mathrm{cod})\}\left(\mu_{3}-\mathrm{NCN}-N, N, N\right)_{2}\right]\left(\mathrm{BPh}_{4}\right) \cdot \mathrm{CH}_{2} \mathrm{Cl}_{2}$ $\left(\mathbf{1 0 a B P h}{ }_{4} \cdot \mathrm{CH}_{2} \mathrm{Cl}_{2}\right)$, the $\mathrm{CH}_{2} \mathrm{Cl}_{2}$ molecule of which was lost by drying in vacuo to give a red powder of $\mathbf{1 0 a B P h}_{4}$ (369.3 mg, $0.292 \mathrm{mmol}, 87 \%$ yield). ${ }^{1} \mathrm{H}$ NMR ( $\mathrm{CDCl}_{3}, \delta$ ): 2.03 (s, 30H, Cp*), 2.34-2.38 (m, 8H, cod), 3.90-3.96 (m, 4H, cod), 6.86 (t, 4H, $J=7.2 \mathrm{~Hz}, \mathrm{Ph}), 7.01$ (t, 8H, $J=7.2 \mathrm{~Hz}, \mathrm{Ph}), 7.38-7.44(\mathrm{~m}, 8 \mathrm{H}, \mathrm{Ph})$. IR $\left(\mathrm{KBr}, \mathrm{cm}^{-1}\right)$ : 2159 (s, NCN), 1138 (m, NCN). Anal. Calcd for $\mathrm{C}_{54} \mathrm{H}_{62} \mathrm{BIr}_{2} \mathrm{~N}_{4} \mathrm{Rh}$ : C, 51.26; H, 4.94; N, 4.43. Found: C, 50.97; H, 4.92; N, 4.55 .

Synthesis of $\left[\left(\mathrm{Cp}^{*} \mathrm{Ir}\right)_{2}(\mathbf{C p R u})\left(\mu_{3}-\mathrm{NCN}-N, \boldsymbol{N}, \boldsymbol{N}\right)_{2}\right]\left(\mathbf{B P h}_{4}\right)\left(\mathbf{1 0 b B P h}_{4}\right)$. To a $\mathrm{CH}_{2} \mathrm{Cl}_{2}$ solution (8 mL) of 5 (41.6 mg, $0.057 \mathrm{mmol})$ was added $\left[\mathrm{CpRu}(\mathrm{MeCN})_{3}\right]\left(\mathrm{PF}_{6}\right)(24.5 \mathrm{mg}, 0.056 \mathrm{mmol})$ and stirred for $12 \mathrm{~h}$ at room temperature, while the dark blue solution was gradually changed to a dark brown suspension. The mixture was dried in vacuo and dissolved in acetonitrile $(8 \mathrm{~mL})$ containing $\mathrm{NaBPh}_{4}(\mathrm{ca} .170 \mathrm{mg}, 0.50 \mathrm{mmol})$. The mixture was stirred for $12 \mathrm{~h}$ at room temperature, dried in vacuo, and extracted with $\mathrm{CH}_{2} \mathrm{Cl}_{2}$. Recrystallization from $\mathrm{CH}_{2} \mathrm{Cl}_{2}-$ methanol yielded red crystals of [(Cp*Ir) $\left.2(\mathrm{RuCp})\left(\mu_{3}-\mathrm{NCN}-N, N, N\right)_{2}\right]\left(\mathrm{BPh}_{4}\right)\left(\mathbf{1 0 b B P h}_{4}\right)(52.0 \mathrm{mg}, 0.043$ mmol, 75\% yield). ${ }^{1} \mathrm{H}$ NMR $\left(\mathrm{CD}_{2} \mathrm{Cl}_{2}, \delta\right.$ ): 1.88 (s, 30H, Cp*), 4.83 (s, 5H, Cp), 6.86 (t, 4H, $\left.J=6.9 \mathrm{~Hz}, \mathrm{Ph}\right), 7.01$ (t, 8H, $J=6.9 \mathrm{~Hz}, \mathrm{Ph}), 7.24-7.35$ (m, 8H, Ph). IR (KBr, cm $\left.{ }^{-1}\right): 2170$ (s, NCN), 1139 (m, NCN). Anal. Calcd for $\mathrm{C}_{51} \mathrm{H}_{55} \mathrm{BIr}_{2} \mathrm{~N}_{4} \mathrm{Ru}$ : C, 50.20; H, 4.54; N, 4.59. Found: C, 50.43; H, 4.51; N, 4.52.

Synthesis of $\left[(\mathbf{C p} * \mathbf{I r})_{2}\left\{\mathbf{P d}\left(\eta^{3}-\mathrm{C}_{3} \mathrm{H}_{5}\right)\right\}\left(\mu_{3}-\mathrm{NCN}-\boldsymbol{N}, \boldsymbol{N}, \boldsymbol{N}\right)_{2}\right](\mathbf{O T f})$ (10cOTf). To an acetone solution (5 $\mathrm{mL}$ ) of 5 (50.6 mg, $0.069 \mathrm{mmol})$ was added an acetone solution of $\left[\mathrm{Pd}\left(\eta^{3}-\mathrm{C}_{3} \mathrm{H}_{5}\right)(\text { acetone })_{n}\right]^{+}$, which was prepared in situ from $\left[\mathrm{PdCl}\left(\eta^{3}-\mathrm{C}_{3} \mathrm{H}_{5}\right)\right]_{2}(12.9 \mathrm{mg}, 0.035 \mathrm{mmol})$ and AgOTf $(17.6 \mathrm{mg}, 0.069 \mathrm{mmol})$ in acetone $(3 \mathrm{~mL})$. The mixture was stirred for $5 \mathrm{~h}$ at room temperature, and the resultant dark red solution was dried in vacuo and recrystallized from acetone-hexane to yield red crystals of $\left[(\mathrm{Cp} * \operatorname{Ir})_{2}\left\{\mathrm{Pd}\left(\eta^{3}-\mathrm{C}_{3} \mathrm{H}_{5}\right)\right\}\left(\mu_{3}-\mathrm{NCN}-N, N, N\right)_{2}\right](\mathrm{OTf})$ (10cOTf) (52.5 mg, $0.051 \mathrm{mmol}, 74 \%$ yield). ${ }^{1} \mathrm{H} \mathrm{NMR}\left(\mathrm{CDCl}_{3}, \delta\right): 1.94$ (s, 30H, Cp*), 3.26 (d, $2 \mathrm{H}, J=12.3 \mathrm{~Hz}$, $\mathrm{CH}_{2}$ ), 4.19-4.35 (m, 2H, CH ), 5.85-5.95 (m, 1H, CH). IR (KBr, cm $\left.{ }^{-1}\right)$ : 2145 (s, NCN), 1151 (m, NCN). Anal. Calcd for $\mathrm{C}_{26} \mathrm{H}_{35} \mathrm{~F}_{3} \mathrm{Ir}_{2} \mathrm{~N}_{4} \mathrm{O}_{3}$ PdS: C, 30.27; H, 3.42; N, 5.43. Found: C, 30.40; H, 3.31; N, 5.23. 
Kinetic Study of the Dimerization Reaction of 5. Complex 5 (50.2 mg, $0.068 \mathrm{mmol}$ ) was dissolved in 1-propanol $\left(5.0 \mathrm{~mL}, 14 \mathrm{mmol} \mathrm{L}{ }^{-1}\right)$ at room temperature and then was quickly heated to $80{ }^{\circ} \mathrm{C}$ in an oil bath. In order to monitor the dimerization of 5 into the cubane-type complex [Cp* $\left.\operatorname{Ir}\left(\mu_{3}-\mathrm{NCN}-N, N, N\right)\right]_{4}(4), c a .0 .1 \mathrm{~mL}$ of the solution was taken by a syringe every 10 minutes, dried in vacuo, and the relative intensities of the $\mathrm{Cp}^{*}$ protons were measured by ${ }^{1} \mathrm{H}$ NMR $\left(\mathrm{CDCl}_{3}\right)$.

The reaction was also performed for solutions with the concentration of 3.5, 6.8, and $27 \mathrm{mmol} / \mathrm{L}$ at $80{ }^{\circ} \mathrm{C}$ $(5,14 \mathrm{mmol} / \mathrm{L})$. Kinetic data are summarized in Table S1. The plot of $[5]^{-1}$ versus time at $80{ }^{\circ} \mathrm{C}$ (Figure S1) indicates that the reaction obeys second-order kinetics.

X-ray Diffraction Studies. Diffraction data for 5, 6, and $10 \mathrm{aBPh} \cdot \mathrm{CH}_{2} \mathrm{Cl}_{2}$ were collected on a Rigaku AFC-7R (5 and 6) or AFC-7S $\left(\mathbf{1 0 a B P h} \cdot \cdot \mathrm{CH}_{2} \mathrm{Cl}_{2}\right)$ four-circle automated diffractometer with graphitemonochromated Mo $K \alpha$ radiation $(\lambda=0.71069 \AA)$ at $21{ }^{\circ} \mathrm{C}$ using the $\omega(5)$ or $\omega-2 \theta$ scan (6 and $10 \mathrm{aBPh} 4{ }_{4} \mathrm{CH}_{2} \mathrm{Cl}_{2}$ ) technique. Cell parameters were determined by least-squares refinement of 25 machinecentered reflections with $39.65<2 \theta<39.98^{\circ}$ for $\mathbf{5}$, $39.72<\mathrm{a} 2 \theta<40.01^{\circ}$ for $\mathbf{6}$, and $37.17<2 \theta<39.75^{\circ}$ for $10 \mathrm{aBPh} \cdot \mathrm{CH}_{2} \mathrm{Cl}_{2}$.

Diffraction data for $\mathbf{8 d}$ and $\mathbf{9} \cdot 1.5 \mathrm{C}_{6} \mathrm{H}_{6}$ were collected on a Rigaku RAXIS-RAPID imaging plate area detector with graphite-monochromated Mo K $\alpha$ radiation $(\lambda=0.71069 \AA)$ at $-165(\mathbf{8 d})$ or $21\left(\mathbf{9} \cdot 1.5 \mathrm{C}_{6} \mathrm{H}_{6}\right){ }^{\circ} \mathrm{C}$. Cell parameters were refined using 22586 and 20891 reflections for $\mathbf{8 d}$ and $\mathbf{9} \cdot 1.5 \mathrm{C}_{6} \mathrm{H}_{6}$, respectively.

No significant decay was observed over the course of data collection for $\mathbf{5 , 6}, \mathbf{8 d}$, and $\mathbf{9} \cdot 1.5 \mathrm{C}_{6} \mathrm{H}_{6}$, while a slight decay (2.52\%) was observed for $10 \mathrm{aBPh}_{4} \cdot \mathrm{CH}_{2} \mathrm{Cl}_{2}$, and a linear correction factor was applied to account for this phenomenon. Intensity data were corrected for empirical absorptions ( $\psi$ scans for 5, 6, and $\mathbf{1 0 a B P h} \cdot \mathrm{CH}_{2} \mathrm{Cl}_{2}$; spherical scans using the program $\mathrm{ABSCOR}^{6}$ for $\mathbf{8 d}$ and $\mathbf{9} \cdot 1.5 \mathrm{C}_{6} \mathrm{H}_{6}$ ) and for Lorentz and polarization effects. A correction for secondary extinction was further applied for $\mathbf{1 0}$ (coefficient, 38.133). ${ }^{7}$

The structure solution and refinements were carried out by using the CrystalStructure package. ${ }^{8}$ The positions of the non-hydrogen atoms were determined by heavy atom Patterson methods (SHELX-97 ${ }^{9}$ for 5, 6, and $\mathbf{9} \cdot 1.5 \mathrm{C}_{6} \mathrm{H}_{6}$; PATTY $^{10}$ for $\mathbf{8 d}$ and $\mathbf{1 0 a B P h}{ }_{4} \cdot \mathrm{CH}_{2} \mathrm{Cl}_{2}$ ) and subsequent Fourier syntheses (DIRDIF99). ${ }^{11}$ All the non-hydrogen atoms were refined on $F_{\mathrm{o}}(I>3 \sigma(I))$ by full-matrix least squares techniques with anisotropic thermal parameters, while all the hydrogen atoms were placed at the calculated positions with fixed isotropic parameters. The atomic scattering factors were taken from ref. 12. Anomalous dispersion effects were included in $F_{\mathrm{c} .}{ }^{13}$ The values of $\Delta f$ ' and $\Delta f$ " were taken from ref. 14 . The maximum and minimum residual peaks on the final difference Fourier maps were 5.93/-3.41, 1.21/-1.35, 3.60/-2.21 1.71/-1.67, and 2.68/-1.58 $\mathrm{e}^{-} / \AA^{3}$ for 5, 6, 8d, $\mathbf{9} \cdot 1.5 \mathrm{C}_{6} \mathrm{H}_{6}$, and $10 \mathrm{aBPh}_{4} \cdot \mathrm{CH}_{2} \mathrm{Cl}_{2}$, respectively. Details of the $\mathrm{X}$-ray diffraction study are summarized in Table S2. Positional and anisotropic displacement parameters, bond distances and angles for complexes 5, 6, 8d, $\mathbf{9} \cdot 1.5 \mathrm{C}_{6} \mathrm{H}_{6}$, and $10 \mathrm{aBPh}{ }_{4} \cdot \mathrm{CH}_{2} \mathrm{Cl}_{2}$ are summarized in Tables S3-S22, and their ORTEP drawings are shown in Figures S2-S6.

\section{References}

(1) White, C.; Yates, A.; Maitlis, P. M.; Heinekey, D. M. Inorg. Synth. 1992, 29, 228-234.

(2) Giordani, G.; Crabtree, R. H. Inorg. Synth. 1979, 19, 218-220.

(3) (a) Bennett, M. A.; Smith, A. K. J. Chem. Soc., Dalton Trans. 1974, 233-241. (b) Trost, B. M.; Older, C. M. Organometallics 2002, 21, 2544-2546.

(4) Tatsuno, Y.; Yoshida, T.; Otsuka, S. Inorg. Synth. 1990, 28, 342-345.

(5) Tanabe, Y.; Kuwata, S.; Ishii, Y. J. Am. Chem. Soc. 2002, 124, 6528-6529.

(6) Higashi, T. ABSCOR: Empirical Absorption Correction based on Fourier Series Approximation; Rigaku Corp.: Tokyo, Japan, 1995.

(7) Larson, A. C. In Crystallographic Computing; Proceedings of an International Summer School organized by The Commission on Crystallographic Computing of the International Union of Crystallography and held in Ottawa, 4-11 August 1969; Ahmed, F. R., Hall, S. R., Huber, C. P., Eds.; Munksgaard: Copenhagen, Denmark, 1970; pp. 291-295.

(8) (a) CrystalStructure 3.60: Single Crystal Structure Analysis Software; Rigaku Corp: Tokyo, Japan and MSC: The Woodlands, TX, 2000-2004. (b) Watkin, D. J.; Prout, C. K.; Carruthers, J. R.; Betteridge, P. W. CRYSTALS Issue 10; Chemical Crystallography Laboratory: Oxford, UK, 1996.

(9) Sheldrick, G. M. SHELX-97: Program for the refinement of crystal structure; University of Göttingen: Göttingen, Germany, 1997. 
(10) Beurskens, P. T.; Admiraal, G.; Beurskens, G.; Bosman, W. P.; Garcia-Granda, S.; Gould, R. O.; Smits, J. M. M.; Smykalla, C. PATTY: The DIRDIF program system; Technical Report of the Crystallography Laboratory, University of Nijmengen: Nijmengen, The Netherlands, 1992.

(11) Beurskens, P. T.; Admiraal, G.; Beurskens, G.; Bosman, W. P.; de Gelder. R.; Israel, R.; Smits, J. M. M. DIRDIF99: The DIRDIF-99 program system; Technical Report of the Crystallography Laboratory, University of Nijmegen: Nijmengen, The Netherlands, 1999.

(12) Cromer, D. T.; Waber, J. T. International Tables for X-ray Crystallography; Ibers, J. A., Hamilton, W. C. Eds.; Kynoch Press: Birmingham, England, 1974; Vol. IV., Table 2.2 A.

(13) Ibers, J. A.; Hamilton, W. C. Acta Crystallogr. 1964, 17, 781.

(14) (a) Creagh, D. C.; McAuley, W. J. International Tables for X-ray Crystallography; Wilson, A. J. C. Ed.; Kluwer Academic Publishers: Boston, MA, 1992; Vol. C. Table 4.2.6.8. (b) Creagh, D. C.; Hubbell, J. H. International Tables for X-ray Crystallography; Wilson, A. J. C. Ed.; Kluwer Academic Publishers: Boston, MA, 1992; Vol. C. Table 4.2.4.3.

Table S1. Kinetic Data for the Dimerization Reaction of $\mathbf{5}$.

\begin{tabular}{lllll}
\hline run & $T,{ }^{\circ} \mathrm{C}$ & $C, \mathrm{mmol} \mathrm{L}^{-1 a}$ & $k, 10^{-3} \mathrm{~L} \mathrm{~mol}^{-1} \mathrm{~s}^{-1 b}$ & $R^{2 c}$ \\
\hline 1 & 80 & 3.5 & $12.1(5)$ & 0.993 \\
2 & 80 & 6.8 & $12.0(5)$ & 0.9991 \\
3 & 80 & 13.7 & $12.2(5)$ & 0.9989 \\
4 & 80 & 26.9 & $11.3(4)$ & 0.998 \\
\hline \multicolumn{7}{c}{ Initial concentration of $\mathbf{5 .}{ }^{b}$ Calculated second-order reaction rate constants } \\
for the conversion of $\mathbf{5}$ with standard error in parentheses. ${ }^{c}$ Correlation \\
coefficient.
\end{tabular}


Table S2. X-ray Crystallographic Data for $\left[\mathrm{Cp} * \operatorname{Ir}\left(\mu_{2}-\mathrm{NCN}-N, N\right)\right]_{2}(5),\left[\mathrm{Cp} * \operatorname{Ir}(\mathrm{CO})\left(\mu_{2}-\mathrm{NCN}-N, N\right)\left(\mu_{2}-\mathrm{OCNCN}\right) \operatorname{IrCp} *\right](6),\left[\mathrm{Cp} * \operatorname{Ir}(\mathrm{PMe})\left(\mu_{2}-\mathrm{NCN}-N, N\right)_{2} \operatorname{IrCp} *\right]$ (8d), $\left[\left\{\mathrm{Cp} * \operatorname{Ir}\left(\mu_{2}-\mathrm{NCN}-N, N\right)\right\}_{2}(\mu-\mathrm{dppm})\right] \cdot 1.5 \mathrm{C}_{6} \mathrm{H}_{6}\left(\mathbf{9} \cdot 1.5 \mathrm{C}_{6} \mathrm{H}_{6}\right)$, and $\left[(\mathrm{Cp} * \operatorname{Ir})_{2}\{\mathrm{Rh}(\operatorname{cod})\}\left\{\mu_{3}-\mathrm{NCN}-N, N, N\right\}_{2}\right]\left(\mathrm{BPh}_{4}\right)\left(\mathbf{1 0 a B P h}_{4} \cdot \mathrm{CH}_{2} \mathrm{Cl}_{2}\right)$.

\begin{tabular}{|c|c|c|c|c|c|}
\hline & 5 & 6 & 8d & $\mathbf{9} \cdot 1.5 \mathrm{C}_{6} \mathrm{H}_{6}$ & $10 \mathrm{aBPh}_{4} \cdot \mathrm{CH}_{2} \mathrm{Cl}_{2}$ \\
\hline chemical formula & $\mathrm{C}_{22} \mathrm{H}_{30} \mathrm{Ir}_{2} \mathrm{~N}_{4}$ & $\mathrm{C}_{24} \mathrm{H}_{30} \mathrm{Ir}_{2} \mathrm{~N}_{4} \mathrm{O}_{2}$ & $\mathrm{C}_{25} \mathrm{H}_{39} \mathrm{Ir}_{2} \mathrm{~N}_{4} \mathrm{P}$ & $\mathrm{C}_{56} \mathrm{H}_{61} \mathrm{Ir}_{2} \mathrm{~N}_{4} \mathrm{P}_{2}$ & $\mathrm{C}_{55} \mathrm{H}_{64} \mathrm{BCl}_{2} \mathrm{Ir}_{2} \mathrm{Rh}$ \\
\hline formula weight & 734.95 & 790.97 & 811.02 & 1236.51 & 1350.20 \\
\hline crystal color, habit & dark red, needle & dark brown, needle & dark purple, needle & dark red, block & dark red, plate \\
\hline dimensions of crystal & $0.70 \times 0.30 \times 0.20$ & $0.60 \times 0.30 \times 0.10$ & $0.60 \times 0.20 \times 0.05$ & $0.70 \times 0.70 \times 0.40$ & $0.60 \times 0.50 \times 0.10$ \\
\hline diffractometer & Rigaku AFC-7R & Rigaku AFC-7R & Rigaku RAXIS-RAPID & Rigaku RAXIS-RAPID & Rigaku AFC-7S \\
\hline temperature, ${ }^{\circ} \mathrm{C}$ & 21 & 21 & -165 & 21 & 21 \\
\hline crystal system & orthorhombic & triclinic & orthorhombic & triclinic & orthorhombic \\
\hline space group & Pnma & $P \overline{1}$ & Pnma & $P \overline{1}$ & Pccn \\
\hline$a, \AA$ & 21.365(6) & $8.888(5)$ & 19.199(5) & $12.084(4)$ & $12.680(2)$ \\
\hline$b, \AA$ & $15.428(5)$ & $11.704(4)$ & $13.714(5)$ & $12.105(4)$ & $12.734(2)$ \\
\hline$c, \AA$ & $6.697(5)$ & $12.696(4)$ & $9.781(3)$ & $19.525(7)$ & $32.297(4)$ \\
\hline$\alpha$, deg & & 88.75(3) & & 99.37(1) & \\
\hline$\beta$, deg & & $86.05(4)$ & & 91.82(1) & \\
\hline$\gamma$, deg & & 76.30(4) & & $118.88(1)$ & \\
\hline$V, \AA^{3}$ & 2207(1) & $1280.2(9)$ & $2575(1)$ & $2447(1)$ & $5214(1)$ \\
\hline Z & 4 & 2 & 4 & 2 & 4 \\
\hline$\rho_{\text {calcd, }}, \mathrm{g} \mathrm{cm}^{-1}$ & 2.211 & 2.052 & 2.092 & 1.678 & 1.720 \\
\hline$F(000)$ & 1376 & 744 & 1544 & 1218 & 2640 \\
\hline$\mu, \mathrm{cm}^{-1}$ & 120.93 & 104.40 & 104.35 & 55.55 & 55.59 \\
\hline trans. factors range & $0.3752-0.9993$ & $0.4078-0.9967$ & $0.3822-0.8698$ & $0.3663-1.0000$ & $0.3035-0.9962$ \\
\hline $2 \theta$ range, deg & $5<2 \theta<55$ & $5<2 \theta<55$ & $5<2 \theta<55$ & $5<2 \theta<55$ & $5<2 \theta<55$ \\
\hline no. reflections measured & 2908 & 6165 & 24765 & 24110 & 6641 \\
\hline no. unique reflections & 2519 & 5779 & 3028 & 10868 & 5934 \\
\hline$R_{\text {int }}$ & 0.000 & 0.021 & 0.103 & 0.033 & 0.000 \\
\hline no. reflections used $(I>3 \sigma(I))$ & 1731 & 4755 & 2557 & 8788 & 3844 \\
\hline no. parameters refined & 156 & 320 & 184 & 638 & 329 \\
\hline$R(I>3 \sigma(I))^{a}$ & 0.052 & 0.026 & 0.058 & 0.031 & 0.051 \\
\hline$R_{\mathrm{w}}(I>3 \sigma(I))^{b}$ & 0.054 & 0.026 & 0.068 & 0.036 & 0.052 \\
\hline $\operatorname{GOF}(I>3 \sigma(I))^{c}$ & 1.001 & 1.006 & 1.006 & 1.004 & 1.007 \\
\hline
\end{tabular}


Table S3. Positional Parameters and B(eq) for $\left[\mathrm{Cp} * \operatorname{Ir}\left(\mu_{2}-\mathrm{NCN}-N, N\right)\right]_{2}(5)$.

\begin{tabular}{|c|c|c|c|c|c|}
\hline atom & $\mathrm{x}$ & $\mathrm{y}$ & $\mathrm{Z}$ & $B(e q)$ & occupancy \\
\hline $\operatorname{Ir}(1)$ & $0.43198(3)$ & 0.2500 & $0.31218(9)$ & $2.13(1)$ & $1 / 2$ \\
\hline $\operatorname{Ir}(2)$ & $0.30015(3)$ & 0.2500 & $0.3253(1)$ & $2.28(1)$ & $1 / 2$ \\
\hline $\mathrm{N}(1)$ & $0.3668(5)$ & $0.1705(9)$ & $0.429(2)$ & $3.8(3)$ & \\
\hline $\mathrm{N}(2)$ & $0.3667(7)$ & $0.013(1)$ & $0.365(4)$ & $7.7(5)$ & \\
\hline $\mathrm{C}(1)$ & $0.3660(7)$ & $0.087(1)$ & $0.391(3)$ & $5.2(4)$ & \\
\hline $\mathrm{C}(2)$ & $0.4793(7)$ & $0.2032(8)$ & $0.057(2)$ & $3.1(3)$ & \\
\hline C(3) & $0.5140(7)$ & $0.1755(9)$ & $0.228(2)$ & $3.3(3)$ & \\
\hline C(4) & $0.5311(7)$ & 0.2500 & $0.335(2)$ & $2.5(4)$ & $1 / 2$ \\
\hline C(5) & $0.4509(9)$ & $0.148(1)$ & $-0.104(3)$ & $6.0(5)$ & \\
\hline C(6) & $0.524(1)$ & $0.081(1)$ & $0.275(3)$ & $6.9(6)$ & \\
\hline C(7) & $0.569(1)$ & $0.25000(1)$ & $0.514(3)$ & $6.2(7)$ & $1 / 2$ \\
\hline C(8) & $0.2485(6)$ & $0.2026(8)$ & $0.070(2)$ & $2.9(3)$ & \\
\hline $\mathrm{C}(9)$ & $0.2178(6)$ & $0.1756(9)$ & $0.253(2)$ & $3.6(3)$ & \\
\hline $\mathrm{C}(10)$ & $0.1997(8)$ & 0.2500 & $0.364(3)$ & $2.9(4)$ & $1 / 2$ \\
\hline $\mathrm{C}(11)$ & $0.2749(8)$ & $0.144(1)$ & $-0.087(2)$ & 4.3(4) & \\
\hline $\mathrm{C}(12)$ & $0.2074(9)$ & $0.080(1)$ & $0.306(3)$ & $6.0(5)$ & \\
\hline C(13) & $0.163(1)$ & $0.25000(1)$ & $0.555(4)$ & $6.1(7)$ & $1 / 2$ \\
\hline $\mathrm{H}(1)$ & 0.4218 & 0.1100 & -0.0410 & 7.180 & \\
\hline $\mathrm{H}(2)$ & 0.4298 & 0.1814 & -0.2020 & 7.179 & \\
\hline $\mathrm{H}(3)$ & 0.4827 & 0.1142 & -0.1668 & 7.180 & \\
\hline $\mathrm{H}(4)$ & 0.5041 & 0.0692 & 0.3989 & 8.225 & \\
\hline $\mathrm{H}(5)$ & 0.5063 & 0.0455 & 0.1738 & 8.226 & \\
\hline $\mathrm{H}(6)$ & 0.5676 & 0.0685 & 0.2864 & 8.227 & \\
\hline $\mathrm{H}(7)$ & 0.5475 & 0.2192 & 0.6147 & 7.490 & $1 / 2$ \\
\hline $\mathrm{H}(8)$ & 0.6086 & 0.2227 & 0.4899 & 7.489 & $1 / 2$ \\
\hline $\mathrm{H}(9)$ & 0.5762 & 0.3080 & 0.5561 & 7.490 & $1 / 2$ \\
\hline $\mathrm{H}(10)$ & 0.2423 & 0.1093 & -0.1403 & 5.190 & \\
\hline $\mathrm{H}(11)$ & 0.2932 & 0.1774 & -0.1918 & 5.190 & \\
\hline $\mathrm{H}(12)$ & 0.3059 & 0.1082 & -0.0288 & 5.191 & \\
\hline $\mathrm{H}(13)$ & 0.1639 & 0.0697 & 0.3234 & 7.247 & \\
\hline $\mathrm{H}(14)$ & 0.2233 & 0.0430 & 0.2051 & 7.246 & \\
\hline $\mathrm{H}(15)$ & 0.2286 & 0.0692 & 0.4282 & 7.247 & \\
\hline $\mathrm{H}(16)$ & 0.1571 & 0.3080 & 0.5995 & 7.299 & $1 / 2$ \\
\hline $\mathrm{H}(17)$ & 0.1230 & 0.2236 & 0.5356 & 7.298 & $1 / 2$ \\
\hline $\mathrm{H}(18)$ & 0.1855 & 0.2184 & 0.6527 & 7.298 & $1 / 2$ \\
\hline
\end{tabular}


Table S4. Anisotropic Displacement Parameters for $\left[\mathrm{Cp} * \operatorname{Ir}\left(\mu_{2}-\mathrm{NCN}-N, N\right)\right]_{2}$ (5).

\begin{tabular}{llllccc}
\hline atom & $\mathrm{U} 11$ & $\mathrm{U} 22$ & $\mathrm{U} 33$ & $\mathrm{U} 12$ & $\mathrm{U} 13$ & $\mathrm{U} 23$ \\
\hline $\mathrm{Ir}(1)$ & $0.0159(4)$ & $0.0426(4)$ & $0.0224(3)$ & 0.0000 & $-0.0008(3)$ & 0.0000 \\
$\mathrm{Ir}(2)$ & $0.0159(4)$ & $0.0470(4)$ & $0.0237(3)$ & 0.0000 & $-0.0049(3)$ & 0.0000 \\
$\mathrm{~N}(1)$ & $0.014(5)$ & $0.085(9)$ & $0.043(7)$ & $0.007(6)$ & $-0.002(5)$ & $0.029(6)$ \\
$\mathrm{N}(2)$ & $0.06(1)$ & $0.07(1)$ & $0.17(2)$ & $-0.006(8)$ & $-0.01(1)$ & $0.05(1)$ \\
$\mathrm{C}(1)$ & $0.023(7)$ & $0.07(1)$ & $0.11(1)$ & $0.001(8)$ & $-0.003(9)$ & $0.05(1)$ \\
$\mathrm{C}(2)$ & $0.041(9)$ & $0.039(7)$ & $0.038(7)$ & $-0.008(6)$ & $0.008(7)$ & $-0.006(6)$ \\
$\mathrm{C}(3)$ & $0.032(7)$ & $0.050(8)$ & $0.043(8)$ & $0.013(6)$ & $0.012(7)$ & $0.016(6)$ \\
$\mathrm{C}(4)$ & $0.003(7)$ & $0.08(1)$ & $0.017(8)$ & 0.0000 & $0.006(7)$ & 0.0000 \\
$\mathrm{C}(5)$ & $0.08(1)$ & $0.10(1)$ & $0.06(1)$ & $-0.02(1)$ & $0.02(1)$ & $-0.03(1)$ \\
$\mathrm{C}(6)$ & $0.08(2)$ & $0.07(1)$ & $0.11(2)$ & $0.04(1)$ & $0.03(1)$ & $0.04(1)$ \\
$\mathrm{C}(7)$ & $0.01(1)$ & $0.18(3)$ & $0.04(1)$ & 0.0000 & $0.00(1)$ & 0.0000 \\
$\mathrm{C}(8)$ & $0.029(7)$ & $0.046(7)$ & $0.033(7)$ & $-0.005(6)$ & $-0.017(6)$ & $-0.005(6)$ \\
$\mathrm{C}(9)$ & $0.026(7)$ & $0.052(9)$ & $0.058(9)$ & $-0.013(6)$ & $-0.009(7)$ & $0.017(7)$ \\
$\mathrm{C}(10)$ & $0.006(7)$ & $0.07(1)$ & $0.033(9)$ & 0.0000 & $-0.005(7)$ & 0.0000 \\
$\mathrm{C}(11)$ & $0.06(1)$ & $0.06(1)$ & $0.046(8)$ & $0.016(8)$ & $-0.009(8)$ & $-0.023(7)$ \\
$\mathrm{C}(12)$ & $0.06(1)$ & $0.06(1)$ & $0.11(2)$ & $-0.033(9)$ & $-0.03(1)$ & $0.03(1)$ \\
$\mathrm{C}(13)$ & $0.02(1)$ & $0.16(3)$ & $0.06(1)$ & 0.0000 & $0.00(1)$ & 0.0000 \\
\hline
\end{tabular}

Table S5. Intramolecular Distances Involving the Nonhydrogen Atoms for $\left[\mathrm{Cp} * \operatorname{Ir}\left(\mu_{2}-\mathrm{NCN}-N, N\right)\right]_{2}(5)$.

\begin{tabular}{lll|lll}
\hline atom & atom & distance & atom & atom & distance \\
\hline $\operatorname{Ir}(1)$ & $\operatorname{Ir}(2)$ & $2.8179(9)$ & $\operatorname{Ir}(1)$ & $\mathrm{N}(1)$ & $2.01(1)$ \\
$\operatorname{Ir}(1)$ & $\mathrm{N}(1)^{1)}$ & $2.01(1)$ & $\operatorname{Ir}(1)$ & $\mathrm{C}(2)$ & $2.11(1)$ \\
$\operatorname{Ir}(1)$ & $\mathrm{C}(2)^{1)}$ & $2.11(1)$ & $\operatorname{Ir}(1)$ & $\mathrm{C}(3)$ & $2.17(1)$ \\
$\operatorname{Ir}(1)$ & $\mathrm{C}(3)^{1)}$ & $2.17(1)$ & $\operatorname{Ir}(1)$ & $\mathrm{C}(4)$ & $2.12(1)$ \\
$\operatorname{Ir}(2)$ & $\mathrm{N}(1)$ & $2.00(1)$ & $\operatorname{Ir}(2)$ & $\mathrm{N}(1)^{1)}$ & $2.00(1)$ \\
$\operatorname{Ir}(2)$ & $\mathrm{C}(8)$ & $2.16(1)$ & $\operatorname{Ir}(2)$ & $\mathrm{C}(8)^{1)}$ & $2.16(1)$ \\
$\operatorname{Ir}(2)$ & $\mathrm{C}(9)$ & $2.16(1)$ & $\operatorname{Ir}(2)$ & $\mathrm{C}(9)^{1)}$ & $2.16(1)$ \\
$\operatorname{Ir}(2)$ & $\mathrm{C}(10)$ & $2.16(2)$ & $\mathrm{N}(1)$ & $\mathrm{C}(1)$ & $1.32(2)$ \\
$\mathrm{N}(2)$ & $\mathrm{C}(1)$ & $1.15(3)$ & $\mathrm{C}(2)$ & $\mathrm{C}(2)^{1)}$ & $1.44(2)$ \\
$\mathrm{C}(2)$ & $\mathrm{C}(3)$ & $1.43(2)$ & $\mathrm{C}(2)$ & $\mathrm{C}(5)$ & $1.51(2)$ \\
$\mathrm{C}(3)$ & $\mathrm{C}(4)$ & $1.40(2)$ & $\mathrm{C}(3)$ & $\mathrm{C}(6)$ & $1.51(2)$ \\
$\mathrm{C}(4)$ & $\mathrm{C}(3)^{1)}$ & $1.40(2)$ & $\mathrm{C}(4)$ & $\mathrm{C}(7)$ & $1.45(3)$ \\
$\mathrm{C}(8)$ & $\mathrm{C}(8)^{1)}$ & $1.46(2)$ & $\mathrm{C}(8)$ & $\mathrm{C}(9)$ & $1.45(2)$ \\
$\mathrm{C}(8)$ & $\mathrm{C}(11)$ & $1.50(2)$ & $\mathrm{C}(9)$ & $\mathrm{C}(10)$ & $1.42(2)$ \\
$\mathrm{C}(9)$ & $\mathrm{C}(12)$ & $1.53(2)$ & $\mathrm{C}(10)$ & $\mathrm{C}(9)^{1)}$ & $1.42(2)$ \\
$\mathrm{C}(10)$ & $\mathrm{C}(13)$ & $1.50(3)$ & & & \\
\hline
\end{tabular}

Distances are in angstroms. Symmetry operations, 1$):-\mathrm{X},-\mathrm{Y}+1 / 2, \mathrm{Z}$.

Table S6. Intramolecular Bond Angles Involving the Nonhydrogen Atoms for $\left[\mathrm{Cp} * \operatorname{Ir}\left(\mu_{2}-\mathrm{NCN}-N, N\right)\right]_{2}(5)$.

\begin{tabular}{llll|llll}
\hline atom & atom & atom & angle & atom & atom & atom & \multicolumn{1}{c}{ angle } \\
\hline $\operatorname{Ir}(1)$ & $\operatorname{Ir}(2)$ & $\mathrm{N}(1)$ & $45.6(4)$ & $\mathrm{N}(1)$ & $\operatorname{Ir}(1)$ & $\operatorname{Ir}(2)$ & $45.3(4)$ \\
$\operatorname{Ir}(1)$ & $\operatorname{Ir}(2)$ & $\mathrm{N}(1)^{1)}$ & $45.6(4)$ & $\left.\mathrm{N}(1)^{1}\right)$ & $\operatorname{Ir}(1)$ & $\operatorname{Ir}(2)$ & $45.3(3)$ \\
$\mathrm{C}(2)$ & $\operatorname{Ir}(1)$ & $\operatorname{Ir}(2)$ & $120.2(4)$ & $\mathrm{C}(2)^{1)}$ & $\operatorname{Ir}(1)$ & $\operatorname{Ir}(2)$ & $120.2(4)$ \\
$\mathrm{C}(3)$ & $\operatorname{Ir}(1)$ & $\operatorname{Ir}(2)$ & $144.6(4)$ & $\mathrm{C}(3)^{1)}$ & $\operatorname{Ir}(1)$ & $\operatorname{Ir}(2)$ & $144.6(4)$ \\
$\mathrm{C}(4)$ & $\operatorname{Ir}(1)$ & $\operatorname{Ir}(2)$ & $174.1(4)$ & $\operatorname{Ir}(1)$ & $\operatorname{Ir}(2)$ & $\mathrm{C}(8)$ & $119.0(4)$ \\
$\operatorname{Ir}(1)$ & $\operatorname{Ir}(2)$ & $\mathrm{C}(8)^{1)}$ & $119.0(4)$ & $\operatorname{Ir}(1)$ & $\operatorname{Ir}(2)$ & $\mathrm{C}(9)$ & $144.0(4)$ \\
$\operatorname{Ir}(1)$ & $\operatorname{Ir}(2)$ & $\mathrm{C}(9)^{1)}$ & $144.0(4)$ & $\operatorname{Ir}(1)$ & $\operatorname{Ir}(2)$ & $\mathrm{C}(10)$ & $174.9(5)$ \\
$\operatorname{Ir}(1)$ & $\mathrm{N}(1)$ & $\operatorname{Ir}(2)$ & $89.1(5)$ & $\mathrm{N}(1)^{1)}$ & $\operatorname{Ir}(1)$ & $\mathrm{N}(1)$ & $75.1(5)$ \\
$\operatorname{Ir}(1)$ & $\mathrm{N}(1)$ & $\mathrm{C}(1)$ & $122(1)$ & $\mathrm{C}(2)$ & $\operatorname{Ir}(1)$ & $\mathrm{N}(1)$ & $115.9(5)$ \\
$\mathrm{C}(2)^{1)}$ & $\operatorname{Ir}(1)$ & $\mathrm{N}(1)$ & $148.6(5)$ & $\mathrm{C}(3)$ & $\operatorname{Ir}(1)$ & $\mathrm{N}(1)$ & $109.7(5)$ \\
$\mathrm{C}(3)^{1)}$ & $\operatorname{Ir}(1)$ & $\mathrm{N}(1)$ & $169.1(5)$ & $\mathrm{C}(4)$ & $\operatorname{Ir}(1)$ & $\mathrm{N}(1)$ & $131.4(4)$ \\
$\operatorname{Ir}(1)$ & $\mathrm{N}(1)^{1)}$ & $\operatorname{Ir}(2)$ & $89.1(5)$ & $\operatorname{Ir}(1)$ & $\mathrm{N}(1)^{1)}$ & $\mathrm{C}(1)^{1)}$ & $122(1)$ \\
$\mathrm{C}(2)$ & $\operatorname{Ir}(1)$ & $\mathrm{N}(1)^{1)}$ & $148.6(5)$ & $\mathrm{C}(2)^{1)}$ & $\operatorname{Ir}(1)$ & $\mathrm{N}(1)^{1)}$ & $115.9(5)$
\end{tabular}




\begin{tabular}{|c|c|c|c|c|c|c|c|}
\hline C(3) & $\operatorname{Ir}(1)$ & $\mathrm{N}(1)^{1)}$ & 169.1(5) & $C(3)^{1)}$ & $\operatorname{Ir}(1)$ & $\mathrm{N}(1)^{1)}$ & 109.7(5) \\
\hline $\mathrm{C}(4)$ & $\operatorname{Ir}(1)$ & $\mathrm{N}(1)^{1)}$ & $131.4(4)$ & $\operatorname{Ir}(1)$ & $C(2)$ & $\mathrm{C}(2)^{1)}$ & $70.0(8)$ \\
\hline$C(2)^{1)}$ & $\operatorname{Ir}(1)$ & $\mathrm{C}(2)$ & $40.0(5)$ & $\operatorname{Ir}(1)$ & $\mathrm{C}(2)$ & C(3) & $72.7(8)$ \\
\hline C(3) & $\operatorname{Ir}(1)$ & $\mathrm{C}(2)$ & $38.9(5)$ & $C(3)^{1)}$ & $\operatorname{Ir}(1)$ & $\mathrm{C}(2)$ & $65.4(5)$ \\
\hline C(4) & $\operatorname{Ir}(1)$ & $C(2)$ & $65.2(6)$ & $\operatorname{Ir}(1)$ & $\mathrm{C}(2)$ & C(5) & $125(1)$ \\
\hline $\operatorname{Ir}(1)$ & $C(2)^{1)}$ & $\mathrm{C}(2)$ & $70.0(8)$ & $\mathrm{C}(3)$ & $\operatorname{Ir}(1)$ & $C(2)^{1)}$ & 65.4(5) \\
\hline $\operatorname{Ir}(1)$ & $C(2)^{1)}$ & $C(3)^{1)}$ & $72.7(8)$ & $C(3)^{1)}$ & $\operatorname{Ir}(1)$ & $C(2)^{1)}$ & $38.9(5)$ \\
\hline C(4) & $\operatorname{Ir}(1)$ & $C(2)^{1)}$ & $65.2(6)$ & $\operatorname{Ir}(1)$ & $C(2)^{1)}$ & $C(5)^{1)}$ & $125(1)$ \\
\hline $\operatorname{Ir}(1)$ & $\mathrm{C}(3)$ & $\mathrm{C}(2)$ & $68.4(8)$ & $C(3)^{1)}$ & $\operatorname{Ir}(1)$ & C(3) & 63.9(5) \\
\hline $\operatorname{Ir}(1)$ & $\mathrm{C}(3)$ & $\mathrm{C}(4)$ & 69.1(8) & $C(4)$ & $\operatorname{Ir}(1)$ & C(3) & $38.1(4)$ \\
\hline $\operatorname{Ir}(1)$ & C(3) & $\mathrm{C}(6)$ & $125(1)$ & $\operatorname{Ir}(1)$ & $C(3)^{1)}$ & $C(2)^{1)}$ & 68.4(8) \\
\hline $\operatorname{Ir}(1)$ & $C(3)^{1)}$ & $C(4)$ & 69.1(8) & $\mathrm{C}(4)$ & $\operatorname{Ir}(1)$ & $\mathrm{C}(3)^{1)}$ & $38.1(4)$ \\
\hline $\operatorname{Ir}(1)$ & $C(3)^{1)}$ & $C(6)^{1)}$ & $125(1)$ & $\operatorname{Ir}(1)$ & C(4) & C(3) & 72.7(8) \\
\hline $\operatorname{Ir}(1)$ & $C(4)$ & $C(3)^{1)}$ & $72.7(8)$ & $\operatorname{Ir}(1)$ & $\mathrm{C}(4)$ & $\mathrm{C}(7)$ & $128(1)$ \\
\hline $\mathrm{N}(1)^{1)}$ & $\operatorname{Ir}(2)$ & $\mathrm{N}(1)$ & $75.5(5)$ & $\operatorname{Ir}(2)$ & $\mathrm{N}(1)$ & $\mathrm{C}(1)$ & 121(1) \\
\hline $\mathrm{C}(8)$ & $\operatorname{Ir}(2)$ & $\mathrm{N}(1)$ & $115.4(5)$ & $\mathrm{C}(8)^{1)}$ & $\operatorname{Ir}(2)$ & $\mathrm{N}(1)$ & $147.5(5)$ \\
\hline C(9) & $\operatorname{Ir}(2)$ & $\mathrm{N}(1)$ & $109.4(5)$ & $\mathrm{C}(9)^{1)}$ & $\operatorname{Ir}(2)$ & $\mathrm{N}(1)$ & 169.6(5) \\
\hline C(10) & $\operatorname{Ir}(2)$ & $\mathrm{N}(1)$ & $131.6(4)$ & $\operatorname{Ir}(2)$ & $\mathrm{N}(1)^{1)}$ & $\mathrm{C}(1)^{1)}$ & $121(1)$ \\
\hline $\mathrm{C}(8)$ & $\operatorname{Ir}(2)$ & $\mathrm{N}(1)^{1)}$ & $147.5(5)$ & $C(8)^{1)}$ & $\operatorname{Ir}(2)$ & $\mathrm{N}(1)^{1)}$ & $115.4(5)$ \\
\hline $\mathrm{C}(9)$ & $\operatorname{Ir}(2)$ & $\mathrm{N}(1)^{1)}$ & $169.6(5)$ & $\mathrm{C}(9)^{1)}$ & $\operatorname{Ir}(2)$ & $\mathrm{N}(1)^{1)}$ & $109.4(5)$ \\
\hline $\mathrm{C}(10)$ & $\operatorname{Ir}(2)$ & $\mathrm{N}(1)^{1)}$ & $131.6(4)$ & $\operatorname{Ir}(2)$ & $\mathrm{C}(8)$ & $C(8)^{1)}$ & $70.2(7)$ \\
\hline$\left.C(8)^{1}\right)$ & $\operatorname{Ir}(2)$ & C(8) & 39.5(5) & $\operatorname{Ir}(2)$ & C(8) & $\mathrm{C}(9)$ & $70.0(8)$ \\
\hline C(9) & $\operatorname{Ir}(2)$ & $\mathrm{C}(8)$ & 39.3(5) & $C(9)^{1)}$ & $\operatorname{Ir}(2)$ & C(8) & 65.6(5) \\
\hline $\mathrm{C}(10)$ & $\operatorname{Ir}(2)$ & $\mathrm{C}(8)$ & $65.7(6)$ & $\operatorname{Ir}(2)$ & C(8) & $\mathrm{C}(11)$ & $124(1)$ \\
\hline $\operatorname{Ir}(2)$ & $C(8)^{1)}$ & $\mathrm{C}(8)$ & $70.2(7)$ & $\mathrm{C}(9)$ & $\operatorname{Ir}(2)$ & $C(8)^{1)}$ & 65.6(5) \\
\hline $\operatorname{Ir}(2)$ & $C(8)^{1)}$ & $C(9)^{1)}$ & $70.0(8)$ & $\mathrm{C}(9)^{1)}$ & $\operatorname{Ir}(2)$ & $C(8)^{1)}$ & $39.3(5)$ \\
\hline $\mathrm{C}(10)$ & $\operatorname{Ir}(2)$ & $C(8)^{1)}$ & $65.7(6)$ & $\operatorname{Ir}(2)$ & $\mathrm{C}(8)^{1)}$ & $C(11)^{1)}$ & $124(1)$ \\
\hline $\operatorname{Ir}(2)$ & C(9) & C(8) & $70.6(8)$ & $\mathrm{C}(9)^{1)}$ & $\operatorname{Ir}(2)$ & $\mathrm{C}(9)$ & 64.3(5) \\
\hline $\operatorname{Ir}(2)$ & $\mathrm{C}(9)$ & $C(10)$ & 71.0(9) & $C(10)$ & $\operatorname{Ir}(2)$ & $\mathrm{C}(9)$ & 38.4(5) \\
\hline $\operatorname{Ir}(2)$ & $\mathrm{C}(9)$ & $C(12)$ & $125(1)$ & $\operatorname{Ir}(2)$ & $\mathrm{C}(9)^{1)}$ & $C(8)^{1)}$ & $70.6(8)$ \\
\hline $\operatorname{Ir}(2)$ & $C(9)^{1)}$ & $\mathrm{C}(10)$ & $71.0(9)$ & $\mathrm{C}(10)$ & $\operatorname{Ir}(2)$ & $C(9)^{1)}$ & $38.4(5)$ \\
\hline $\operatorname{Ir}(2)$ & $C(9)^{1)}$ & $\mathrm{C}(12)^{1)}$ & $125(1)$ & $\operatorname{Ir}(2)$ & $C(10)$ & $\mathrm{C}(9)$ & 70.6(9) \\
\hline $\operatorname{Ir}(2)$ & $C(10)$ & $\mathrm{C}(9)^{1)}$ & $70.6(9)$ & $\operatorname{Ir}(2)$ & $C(10)$ & $C(13)$ & $128(1)$ \\
\hline $\mathrm{N}(1)$ & $\mathrm{C}(1)$ & $\mathrm{N}(2)$ & $177(1)$ & C(3) & $\mathrm{C}(2)$ & $C(2)^{1)}$ & 107(1) \\
\hline C(2) & $C(2)^{1)}$ & $C(3)^{1)}$ & 107(1) & C(5) & $\mathrm{C}(2)$ & $C(2)^{1)}$ & $124(1)$ \\
\hline C(2) & $C(2)^{1)}$ & $C(5)^{1)}$ & 124(1) & $\mathrm{C}(2)$ & $\mathrm{C}(3)$ & $\mathrm{C}(4)$ & 107(1) \\
\hline C(5) & $\mathrm{C}(2)$ & C(3) & 127(1) & $\mathrm{C}(2)$ & $\mathrm{C}(3)$ & C(6) & 122(1) \\
\hline C(3) & $\mathrm{C}(4)$ & $C(3)^{1)}$ & $109(1)$ & $\mathrm{C}(6)$ & C(3) & $C(4)$ & $130(1)$ \\
\hline C(3) & $C(4)$ & $\mathrm{C}(7)$ & $124.7(8)$ & $\mathrm{C}(4)$ & $C(3)^{1)}$ & $C(2)^{1)}$ & 107(1) \\
\hline C(4) & $C(3)^{1)}$ & $C(6)^{1)}$ & $130(1)$ & $\mathrm{C}(7)$ & $\mathrm{C}(4)$ & $C(3)^{1)}$ & $124.7(8)$ \\
\hline C(9) & $\mathrm{C}(8)$ & $C(8)^{1)}$ & 106(1) & $\mathrm{C}(8)$ & $\mathrm{C}(8)^{1)}$ & $\mathrm{C}(9)^{1)}$ & $106(1)$ \\
\hline $\mathrm{C}(11)$ & $\mathrm{C}(8)$ & $C(8)^{1)}$ & 126(1) & $\mathrm{C}(8)$ & $C(8)^{1)}$ & $C(11)^{1)}$ & $126(1)$ \\
\hline $\mathrm{C}(8)$ & C(9) & C(10) & 109(1) & C(11) & C(8) & C(9) & $126(1)$ \\
\hline C(8) & $\mathrm{C}(9)$ & $C(12)$ & 122(1) & $\mathrm{C}(9)$ & $C(10)$ & $C(9)^{1)}$ & $107(1)$ \\
\hline $\mathrm{C}(12)$ & C(9) & C(10) & $128(1)$ & C(9) & $\mathrm{C}(10)$ & C(13) & $126.0(8)$ \\
\hline $\mathrm{C}(10)$ & $\mathrm{C}(9)^{1)}$ & $C(8)^{1)}$ & 109(1) & $\mathrm{C}(10)$ & $C(9)^{1)}$ & $\mathrm{C}(12)^{1)}$ & $128(1)$ \\
\hline $\mathrm{C}(13)$ & $\mathrm{C}(10)$ & $C(9)^{1)}$ & $126.0(8)$ & & & & \\
\hline
\end{tabular}

Angles are in degrees. Symmetry operations, 1$):-\mathrm{X},-\mathrm{Y}+1 / 2$, Z . 
Table S7. Positional Parameters and B(eq) for $\left[\mathrm{Cp} * \operatorname{Ir}(\mathrm{CO})\left(\mu_{2}-\mathrm{NCN}-N, N\right)\left(\mu_{2}-\mathrm{OCNCN}\right) \operatorname{IrCp}{ }^{*}\right](6)$.

\begin{tabular}{|c|c|c|c|c|}
\hline atom & $\mathrm{X}$ & $\mathrm{y}$ & $\mathrm{Z}$ & $\mathrm{B}(\mathrm{eq})$ \\
\hline $\operatorname{Ir}(1)$ & $0.75251(3)$ & $0.64738(2)$ & $0.76861(2)$ & $3.547(6)$ \\
\hline $\operatorname{Ir}(2)$ & $0.66854(2)$ & $0.96071(2)$ & $0.77909(1)$ & $2.503(4)$ \\
\hline $\mathrm{O}(1)$ & $0.4675(5)$ & $0.7028(5)$ & $0.6589(5)$ & $7.1(1)$ \\
\hline $\mathrm{O}(2)$ & $0.550(1)$ & $0.5936(7)$ & $0.9517(7)$ & $14.6(3)$ \\
\hline $\mathrm{N}(1)$ & $0.5312(5)$ & $0.8681(4)$ & $0.7117(4)$ & $3.5(1)$ \\
\hline $\mathrm{N}(2)$ & $0.2909(7)$ & $0.9836(6)$ & $0.6348(5)$ & $6.6(2)$ \\
\hline $\mathrm{N}(3)$ & $0.7620(5)$ & $0.8053(4)$ & $0.8366(3)$ & $3.22(9)$ \\
\hline $\mathrm{N}(4)$ & $0.9530(7)$ & $0.7755(5)$ & $0.9740(4)$ & $5.0(1)$ \\
\hline $\mathrm{C}(1)$ & $0.5558(7)$ & $0.7451(6)$ & $0.7048(5)$ & 4.4(1) \\
\hline $\mathrm{C}(2)$ & $0.4020(7)$ & $0.9287(6)$ & $0.6686(5)$ & $4.4(1)$ \\
\hline $\mathrm{C}(3)$ & $0.8637(7)$ & $0.7898(5)$ & $0.9090(4)$ & $3.5(1)$ \\
\hline$C(4)$ & $0.630(1)$ & $0.6151(7)$ & $0.8821(7)$ & $7.6(3)$ \\
\hline $\mathrm{C}(5)$ & $0.8825(7)$ & $0.6176(5)$ & $0.6097(4)$ & $4.2(1)$ \\
\hline$C(6)$ & 0.9921(7) & $0.6261(5)$ & $0.6832(5)$ & $4.2(1)$ \\
\hline$C(7)$ & $1.0015(8)$ & $0.5352(6)$ & $0.7591(6)$ & $5.2(2)$ \\
\hline $\mathrm{C}(8)$ & $0.896(1)$ & $0.4674(5)$ & $0.7326(6)$ & $5.6(2)$ \\
\hline C(9) & $0.8193(8)$ & $0.5174(6)$ & $0.6400(5)$ & $5.1(2)$ \\
\hline$C(10)$ & $0.845(1)$ & $0.6928(8)$ & $0.5139(6)$ & $7.5(2)$ \\
\hline $\mathrm{C}(11)$ & $1.0834(8)$ & $0.7184(7)$ & $0.6812(7)$ & $6.7(2)$ \\
\hline$C(12)$ & $1.114(1)$ & $0.5056(8)$ & $0.8448(7)$ & $8.4(3)$ \\
\hline $\mathrm{C}(13)$ & $0.872(2)$ & $0.3563(7)$ & $0.7861(8)$ & 10.2(3) \\
\hline $\mathrm{C}(14)$ & $0.711(1)$ & $0.4690(8)$ & $0.5789(7)$ & $8.1(3)$ \\
\hline C(15) & $0.7708(6)$ & $1.0918(5)$ & $0.7028(4)$ & $3.2(1)$ \\
\hline$C(16)$ & $0.8218(6)$ & $1.0733(4)$ & $0.8096(4)$ & $3.0(1)$ \\
\hline$C(17)$ & $0.6868(6)$ & $1.1019(4)$ & $0.8792(4)$ & 3.2(1) \\
\hline C(18) & $0.5512(6)$ & $1.1408(5)$ & $0.8164(4)$ & 3.6(1) \\
\hline C(19) & $0.6068(6)$ & $1.1381(5)$ & $0.7080(4)$ & 3.6(1) \\
\hline C(20) & $0.8761(8)$ & $1.0735(6)$ & $0.6044(4)$ & 4.6(1) \\
\hline$C(21)$ & $0.9854(6)$ & $1.0350(5)$ & $0.8390(5)$ & $3.8(1)$ \\
\hline $\mathrm{C}(22)$ & $0.6838(8)$ & $1.0992(6)$ & $0.9975(4)$ & $4.8(2)$ \\
\hline C(23) & $0.3867(8)$ & $1.1828(7)$ & $0.8595(6)$ & $6.0(2)$ \\
\hline C(24) & $0.5117(8)$ & $1.1785(6)$ & $0.6159(5)$ & $5.6(2)$ \\
\hline $\mathrm{H}(1)$ & 0.8071 & 0.7733 & 0.5321 & 8.258 \\
\hline $\mathrm{H}(2)$ & 0.9345 & 0.6844 & 0.4666 & 8.260 \\
\hline $\mathrm{H}(3)$ & 0.7671 & 0.6659 & 0.4809 & 8.258 \\
\hline $\mathrm{H}(4)$ & 1.1474 & 0.7046 & 0.7393 & 8.077 \\
\hline $\mathrm{H}(5)$ & 1.1467 & 0.7123 & 0.6172 & 8.074 \\
\hline $\mathrm{H}(6)$ & 1.0171 & 0.7950 & 0.6865 & 8.074 \\
\hline $\mathrm{H}(7)$ & 1.0630 & 0.4980 & 0.9120 & 9.480 \\
\hline $\mathrm{H}(8)$ & 1.1884 & 0.4345 & 0.8281 & 9.477 \\
\hline $\mathrm{H}(9)$ & 1.1647 & 0.5684 & 0.8465 & 9.477 \\
\hline $\mathrm{H}(10)$ & 0.8265 & 0.3794 & 0.8546 & 12.144 \\
\hline $\mathrm{H}(11)$ & 0.8015 & 0.3259 & 0.7483 & 12.145 \\
\hline $\mathrm{H}(12)$ & 0.9651 & 0.2975 & 0.7920 & 12.144 \\
\hline $\mathrm{H}(13)$ & 0.6194 & 0.5294 & 0.5740 & 9.913 \\
\hline $\mathrm{H}(14)$ & 0.7560 & 0.4471 & 0.5100 & 9.913 \\
\hline $\mathrm{H}(15)$ & 0.6869 & 0.4027 & 0.6142 & 9.915 \\
\hline $\mathrm{H}(16)$ & 0.9811 & 1.0426 & 0.6197 & 5.672 \\
\hline $\mathrm{H}(17)$ & 0.8436 & 1.0198 & 0.5611 & 5.674 \\
\hline $\mathrm{H}(18)$ & 0.8669 & 1.1466 & 0.5684 & 5.674 \\
\hline $\mathrm{H}(19)$ & 0.9987 & 0.9655 & 0.8808 & 4.746 \\
\hline $\mathrm{H}(20)$ & 1.0506 & 1.0198 & 0.7760 & 4.743 \\
\hline $\mathrm{H}(21)$ & 1.0120 & 1.0953 & 0.8776 & 4.745 \\
\hline $\mathrm{H}(22)$ & 0.5799 & 1.1242 & 1.0262 & 5.740 \\
\hline $\mathrm{H}(23)$ & 0.7263 & 1.0209 & 1.0198 & 5.744 \\
\hline
\end{tabular}




\begin{tabular}{lllll}
$\mathrm{H}(24)$ & 0.7438 & 1.1496 & 1.0213 & 5.745 \\
$\mathrm{H}(25)$ & 0.3478 & 1.2625 & 0.8395 & 6.812 \\
$\mathrm{H}(26)$ & 0.3266 & 1.1353 & 0.8308 & 6.812 \\
$\mathrm{H}(27)$ & 0.3805 & 1.1763 & 0.9343 & 6.809 \\
$\mathrm{H}(28)$ & 0.5468 & 1.2403 & 0.5800 & 6.733 \\
$\mathrm{H}(29)$ & 0.5232 & 1.1141 & 0.5693 & 6.732 \\
$\mathrm{H}(30)$ & 0.4054 & 1.2055 & 0.6390 & 6.733 \\
\hline
\end{tabular}

Table S8. Anisotropic Displacement Parameters for $\left[\mathrm{Cp} * \operatorname{Ir}(\mathrm{CO})\left(\mu_{2}-\mathrm{NCN}-N, N\right)\left(\mu_{2}-\mathrm{OCNCN}\right) \operatorname{IrCp} *\right](6)$.

\begin{tabular}{|c|c|c|c|c|c|c|}
\hline atom & U11 & U22 & U33 & U12 & U13 & U23 \\
\hline $\operatorname{Ir}(1)$ & $0.0592(1)$ & $0.0311(1)$ & $0.0477(1)$ & $-0.0170(1)$ & $-0.0041(1)$ & $0.00118(9)$ \\
\hline $\operatorname{Ir}(2)$ & $0.0309(1)$ & $0.0316(1)$ & $0.0324(1)$ & $-0.00606(8)$ & $-0.00482(7)$ & $0.00008(7)$ \\
\hline $\mathrm{O}(1)$ & $0.057(3)$ & $0.075(3)$ & $0.145(5)$ & $-0.025(3)$ & $-0.027(3)$ & $-0.034(3)$ \\
\hline $\mathrm{O}(2)$ & $0.28(1)$ & $0.125(6)$ & $0.157(7)$ & $-0.105(7)$ & 0.113(8) & $0.009(5)$ \\
\hline $\mathrm{N}(1)$ & $0.034(2)$ & $0.052(3)$ & $0.050(3)$ & $-0.012(2)$ & $-0.008(2)$ & $-0.010(2)$ \\
\hline $\mathrm{N}(2)$ & $0.050(3)$ & $0.104(5)$ & $0.092(4)$ & $0.000(3)$ & $-0.034(3)$ & $-0.018(4)$ \\
\hline $\mathrm{N}(3)$ & $0.052(3)$ & $0.030(2)$ & $0.040(2)$ & $-0.010(2)$ & $-0.005(2)$ & $0.001(2)$ \\
\hline $\mathrm{N}(4)$ & $0.088(4)$ & $0.045(3)$ & $0.057(3)$ & $-0.008(3)$ & $-0.028(3)$ & $0.001(2)$ \\
\hline $\mathrm{C}(1)$ & $0.045(3)$ & $0.059(4)$ & $0.065(4)$ & $-0.022(3)$ & $0.000(3)$ & $-0.012(3)$ \\
\hline $\mathrm{C}(2)$ & $0.043(3)$ & $0.068(4)$ & $0.058(3)$ & $-0.017(3)$ & $-0.005(3)$ & $-0.020(3)$ \\
\hline C(3) & $0.056(3)$ & $0.034(3)$ & $0.040(3)$ & $-0.007(2)$ & $-0.007(2)$ & $0.001(2)$ \\
\hline C(4) & $0.131(8)$ & $0.060(5)$ & $0.105(6)$ & $-0.047(5)$ & $0.025(6)$ & $0.005(4)$ \\
\hline C(5) & $0.055(3)$ & $0.051(4)$ & $0.046(3)$ & $0.005(3)$ & $-0.006(3)$ & $-0.001(3)$ \\
\hline$C(6)$ & $0.042(3)$ & $0.048(3)$ & $0.064(4)$ & $0.005(3)$ & $-0.002(3)$ & $-0.009(3)$ \\
\hline C(7) & $0.077(5)$ & $0.040(3)$ & $0.073(4)$ & $0.014(3)$ & $-0.029(4)$ & $-0.012(3)$ \\
\hline C(8) & $0.107(6)$ & $0.030(3)$ & $0.072(4)$ & $-0.007(3)$ & $-0.020(4)$ & $-0.000(3)$ \\
\hline C(9) & $0.070(4)$ & $0.056(4)$ & $0.066(4)$ & $-0.013(3)$ & $-0.007(3)$ & $-0.021(3)$ \\
\hline$C(10)$ & $0.080(5)$ & $0.120(7)$ & $0.062(4)$ & $0.022(5)$ & $-0.001(4)$ & $0.025(5)$ \\
\hline $\mathrm{C}(11)$ & $0.055(4)$ & $0.086(6)$ & $0.115(7)$ & $-0.022(4)$ & $0.012(4)$ & $-0.028(5)$ \\
\hline $\mathrm{C}(12)$ & $0.126(8)$ & $0.080(6)$ & $0.094(6)$ & $0.031(5)$ & $-0.059(6)$ & $-0.013(5)$ \\
\hline C(13) & $0.23(1)$ & $0.036(4)$ & $0.122(8)$ & $-0.023(6)$ & $-0.026(8)$ & $0.011(5)$ \\
\hline $\mathrm{C}(14)$ & $0.109(7)$ & $0.094(7)$ & $0.111(7)$ & $-0.027(5)$ & $-0.028(6)$ & $-0.046(6)$ \\
\hline$C(15)$ & $0.046(3)$ & $0.038(3)$ & $0.039(3)$ & $-0.013(2)$ & $-0.009(2)$ & $0.005(2)$ \\
\hline$C(16)$ & $0.044(3)$ & $0.032(3)$ & $0.040(3)$ & $-0.012(2)$ & $-0.011(2)$ & $0.001(2)$ \\
\hline $\mathrm{C}(17)$ & $0.053(3)$ & $0.027(2)$ & $0.042(3)$ & $-0.007(2)$ & $-0.004(2)$ & $-0.005(2)$ \\
\hline C(18) & $0.043(3)$ & $0.035(3)$ & $0.054(3)$ & $-0.000(2)$ & $-0.007(2)$ & $-0.001(2)$ \\
\hline C(19) & $0.044(3)$ & $0.040(3)$ & $0.052(3)$ & $-0.006(2)$ & $-0.014(2)$ & $0.011(2)$ \\
\hline C(20) & $0.065(4)$ & $0.071(4)$ & $0.043(3)$ & $-0.029(3)$ & $-0.005(3)$ & $0.009(3)$ \\
\hline $\mathrm{C}(21)$ & $0.048(3)$ & $0.044(3)$ & $0.058(3)$ & $-0.017(3)$ & $-0.015(3)$ & $0.003(3)$ \\
\hline $\mathrm{C}(22)$ & $0.078(4)$ & $0.059(4)$ & $0.045(3)$ & $-0.017(3)$ & $0.001(3)$ & $-0.010(3)$ \\
\hline C(23) & $0.051(4)$ & $0.064(5)$ & $0.101(6)$ & $0.009(3)$ & $0.004(4)$ & $-0.012(4)$ \\
\hline $\mathrm{C}(24)$ & $0.071(4)$ & $0.070(5)$ & $0.072(4)$ & $-0.013(4)$ & $-0.034(4)$ & $0.027(4)$ \\
\hline
\end{tabular}


Table S9. Intramolecular Distances Involving the Nonhydrogen Atoms for $\left[\mathrm{Cp} * \operatorname{Ir}(\mathrm{CO})\left(\mu_{2}-\mathrm{NCN}-N, N\right)\left(\mu_{2}-\mathrm{OCNCN}\right) \operatorname{IrCp} *\right](6)$.

\begin{tabular}{|c|c|c|c|c|c|}
\hline atom & atom & distance & atom & atom & distance \\
\hline $\operatorname{Ir}(1)$ & $\mathrm{N}(3)$ & $2.078(4)$ & $\operatorname{Ir}(1)$ & $\mathrm{C}(1)$ & $2.057(6)$ \\
\hline $\operatorname{Ir}(1)$ & $\mathrm{C}(4)$ & $1.83(1)$ & $\operatorname{Ir}(1)$ & $\mathrm{C}(5)$ & $2.247(6)$ \\
\hline $\operatorname{Ir}(1)$ & $\mathrm{C}(6)$ & $2.284(6)$ & $\operatorname{Ir}(1)$ & $\mathrm{C}(7)$ & $2.287(7)$ \\
\hline $\operatorname{Ir}(1)$ & $\mathrm{C}(8)$ & $2.227(6)$ & $\operatorname{Ir}(1)$ & $\mathrm{C}(9)$ & $2.209(7)$ \\
\hline $\operatorname{Ir}(2)$ & $\mathrm{N}(1)$ & $2.052(5)$ & $\operatorname{Ir}(2)$ & $\mathrm{N}(3)$ & $1.960(4)$ \\
\hline $\operatorname{Ir}(2)$ & $C(15)$ & $2.144(6)$ & $\operatorname{Ir}(2)$ & $C(16)$ & $2.165(6)$ \\
\hline $\operatorname{Ir}(2)$ & $\mathrm{C}(17)$ & $2.149(5)$ & $\operatorname{Ir}(2)$ & $C(18)$ & $2.165(5)$ \\
\hline $\operatorname{Ir}(2)$ & C(19) & $2.207(6)$ & $\mathrm{O}(1)$ & $\mathrm{C}(1)$ & 1.209(9) \\
\hline $\mathrm{O}(2)$ & $C(4)$ & $1.16(1)$ & $\mathrm{N}(1)$ & $\mathrm{C}(1)$ & $1.407(8)$ \\
\hline $\mathrm{N}(1)$ & $\mathrm{C}(2)$ & $1.343(7)$ & $\mathrm{N}(2)$ & $\mathrm{C}(2)$ & $1.149(8)$ \\
\hline $\mathrm{N}(3)$ & $\mathrm{C}(3)$ & $1.314(7)$ & $\mathrm{N}(4)$ & $\mathrm{C}(3)$ & $1.167(8)$ \\
\hline C(5) & C(6) & $1.416(9)$ & $\mathrm{C}(5)$ & $\mathrm{C}(9)$ & $1.45(1)$ \\
\hline $\mathrm{C}(5)$ & $C(10)$ & $1.49(1)$ & $\mathrm{C}(6)$ & $\mathrm{C}(7)$ & $1.412(9)$ \\
\hline C(6) & $\mathrm{C}(11)$ & $1.49(1)$ & $\mathrm{C}(7)$ & $\mathrm{C}(8)$ & $1.43(1)$ \\
\hline C(7) & $\mathrm{C}(12)$ & 1.51(1) & $\mathrm{C}(8)$ & C(9) & 1.44(1) \\
\hline C(8) & $\mathrm{C}(13)$ & $1.51(1)$ & $\mathrm{C}(9)$ & $\mathrm{C}(14)$ & $1.49(1)$ \\
\hline C(15) & $C(16)$ & $1.456(7)$ & $C(15)$ & $C(19)$ & $1.428(7)$ \\
\hline $\mathrm{C}(15)$ & $\mathrm{C}(20)$ & $1.495(8)$ & $\mathrm{C}(16)$ & $\mathrm{C}(17)$ & $1.417(7)$ \\
\hline$C(16)$ & C(21) & $1.488(7)$ & C(17) & C(18) & $1.467(8)$ \\
\hline $\mathrm{C}(17)$ & $C(22)$ & $1.501(8)$ & $C(18)$ & $C(19)$ & $1.429(8)$ \\
\hline C(18) & $\mathrm{C}(23)$ & $1.497(8)$ & $\mathrm{C}(19)$ & $C(24)$ & $1.493(9)$ \\
\hline
\end{tabular}

Distances are in angstroms.

Table S10. Intramolecular Bond Angles Involving the Nonhydrogen Atoms for $\left[\mathrm{Cp} * \operatorname{Ir}(\mathrm{CO})\left(\mu_{2}-\mathrm{NCN}-N, N\right)\left(\mu_{2}-\mathrm{OCNCN}\right) \operatorname{IrCp} *\right](\mathbf{6})$.

\begin{tabular}{|c|c|c|c|c|c|c|c|}
\hline atom & atom & atom & angle & atom & atom & atom & angle \\
\hline $\operatorname{Ir}(1)$ & $\mathrm{N}(3)$ & $\operatorname{Ir}(2)$ & $124.2(2)$ & $\mathrm{C}(1)$ & $\operatorname{Ir}(1)$ & $\mathrm{N}(3)$ & $84.5(2)$ \\
\hline $\operatorname{Ir}(1)$ & $\mathrm{N}(3)$ & $\mathrm{C}(3)$ & 111.9(3) & $C(4)$ & $\operatorname{Ir}(1)$ & $\mathrm{N}(3)$ & $90.5(3)$ \\
\hline C(5) & $\operatorname{Ir}(1)$ & $\mathrm{N}(3)$ & $112.9(2)$ & $C(6)$ & $\operatorname{Ir}(1)$ & $\mathrm{N}(3)$ & $92.6(2)$ \\
\hline C(7) & $\operatorname{Ir}(1)$ & $\mathrm{N}(3)$ & $105.9(2)$ & $C(8)$ & $\operatorname{Ir}(1)$ & $\mathrm{N}(3)$ & 142.1(3) \\
\hline C(9) & $\operatorname{Ir}(1)$ & $\mathrm{N}(3)$ & $150.8(2)$ & $\operatorname{Ir}(1)$ & $\mathrm{C}(1)$ & $\mathrm{O}(1)$ & $123.7(5)$ \\
\hline $\operatorname{Ir}(1)$ & $\mathrm{C}(1)$ & $\mathrm{N}(1)$ & $116.3(4)$ & $C(4)$ & $\operatorname{Ir}(1)$ & $\mathrm{C}(1)$ & 88.6(3) \\
\hline C(5) & $\operatorname{Ir}(1)$ & $\mathrm{C}(1)$ & $92.4(2)$ & $\mathrm{C}(6)$ & $\operatorname{Ir}(1)$ & $\mathrm{C}(1)$ & $121.6(2)$ \\
\hline$C(7)$ & $\operatorname{Ir}(1)$ & $\mathrm{C}(1)$ & $153.8(2)$ & $\mathrm{C}(8)$ & $\operatorname{Ir}(1)$ & $C(1)$ & 131.5(3) \\
\hline C(9) & $\operatorname{Ir}(1)$ & $\mathrm{C}(1)$ & $96.5(2)$ & $\operatorname{Ir}(1)$ & $C(4)$ & $O(2)$ & $178(1)$ \\
\hline C(5) & $\operatorname{Ir}(1)$ & $C(4)$ & $156.5(3)$ & $\mathrm{C}(6)$ & $\operatorname{Ir}(1)$ & $C(4)$ & $149.8(3)$ \\
\hline C(7) & $\operatorname{Ir}(1)$ & $C(4)$ & $114.7(3)$ & $\mathrm{C}(8)$ & $\operatorname{Ir}(1)$ & $\mathrm{C}(4)$ & 100.2(3) \\
\hline C(9) & $\operatorname{Ir}(1)$ & $C(4)$ & $118.6(3)$ & $\operatorname{Ir}(1)$ & $\mathrm{C}(5)$ & C(6) & 73.2(3) \\
\hline C(6) & $\operatorname{Ir}(1)$ & $\mathrm{C}(5)$ & $36.4(2)$ & $C(7)$ & $\operatorname{Ir}(1)$ & C(5) & $61.4(2)$ \\
\hline C(8) & $\operatorname{Ir}(1)$ & $\mathrm{C}(5)$ & 62.3(2) & $\operatorname{Ir}(1)$ & $\mathrm{C}(5)$ & C(9) & 69.6(3) \\
\hline $\mathrm{C}(9)$ & $\operatorname{Ir}(1)$ & $\mathrm{C}(5)$ & $38.0(3)$ & $\operatorname{Ir}(1)$ & $\mathrm{C}(5)$ & $C(10)$ & $125.5(4)$ \\
\hline $\operatorname{Ir}(1)$ & C(6) & $\mathrm{C}(5)$ & $70.4(3)$ & $\operatorname{Ir}(1)$ & $\mathrm{C}(6)$ & $\mathrm{C}(7)$ & $72.1(4)$ \\
\hline$C(7)$ & $\operatorname{Ir}(1)$ & $\mathrm{C}(6)$ & $36.0(2)$ & $\mathrm{C}(8)$ & $\operatorname{Ir}(1)$ & C(6) & $60.8(3)$ \\
\hline C(9) & $\operatorname{Ir}(1)$ & $\mathrm{C}(6)$ & $62.0(3)$ & $\operatorname{Ir}(1)$ & $\mathrm{C}(6)$ & $C(11)$ & $123.7(4)$ \\
\hline $\operatorname{Ir}(1)$ & $\mathrm{C}(7)$ & $\mathrm{C}(6)$ & 71.9(3) & $\operatorname{Ir}(1)$ & $\mathrm{C}(7)$ & $\mathrm{C}(8)$ & 69.3(4) \\
\hline $\mathrm{C}(8)$ & $\operatorname{Ir}(1)$ & $\mathrm{C}(7)$ & $36.8(3)$ & $\mathrm{C}(9)$ & $\operatorname{Ir}(1)$ & $\mathrm{C}(7)$ & $62.6(3)$ \\
\hline $\operatorname{Ir}(1)$ & $\mathrm{C}(7)$ & $\mathrm{C}(12)$ & $129.1(5)$ & $\operatorname{Ir}(1)$ & $\mathrm{C}(8)$ & $\mathrm{C}(7)$ & $73.9(3)$ \\
\hline $\operatorname{Ir}(1)$ & $\mathrm{C}(8)$ & $\mathrm{C}(9)$ & $70.4(4)$ & C(9) & $\operatorname{Ir}(1)$ & $\mathrm{C}(8)$ & $37.9(3)$ \\
\hline $\operatorname{Ir}(1)$ & $\mathrm{C}(8)$ & $C(13)$ & $124.9(6)$ & $\operatorname{Ir}(1)$ & $\mathrm{C}(9)$ & C(5) & $72.4(4)$ \\
\hline $\operatorname{Ir}(1)$ & $\mathrm{C}(9)$ & $\mathrm{C}(8)$ & $71.7(4)$ & $\operatorname{Ir}(1)$ & $\mathrm{C}(9)$ & $C(14)$ & $126.2(5)$ \\
\hline $\mathrm{N}(3)$ & $\operatorname{Ir}(2)$ & $\mathrm{N}(1)$ & $82.1(2)$ & $\operatorname{Ir}(2)$ & $\mathrm{N}(1)$ & $\mathrm{C}(1)$ & $127.4(4)$ \\
\hline $\operatorname{Ir}(2)$ & $\mathrm{N}(1)$ & $C(2)$ & $118.2(4)$ & C(15) & $\operatorname{Ir}(2)$ & $\mathrm{N}(1)$ & $126.2(2)$ \\
\hline $\mathrm{C}(16)$ & $\operatorname{Ir}(2)$ & $\mathrm{N}(1)$ & $165.5(2)$ & $C(17)$ & $\operatorname{Ir}(2)$ & $\mathrm{N}(1)$ & $148.9(2)$ \\
\hline
\end{tabular}




\begin{tabular}{|c|c|c|c|c|c|c|c|}
\hline$C(18)$ & $\operatorname{Ir}(2)$ & $\mathrm{N}(1)$ & $113.7(2)$ & $C(19)$ & $\operatorname{Ir}(2)$ & $\mathrm{N}(1)$ & $105.1(2)$ \\
\hline $\operatorname{Ir}(2)$ & $\mathrm{N}(3)$ & $\mathrm{C}(3)$ & $122.5(4)$ & C(15) & $\operatorname{Ir}(2)$ & $\mathrm{N}(3)$ & $131.4(2)$ \\
\hline C(16) & $\operatorname{Ir}(2)$ & $\mathrm{N}(3)$ & 106.6(2) & $\mathrm{C}(17)$ & $\operatorname{Ir}(2)$ & $\mathrm{N}(3)$ & $112.8(2)$ \\
\hline$C(18)$ & $\operatorname{Ir}(2)$ & N(3) & $145.6(2)$ & $C(19)$ & $\operatorname{Ir}(2)$ & $\mathrm{N}(3)$ & 169.7(2) \\
\hline $\operatorname{Ir}(2)$ & $C(15)$ & $C(16)$ & 71.0(3) & $C(16)$ & $\operatorname{Ir}(2)$ & $C(15)$ & 39.5(2) \\
\hline $\mathrm{C}(17)$ & $\operatorname{Ir}(2)$ & $C(15)$ & $65.0(2)$ & $\mathrm{C}(18)$ & $\operatorname{Ir}(2)$ & $\mathrm{C}(15)$ & $64.9(2)$ \\
\hline $\operatorname{Ir}(2)$ & $C(15)$ & C(19) & 73.3(3) & $\mathrm{C}(19)$ & $\operatorname{Ir}(2)$ & $C(15)$ & $38.3(2)$ \\
\hline $\operatorname{Ir}(2)$ & $C(15)$ & $C(20)$ & $125.2(4)$ & $\operatorname{Ir}(2)$ & $\mathrm{C}(16)$ & $C(15)$ & $69.5(3)$ \\
\hline $\operatorname{Ir}(2)$ & $\mathrm{C}(16)$ & $\mathrm{C}(17)$ & 70.2(3) & $C(17)$ & $\operatorname{Ir}(2)$ & $\mathrm{C}(16)$ & $38.4(2)$ \\
\hline C(18) & $\operatorname{Ir}(2)$ & $C(16)$ & $65.5(2)$ & $C(19)$ & $\operatorname{Ir}(2)$ & $C(16)$ & $64.9(2)$ \\
\hline $\operatorname{Ir}(2)$ & $C(16)$ & $C(21)$ & $126.7(4)$ & $\operatorname{Ir}(2)$ & $C(17)$ & $C(16)$ & 71.4(3) \\
\hline $\operatorname{Ir}(2)$ & $C(17)$ & $C(18)$ & 70.7(3) & $C(18)$ & $\operatorname{Ir}(2)$ & $\mathrm{C}(17)$ & 39.7(2) \\
\hline C(19) & $\operatorname{Ir}(2)$ & $C(17)$ & $64.7(2)$ & $\operatorname{Ir}(2)$ & $\mathrm{C}(17)$ & $\mathrm{C}(22)$ & 126.3(4) \\
\hline $\operatorname{Ir}(2)$ & $\mathrm{C}(18)$ & $C(17)$ & $69.5(3)$ & $\operatorname{Ir}(2)$ & $\mathrm{C}(18)$ & $\mathrm{C}(19)$ & 72.5(3) \\
\hline C(19) & $\operatorname{Ir}(2)$ & $C(18)$ & $38.1(2)$ & $\operatorname{Ir}(2)$ & C(18) & $C(23)$ & $126.2(5)$ \\
\hline $\operatorname{Ir}(2)$ & C(19) & $C(15)$ & 68.4(3) & $\operatorname{Ir}(2)$ & C(19) & C(18) & 69.4(3) \\
\hline $\operatorname{Ir}(2)$ & $\mathrm{C}(19)$ & $C(24)$ & 128.6(5) & $\mathrm{O}(1)$ & $\mathrm{C}(1)$ & $\mathrm{N}(1)$ & $120.0(5)$ \\
\hline $\mathrm{C}(2)$ & $\mathrm{N}(1)$ & $\mathrm{C}(1)$ & $114.4(5)$ & $\mathrm{N}(1)$ & $C(2)$ & $\mathrm{N}(2)$ & $177.4(7)$ \\
\hline $\mathrm{N}(3)$ & $\mathrm{C}(3)$ & $\mathrm{N}(4)$ & $179.3(7)$ & C(5) & $C(6)$ & $C(7)$ & 109.9(6) \\
\hline C(9) & C(5) & $\mathrm{C}(6)$ & $107.6(5)$ & $\mathrm{C}(10)$ & $\mathrm{C}(5)$ & C(6) & 126.7(7) \\
\hline C(5) & $\mathrm{C}(6)$ & $\mathrm{C}(11)$ & $125.0(6)$ & C(5) & $\mathrm{C}(9)$ & $\mathrm{C}(8)$ & 106.3(6) \\
\hline$C(10)$ & $\mathrm{C}(5)$ & $\mathrm{C}(9)$ & $125.6(7)$ & C(5) & $\mathrm{C}(9)$ & $C(14)$ & 125.8(6) \\
\hline$C(6)$ & $C(7)$ & $\mathrm{C}(8)$ & $107.0(6)$ & $C(11)$ & $C(6)$ & $\mathrm{C}(7)$ & 125.1(6) \\
\hline $\mathrm{C}(6)$ & $C(7)$ & $\mathrm{C}(12)$ & $127.0(7)$ & $C(7)$ & $\mathrm{C}(8)$ & $\mathrm{C}(9)$ & 109.2(6) \\
\hline $\mathrm{C}(12)$ & $C(7)$ & $\mathrm{C}(8)$ & $125.6(6)$ & $C(7)$ & $\mathrm{C}(8)$ & $C(13)$ & 126.9(8) \\
\hline C(13) & $\mathrm{C}(8)$ & C(9) & 123.8(8) & C(8) & C(9) & $\mathrm{C}(14)$ & $127.5(7)$ \\
\hline$C(15)$ & $\mathrm{C}(16)$ & $C(17)$ & $106.9(4)$ & $\mathrm{C}(19)$ & $\mathrm{C}(15)$ & $C(16)$ & $108.9(4)$ \\
\hline$C(20)$ & $\mathrm{C}(15)$ & $C(16)$ & $125.0(5)$ & $C(15)$ & $\mathrm{C}(16)$ & $\mathrm{C}(21)$ & $126.0(4)$ \\
\hline C(15) & C(19) & $C(18)$ & $108.0(5)$ & $C(20)$ & $C(15)$ & C(19) & $126.0(5)$ \\
\hline C(15) & $C(19)$ & $C(24)$ & $125.5(5)$ & $C(16)$ & $C(17)$ & C(18) & 108.7(4) \\
\hline $\mathrm{C}(21)$ & C(16) & $C(17)$ & 127.1(5) & $C(16)$ & C(17) & $\mathrm{C}(22)$ & $125.6(5)$ \\
\hline $\mathrm{C}(17)$ & $\mathrm{C}(18)$ & C(19) & $107.3(4)$ & $\mathrm{C}(22)$ & $\mathrm{C}(17)$ & C(18) & 125.6(5) \\
\hline $\mathrm{C}(17)$ & $\mathrm{C}(18)$ & $\mathrm{C}(23)$ & $125.8(5)$ & $\mathrm{C}(23)$ & $\mathrm{C}(18)$ & $\mathrm{C}(19)$ & 126.8(5) \\
\hline C(18) & $\mathrm{C}(19)$ & $C(24)$ & $126.4(5)$ & & & & \\
\hline
\end{tabular}

Angles are in degrees. 
Table S11. Positional Parameters and B(eq) for [Cp* $\left.\operatorname{Ir}\left(\mathrm{PMe}_{3}\right)\left(\mu_{2}-\mathrm{NCN}-N, N\right)_{2} \operatorname{IrCp} *\right]$ (8d).

\begin{tabular}{|c|c|c|c|c|c|}
\hline atom & $\mathrm{X}$ & $\mathrm{y}$ & $\mathrm{Z}$ & $B(e q)$ & occupancy \\
\hline $\operatorname{Ir}(1)$ & $0.22217(2)$ & 0.2500 & $0.03820(4)$ & $0.63(1)$ & $1 / 2$ \\
\hline $\operatorname{Ir}(2)$ & $0.06294(2)$ & 0.2500 & $0.16486(4)$ & $0.68(1)$ & $1 / 2$ \\
\hline $\mathrm{P}(1)$ & $0.1780(2)$ & 0.2500 & $-0.1769(3)$ & $0.94(6)$ & $1 / 2$ \\
\hline $\mathrm{N}(1)$ & $0.1370(4)$ & $0.1611(5)$ & $0.0944(6)$ & $1.0(1)$ & \\
\hline $\mathrm{N}(2)$ & $0.1437(4)$ & $-0.0171(5)$ & $0.1324(7)$ & $1.5(1)$ & \\
\hline $\mathrm{C}(1)$ & $0.1404(4)$ & $0.0682(6)$ & $0.1149(7)$ & $0.7(1)$ & \\
\hline $\mathrm{C}(2)$ & $0.3336(7)$ & 0.2500 & $-0.015(1)$ & $1.3(2)$ & $1 / 2$ \\
\hline C(3) & $0.3171(4)$ & $0.1658(6)$ & $0.0665(8)$ & $1.2(2)$ & \\
\hline C(4) & $0.2956(4)$ & $0.1986(6)$ & $0.2009(8)$ & $1.0(1)$ & \\
\hline C(5) & $0.3673(8)$ & $0.25000(1)$ & $-0.152(1)$ & 2.1(3) & $1 / 2$ \\
\hline C(6) & $0.3268(5)$ & $0.0617(7)$ & $0.0210(8)$ & $1.6(2)$ & \\
\hline C(7) & $0.2720(5)$ & $0.1323(6)$ & $0.3161(9)$ & $1.3(2)$ & \\
\hline C(8) & $0.0018(4)$ & $0.1963(6)$ & $0.3332(7)$ & $0.8(2)$ & \\
\hline C(9) & $-0.0306(5)$ & $0.1631(6)$ & $0.2084(8)$ & $1.1(2)$ & \\
\hline C(10) & $-0.0474(6)$ & 0.2500 & $0.128(1)$ & $1.2(2)$ & $1 / 2$ \\
\hline $\mathrm{C}(11)$ & $0.0313(5)$ & $0.1312(6)$ & $0.4429(7)$ & $1.4(2)$ & \\
\hline $\mathrm{C}(12)$ & $-0.0406(4)$ & $0.0609(6)$ & $0.1676(8)$ & $1.3(2)$ & \\
\hline C(13) & $-0.0815(8)$ & $0.25000(1)$ & $-0.007(1)$ & $1.9(3)$ & $1 / 2$ \\
\hline $\mathrm{C}(14)$ & $0.0839(6)$ & $0.25000(1)$ & $-0.191(1)$ & $1.8(3)$ & $1 / 2$ \\
\hline C(15) & $0.2034(5)$ & $0.1464(6)$ & $-0.279(1)$ & $2.0(2)$ & \\
\hline $\mathrm{H}(1)$ & 0.3726 & 0.1844 & -0.1826 & 2.493 & $1 / 2$ \\
\hline $\mathrm{H}(2)$ & 0.3400 & 0.2858 & -0.2156 & 2.502 & $1 / 2$ \\
\hline $\mathrm{H}(3)$ & 0.4118 & 0.2798 & -0.1432 & 2.497 & $1 / 2$ \\
\hline $\mathrm{H}(4)$ & 0.3750 & 0.0507 & 0.0059 & 1.901 & \\
\hline $\mathrm{H}(5)$ & 0.3018 & 0.0491 & -0.0610 & 1.900 & \\
\hline $\mathrm{H}(6)$ & 0.3107 & 0.0192 & 0.0912 & 1.892 & \\
\hline $\mathrm{H}(7)$ & 0.3092 & 0.0895 & 0.3396 & 1.603 & \\
\hline $\mathrm{H}(8)$ & 0.2327 & 0.0947 & 0.2893 & 1.606 & \\
\hline $\mathrm{H}(9)$ & 0.2603 & 0.1716 & 0.3929 & 1.606 & \\
\hline $\mathrm{H}(10)$ & -0.0056 & 0.0926 & 0.4785 & 1.664 & \\
\hline $\mathrm{H}(11)$ & 0.0505 & 0.1704 & 0.5140 & 1.667 & \\
\hline $\mathrm{H}(12)$ & 0.0664 & 0.0894 & 0.4069 & 1.663 & \\
\hline $\mathrm{H}(13)$ & -0.0688 & 0.0291 & 0.2340 & 1.537 & \\
\hline $\mathrm{H}(14)$ & -0.0630 & 0.0583 & 0.0810 & 1.529 & \\
\hline $\mathrm{H}(15)$ & 0.0032 & 0.0288 & 0.1621 & 1.534 & \\
\hline $\mathrm{H}(16)$ & -0.0886 & 0.3157 & -0.0360 & 2.330 & $1 / 2$ \\
\hline $\mathrm{H}(17)$ & -0.0522 & 0.2172 & -0.0704 & 2.331 & $1 / 2$ \\
\hline H(18) & -0.1250 & 0.2172 & -0.0017 & 2.328 & $1 / 2$ \\
\hline H(19) & 0.0660 & 0.3100 & -0.1560 & 2.150 & $1 / 2$ \\
\hline $\mathrm{H}(20)$ & 0.0712 & 0.2500 & -0.2842 & 2.150 & $1 / 2$ \\
\hline $\mathrm{H}(21)$ & 0.0653 & 0.1969 & -0.1395 & 2.150 & $1 / 2$ \\
\hline $\mathrm{H}(22)$ & 0.2526 & 0.1386 & -0.2786 & 2.355 & \\
\hline $\mathrm{H}(23)$ & 0.1879 & 0.1559 & -0.3705 & 2.356 & \\
\hline $\mathrm{H}(24)$ & 0.1822 & 0.0893 & -0.2422 & 2.366 & \\
\hline
\end{tabular}


Table S12. Anisotropic Displacement Parameters for [Cp* $\left.\operatorname{Ir}\left(\mathrm{PMe}_{3}\right)\left(\mu_{2}-\mathrm{NCN}-N, N\right)_{2} \operatorname{IrCp}{ }^{*}\right]$ (8d).

\begin{tabular}{|c|c|c|c|c|c|c|}
\hline atom & U11 & U22 & U33 & U12 & U13 & U23 \\
\hline $\operatorname{Ir}(1)$ & $0.0084(3)$ & $0.0061(3)$ & $0.0095(3)$ & 0.0000 & $0.0001(1)$ & 0.0000 \\
\hline $\operatorname{Ir}(2)$ & $0.0085(3)$ & $0.0075(3)$ & $0.0100(3)$ & 0.0000 & $-0.0000(1)$ & 0.0000 \\
\hline $\mathrm{P}(1)$ & $0.015(1)$ & $0.010(1)$ & $0.010(1)$ & 0.0000 & $-0.001(1)$ & 0.0000 \\
\hline $\mathrm{N}(1)$ & $0.017(4)$ & $0.010(4)$ & $0.011(3)$ & $-0.004(3)$ & $0.003(3)$ & $0.005(3)$ \\
\hline $\mathrm{N}(2)$ & $0.020(4)$ & $0.010(4)$ & $0.028(4)$ & 0.001(3) & $-0.006(3)$ & $0.001(3)$ \\
\hline $\mathrm{C}(1)$ & $0.007(4)$ & $0.014(4)$ & $0.007(3)$ & $0.003(3)$ & $-0.000(3)$ & $0.002(3)$ \\
\hline $\mathrm{C}(2)$ & $0.023(7)$ & $0.010(6)$ & $0.018(6)$ & 0.0000 & $0.018(5)$ & 0.0000 \\
\hline C(3) & $0.014(4)$ & $0.013(4)$ & $0.017(4)$ & $0.002(3)$ & $-0.000(3)$ & $0.010(3)$ \\
\hline C(4) & $0.007(4)$ & $0.017(4)$ & $0.013(4)$ & $0.000(3)$ & $0.003(3)$ & $0.002(3)$ \\
\hline C(5) & $0.033(8)$ & $0.031(8)$ & $0.015(6)$ & 0.0000 & $0.015(6)$ & 0.0000 \\
\hline C(6) & $0.020(5)$ & $0.027(5)$ & $0.013(4)$ & $0.005(4)$ & $0.005(3)$ & $-0.004(4)$ \\
\hline C(7) & $0.019(4)$ & $0.011(4)$ & $0.021(4)$ & $-0.008(3)$ & $0.005(3)$ & $0.000(4)$ \\
\hline $\mathrm{C}(8)$ & $0.009(4)$ & $0.010(4)$ & $0.011(4)$ & $0.004(3)$ & $0.001(3)$ & $-0.001(3)$ \\
\hline C(9) & $0.016(4)$ & $0.013(4)$ & $0.013(4)$ & $-0.008(3)$ & $-0.006(3)$ & $0.003(4)$ \\
\hline $\mathrm{C}(10)$ & $0.014(5)$ & $0.008(6)$ & $0.024(6)$ & 0.0000 & $0.016(5)$ & 0.0000 \\
\hline $\mathrm{C}(11)$ & $0.030(5)$ & $0.013(4)$ & $0.009(3)$ & $0.001(4)$ & $-0.001(4)$ & $0.003(3)$ \\
\hline $\mathrm{C}(12)$ & $0.009(4)$ & $0.013(4)$ & $0.026(5)$ & $-0.000(3)$ & $0.008(3)$ & $-0.002(3)$ \\
\hline C(13) & $0.036(8)$ & $0.026(8)$ & $0.012(6)$ & 0.0000 & $0.003(6)$ & 0.0000 \\
\hline$C(14)$ & $0.006(6)$ & $0.047(9)$ & $0.014(6)$ & 0.0000 & $-0.005(5)$ & 0.0000 \\
\hline $\mathrm{C}(15)$ & $0.033(5)$ & $0.014(4)$ & $0.028(5)$ & $0.004(4)$ & $-0.003(4)$ & $-0.013(4)$ \\
\hline
\end{tabular}

Table S13. Intramolecular Distances Involving the Nonhydrogen Atoms for $\left[\mathrm{Cp}^{*} \operatorname{Ir}\left(\mathrm{PMe}_{3}\right)\left(\mu_{2}-\mathrm{NCN}-N, N\right)_{2} \operatorname{IrCp} *\right]$ (8d).

\begin{tabular}{lll|lll}
\hline atom & atom & distance & atom & atom & distance \\
\hline $\operatorname{Ir}(1)$ & $\mathrm{P}(1)$ & $2.268(3)$ & $\operatorname{Ir}(1)$ & $\mathrm{N}(1)$ & $2.113(7)$ \\
$\operatorname{Ir}(1)$ & $\mathrm{N}(1)^{1)}$ & $2.113(7)$ & $\operatorname{Ir}(1)$ & $\mathrm{C}(2)$ & $2.20(1)$ \\
$\operatorname{Ir}(1)$ & $\mathrm{C}(3)$ & $2.176(8)$ & $\operatorname{Ir}(1)$ & $\mathrm{C}(3)^{1)}$ & $2.176(8)$ \\
$\operatorname{Ir}(1)$ & $\mathrm{C}(4)$ & $2.240(8)$ & $\operatorname{Ir}(1)$ & $\mathrm{C}(4)^{1)}$ & $2.240(8)$ \\
$\operatorname{Ir}(2)$ & $\mathrm{N}(1)$ & $1.996(7)$ & $\operatorname{Ir}(2)$ & $\mathrm{N}(1)^{1)}$ & $1.996(7)$ \\
$\operatorname{Ir}(2)$ & $\mathrm{C}(8)$ & $2.153(7)$ & $\operatorname{Ir}(2)$ & $\mathrm{C}(8)^{1)}$ & $2.153(7)$ \\
$\operatorname{Ir}(2)$ & $\mathrm{C}(9)$ & $2.197(8)$ & $\operatorname{Ir}(2)$ & $\mathrm{C}(9)^{1)}$ & $2.197(8)$ \\
$\operatorname{Ir}(2)$ & $\mathrm{C}(10)$ & $2.15(1)$ & $\mathrm{P}(1)$ & $\mathrm{C}(14)$ & $1.81(1)$ \\
$\mathrm{P}(1)$ & $\mathrm{C}(15)$ & $1.806(9)$ & $\mathrm{P}(1)$ & $\mathrm{C}(15)^{1)}$ & $1.806(9)$ \\
$\mathrm{N}(1)$ & $\mathrm{C}(1)$ & $1.29(1)$ & $\mathrm{N}(2)$ & $\mathrm{C}(1)$ & $1.18(1)$ \\
$\mathrm{C}(2)$ & $\mathrm{C}(3)$ & $1.44(1)$ & $\mathrm{C}(2)$ & $\mathrm{C}(3)^{1)}$ & $1.44(1)$ \\
$\mathrm{C}(2)$ & $\mathrm{C}(5)$ & $1.49(2)$ & $\mathrm{C}(3)$ & $\mathrm{C}(4)$ & $1.45(1)$ \\
$\mathrm{C}(3)$ & $\mathrm{C}(6)$ & $1.51(1)$ & $\mathrm{C}(4)$ & $\mathrm{C}(4)^{1)}$ & $1.41(1)$ \\
$\mathrm{C}(4)$ & $\mathrm{C}(7)$ & $1.52(1)$ & $\mathrm{C}(8)$ & $\mathrm{C}(8)^{1)}$ & $1.47(1)$ \\
$\mathrm{C}(8)$ & $\mathrm{C}(9)$ & $1.44(1)$ & $\mathrm{C}(8)$ & $\mathrm{C}(11)$ & $1.51(1)$ \\
$\mathrm{C}(9)$ & $\mathrm{C}(10)$ & $1.47(1)$ & $\mathrm{C}(9)$ & $\mathrm{C}(12)$ & $1.47(1)$ \\
$\mathrm{C}(10)$ & $\mathrm{C}(9)^{1)}$ & $1.47(1)$ & $\mathrm{C}(10)$ & $\mathrm{C}(13)$ & $1.47(2)$ \\
$\operatorname{Ir}(1)$ & $\mathrm{P}(1)$ & $2.268(3)$ & $\operatorname{Ir}(1)$ & $\mathrm{N}(1)$ & $2.113(7)$ \\
$\operatorname{Ir}(1)$ & $\mathrm{N}(1)^{1)}$ & $2.113(7)$ & $\operatorname{Ir}(1)$ & $\mathrm{C}(2)$ & $2.20(1)$ \\
\hline $\mathrm{Din}$ & & &
\end{tabular}

Distances are in angstroms. Symmetry operations, 1$):-\mathrm{X},-\mathrm{Y}+1 / 2, \mathrm{Z}$.

Table S14. Intramolecular Bond Angles Involving the Nonhydrogen Atoms for $\left[\mathrm{Cp} * \operatorname{Ir}\left(\mathrm{PMe}_{3}\right)\left(\mu_{2}-\mathrm{NCN}-N, N\right)_{2} \operatorname{IrCp} *\right](\mathbf{8 d})$.

\begin{tabular}{llll|lllr}
\hline atom & atom & atom & angle & atom & atom & atom & \multicolumn{1}{c}{ angle } \\
\hline $\mathrm{N}(1)$ & $\operatorname{Ir}(1)$ & $\mathrm{P}(1)$ & $87.2(2)$ & $\mathrm{N}(1)^{1)}$ & $\operatorname{Ir}(1)$ & $\mathrm{P}(1)$ & $87.2(2)$ \\
$\mathrm{C}(2)$ & $\operatorname{Ir}(1)$ & $\mathrm{P}(1)$ & $98.3(3)$ & $\mathrm{C}(3)$ & $\operatorname{Ir}(1)$ & $\mathrm{P}(1)$ & $115.6(2)$ \\
$\mathrm{C}(3)^{1)}$ & $\operatorname{Ir}(1)$ & $\mathrm{P}(1)$ & $115.6(2)$ & $\mathrm{C}(4)$ & $\operatorname{Ir}(1)$ & $\mathrm{P}(1)$ & $153.4(2)$ \\
$\mathrm{C}(4)^{1)}$ & $\operatorname{Ir}(1)$ & $\mathrm{P}(1)$ & $153.4(2)$ & $\operatorname{Ir}(1)$ & $\mathrm{P}(1)$ & $\mathrm{C}(14)$ & $116.3(4)$ \\
$\operatorname{Ir}(1)$ & $\mathrm{P}(1)$ & $\mathrm{C}(15)$ & $114.4(3)$ & $\operatorname{Ir}(1)$ & $\mathrm{P}(1)$ & $\mathrm{C}(15)^{1)}$ & $114.4(3)$
\end{tabular}




\begin{tabular}{|c|c|c|c|c|c|c|c|}
\hline $\operatorname{Ir}(1)$ & $\mathrm{N}(1)$ & $\operatorname{Ir}(2)$ & 106.8(3) & $\mathrm{N}(1)^{1)}$ & $\operatorname{Ir}(1)$ & $\mathrm{N}(1)$ & 70.5(3) \\
\hline $\operatorname{Ir}(1)$ & $\mathrm{N}(1)$ & $\mathrm{C}(1)$ & $124.8(5)$ & $C(2)$ & $\operatorname{Ir}(1)$ & $\mathrm{N}(1)$ & $144.4(2)$ \\
\hline C(3) & $\operatorname{Ir}(1)$ & $\mathrm{N}(1)$ & 108.0(3) & $C(3)^{1)}$ & $\operatorname{Ir}(1)$ & $\mathrm{N}(1)$ & 157.2(3) \\
\hline C(4) & $\operatorname{Ir}(1)$ & $\mathrm{N}(1)$ & 96.9(3) & $C(4)^{1)}$ & $\operatorname{Ir}(1)$ & $\mathrm{N}(1)$ & 118.9(3) \\
\hline $\operatorname{Ir}(1)$ & $\mathrm{N}(1)^{1)}$ & $\operatorname{Ir}(2)$ & 106.8(3) & $\operatorname{Ir}(1)$ & $\mathrm{N}(1)^{1)}$ & $C(1)^{1)}$ & $124.8(5)$ \\
\hline $\mathrm{C}(2)$ & $\operatorname{Ir}(1)$ & $\mathrm{N}(1)^{1)}$ & $144.4(2)$ & C(3) & $\operatorname{Ir}(1)$ & $\mathrm{N}(1)^{1)}$ & 157.2(3) \\
\hline$C(3)^{1)}$ & $\operatorname{Ir}(1)$ & $\mathrm{N}(1)^{1)}$ & 108.0(3) & C(4) & $\operatorname{Ir}(1)$ & $\mathrm{N}(1)^{1)}$ & 118.9(3) \\
\hline$C(4)^{1)}$ & $\operatorname{Ir}(1)$ & $\mathrm{N}(1)^{1)}$ & $96.9(3)$ & $\operatorname{Ir}(1)$ & $\mathrm{C}(2)$ & C(3) & 69.8(6) \\
\hline C(3) & $\operatorname{Ir}(1)$ & $\mathrm{C}(2)$ & $38.4(3)$ & $\operatorname{Ir}(1)$ & $\mathrm{C}(2)$ & $C(3)^{1)}$ & 69.8(6) \\
\hline$C(3)^{1)}$ & $\operatorname{Ir}(1)$ & $\mathrm{C}(2)$ & $38.4(3)$ & C(4) & $\operatorname{Ir}(1)$ & $\mathrm{C}(2)$ & $63.7(4)$ \\
\hline$C(4)^{1)}$ & $\operatorname{Ir}(1)$ & $\mathrm{C}(2)$ & $63.7(4)$ & $\operatorname{Ir}(1)$ & $\mathrm{C}(2)$ & C(5) & 129.5(9) \\
\hline $\operatorname{Ir}(1)$ & C(3) & $\mathrm{C}(2)$ & 71.8(6) & $C(3)^{1)}$ & $\operatorname{Ir}(1)$ & $\mathrm{C}(3)$ & 64.1(3) \\
\hline $\operatorname{Ir}(1)$ & C(3) & $C(4)$ & 73.2(5) & $\mathrm{C}(4)$ & $\operatorname{Ir}(1)$ & $\mathrm{C}(3)$ & 38.3(3) \\
\hline$C(4)^{1)}$ & $\operatorname{Ir}(1)$ & C(3) & 63.2(3) & $\operatorname{Ir}(1)$ & C(3) & $C(6)$ & $124.6(6)$ \\
\hline $\operatorname{Ir}(1)$ & $\mathrm{C}(3)^{1)}$ & $C(2)$ & 71.8(6) & $C(4)$ & $\operatorname{Ir}(1)$ & $C(3)^{1)}$ & 63.2(3) \\
\hline $\operatorname{Ir}(1)$ & $\mathrm{C}(3)^{1)}$ & $C(4)^{1)}$ & 73.2(5) & $C(4)^{1)}$ & $\operatorname{Ir}(1)$ & $\mathrm{C}(3)^{1)}$ & $38.3(3)$ \\
\hline $\operatorname{Ir}(1)$ & $C(3)^{1)}$ & $C(6)^{1)}$ & $124.6(6)$ & $\operatorname{Ir}(1)$ & C(4) & C(3) & $68.5(4)$ \\
\hline $\operatorname{Ir}(1)$ & $C(4)$ & $C(4)^{1)}$ & 71.6(5) & $C(4)^{1)}$ & $\operatorname{Ir}(1)$ & $C(4)$ & 36.7(3) \\
\hline $\operatorname{Ir}(1)$ & $\mathrm{C}(4)$ & $\mathrm{C}(7)$ & $122.0(5)$ & $\operatorname{Ir}(1)$ & $C(4)^{1)}$ & $C(3)^{1)}$ & $68.5(4)$ \\
\hline $\operatorname{Ir}(1)$ & $C(4)^{1)}$ & C(4) & 71.6(5) & $\operatorname{Ir}(1)$ & $C(4)^{1)}$ & $C(7)^{1)}$ & $122.0(5)$ \\
\hline $\mathrm{N}(1)^{1)}$ & $\operatorname{Ir}(2)$ & $\mathrm{N}(1)$ & 75.3(3) & $\operatorname{Ir}(2)$ & $\mathrm{N}(1)$ & C(1) & $125.8(5)$ \\
\hline C(8) & $\operatorname{Ir}(2)$ & $\mathrm{N}(1)$ & 116.4(3) & $\mathrm{C}(8)^{1)}$ & $\operatorname{Ir}(2)$ & $\mathrm{N}(1)$ & 149.5(3) \\
\hline C(9) & $\operatorname{Ir}(2)$ & $\mathrm{N}(1)$ & 108.5(3) & $C(9)^{1)}$ & $\operatorname{Ir}(2)$ & $\mathrm{N}(1)$ & $168.7(3)$ \\
\hline $\mathrm{C}(10)$ & $\operatorname{Ir}(2)$ & $\mathrm{N}(1)$ & $130.0(3)$ & $\operatorname{Ir}(2)$ & $\mathrm{N}(1)^{1)}$ & $C(1)^{1)}$ & $125.8(5)$ \\
\hline C(8) & $\operatorname{Ir}(2)$ & $\mathrm{N}(1)^{1)}$ & 149.5(3) & $C(8)^{1)}$ & $\operatorname{Ir}(2)$ & $\mathrm{N}(1)^{1)}$ & $116.4(3)$ \\
\hline C(9) & $\operatorname{Ir}(2)$ & $\mathrm{N}(1)^{1)}$ & 168.7(3) & $\mathrm{C}(9)^{1)}$ & $\operatorname{Ir}(2)$ & $\mathrm{N}(1)^{1)}$ & 108.5(3) \\
\hline$C(10)$ & $\operatorname{Ir}(2)$ & $\mathrm{N}(1)^{1)}$ & $130.0(3)$ & $\operatorname{Ir}(2)$ & $\mathrm{C}(8)$ & $C(8)^{1)}$ & $70.0(4)$ \\
\hline$C(8)^{1)}$ & $\operatorname{Ir}(2)$ & $\mathrm{C}(8)$ & $40.0(3)$ & $\operatorname{Ir}(2)$ & $\mathrm{C}(8)$ & C(9) & $72.3(4)$ \\
\hline $\mathrm{C}(9)$ & $\operatorname{Ir}(2)$ & $\mathrm{C}(8)$ & $38.8(3)$ & $\mathrm{C}(9)^{1)}$ & $\operatorname{Ir}(2)$ & $\mathrm{C}(8)$ & $65.9(3)$ \\
\hline $\mathrm{C}(10)$ & $\operatorname{Ir}(2)$ & C(8) & 65.9(4) & $\operatorname{Ir}(2)$ & $\mathrm{C}(8)$ & $\mathrm{C}(11)$ & $122.8(5)$ \\
\hline $\operatorname{Ir}(2)$ & $C(8)^{1)}$ & $\mathrm{C}(8)$ & $70.0(4)$ & C(9) & $\operatorname{Ir}(2)$ & $C(8)^{1)}$ & 65.9(3) \\
\hline $\operatorname{Ir}(2)$ & $C(8)^{1)}$ & $C(9)^{1)}$ & $72.3(4)$ & $C(9)^{1)}$ & $\operatorname{Ir}(2)$ & $C(8)^{1)}$ & $38.8(3)$ \\
\hline$C(10)$ & $\operatorname{Ir}(2)$ & $C(8)^{1)}$ & 65.9(4) & $\operatorname{Ir}(2)$ & $C(8)^{1)}$ & $\mathrm{C}(11)^{1)}$ & $122.8(5)$ \\
\hline $\operatorname{Ir}(2)$ & C(9) & C(8) & 69.0(4) & $\mathrm{C}(9)^{1)}$ & $\operatorname{Ir}(2)$ & $\mathrm{C}(9)$ & 65.7(3) \\
\hline $\operatorname{Ir}(2)$ & C(9) & C(10) & $68.5(5)$ & C(10) & $\operatorname{Ir}(2)$ & C(9) & $39.4(3)$ \\
\hline $\operatorname{Ir}(2)$ & C(9) & C(12) & $124.9(6)$ & $\operatorname{Ir}(2)$ & $C(9)^{1)}$ & $C(8)^{1)}$ & $69.0(4)$ \\
\hline $\operatorname{Ir}(2)$ & $\mathrm{C}(9)^{1)}$ & C(10) & $68.5(5)$ & C(10) & $\operatorname{Ir}(2)$ & $C(9)^{1)}$ & $39.4(3)$ \\
\hline $\operatorname{Ir}(2)$ & $\mathrm{C}(9)^{1)}$ & $C(12)^{1)}$ & $124.9(6)$ & $\operatorname{Ir}(2)$ & $\mathrm{C}(10)$ & C(9) & 72.1(6) \\
\hline $\operatorname{Ir}(2)$ & $\mathrm{C}(10)$ & $C(9)^{1)}$ & 72.1(6) & $\operatorname{Ir}(2)$ & $\mathrm{C}(10)$ & $\mathrm{C}(13)$ & 126.2(9) \\
\hline C(15) & $\mathrm{P}(1)$ & $C(14)$ & $103.2(4)$ & $C(15)^{1)}$ & $\mathrm{P}(1)$ & $C(14)$ & $103.2(4)$ \\
\hline$C(15)^{1)}$ & $\mathrm{P}(1)$ & C(15) & $103.8(4)$ & $\mathrm{N}(1)$ & $\mathrm{C}(1)$ & $\mathrm{N}(2)$ & 179.3(8) \\
\hline$C(3)^{1)}$ & $C(2)$ & C(3) & 106.8(9) & $\mathrm{C}(2)$ & $\mathrm{C}(3)$ & $\mathrm{C}(4)$ & $108.4(7)$ \\
\hline C(5) & $\mathrm{C}(2)$ & C(3) & $126.5(5)$ & $C(2)$ & $\mathrm{C}(3)$ & C(6) & 124.8(8) \\
\hline $\mathrm{C}(2)$ & $\mathrm{C}(3)^{1)}$ & $C(4)^{1)}$ & $108.4(7)$ & $\mathrm{C}(5)$ & $\mathrm{C}(2)$ & $C(3)^{1)}$ & $126.5(5)$ \\
\hline $\mathrm{C}(2)$ & $C(3)^{1)}$ & $C(6)^{1)}$ & $124.8(8)$ & $\mathrm{C}(3)$ & $C(4)$ & $C(4)^{1)}$ & 108.1(7) \\
\hline C(6) & C(3) & C(4) & $126.7(7)$ & C(3) & $C(4)$ & C(7) & $124.9(7)$ \\
\hline C(4) & $C(4)^{1)}$ & $C(3)^{1)}$ & 108.1(7) & $\mathrm{C}(7)$ & $\mathrm{C}(4)$ & $C(4)^{1)}$ & 126.8(7) \\
\hline C(4) & $\mathrm{C}(4)^{1)}$ & $\mathrm{C}(7)^{1)}$ & 126.8(7) & $\mathrm{C}(9)$ & $\mathrm{C}(8)$ & $C(8)^{1)}$ & $108.4(6)$ \\
\hline C(8) & $C(8)^{1)}$ & $\mathrm{C}(9)^{1)}$ & $108.4(6)$ & $C(11)$ & $\mathrm{C}(8)$ & $C(8)^{1)}$ & 126.3(7) \\
\hline C(8) & $\mathrm{C}(8)^{1)}$ & $C(11)^{1)}$ & $126.3(7)$ & $C(8)$ & $\mathrm{C}(9)$ & $C(10)$ & $107.0(7)$ \\
\hline $\mathrm{C}(11)$ & C(8) & C(9) & $125.2(7)$ & C(8) & C(9) & $C(12)$ & $126.0(7)$ \\
\hline $\mathrm{C}(9)$ & $C(10)$ & $C(9)^{1)}$ & $108.9(9)$ & $C(12)$ & C(9) & $C(10)$ & $126.9(8)$ \\
\hline C(9) & $\mathrm{C}(10)$ & $\mathrm{C}(13)$ & $125.5(5)$ & $C(10)$ & $\mathrm{C}(9)^{1)}$ & $C(8)^{1)}$ & $107.0(7)$ \\
\hline $\mathrm{C}(10)$ & $C(9)^{1)}$ & $C(12)^{1)}$ & $126.9(8)$ & $\mathrm{C}(13)$ & $C(10)$ & $C(9)^{1)}$ & $125.5(5)$ \\
\hline
\end{tabular}

Angles are in degrees. Symmetry operations: 1$),-\mathrm{X},-\mathrm{Y}+1 / 2, \mathrm{Z}$. 
Table S15. Positional Parameters and B(eq) for $\left[\left\{\mathrm{Cp} * \operatorname{Ir}\left(\mu_{2}-\mathrm{NCN}-N, N\right)\right\}_{2}(\mu\right.$-dppm) $] \cdot 1.5 \mathrm{C}_{6} \mathrm{H}_{6}\left(\mathbf{9} \cdot 1.5 \mathrm{C}_{6} \mathrm{H}_{6}\right)$.

\begin{tabular}{|c|c|c|c|c|}
\hline atom & $\mathrm{x}$ & $\mathrm{y}$ & $\mathrm{z}$ & $\mathrm{B}(\mathrm{eq})$ \\
\hline $\operatorname{Ir}(1)$ & $0.10772(1)$ & $0.47498(1)$ & $0.27106(1)$ & $1.869(4)$ \\
\hline $\operatorname{Ir}(2)$ & $0.40851(1)$ & $0.70784(1)$ & $0.33470(1)$ & $2.121(4)$ \\
\hline $\mathrm{P}(1)$ & $0.1919(1)$ & $0.3908(1)$ & $0.19053(5)$ & $2.01(2)$ \\
\hline $\mathrm{P}(2)$ & $0.4711(1)$ & $0.6056(1)$ & $0.25111(6)$ & $2.21(2)$ \\
\hline $\mathrm{N}(1)$ & $0.2496(3)$ & $0.6600(4)$ & $0.2662(2)$ & $2.35(8)$ \\
\hline $\mathrm{N}(2)$ & $0.2553(5)$ & $0.7590(5)$ & $0.1646(3)$ & $4.7(1)$ \\
\hline $\mathrm{N}(3)$ & $0.2707(4)$ & $0.5170(4)$ & $0.3376(2)$ & 2.42(8) \\
\hline $\mathrm{N}(4)$ & $0.2490(5)$ & $0.4371(5)$ & $0.4467(3)$ & $5.2(1)$ \\
\hline C(1) & $0.2524(5)$ & $0.7100(4)$ & $0.2125(3)$ & $2.8(1)$ \\
\hline $\mathrm{C}(2)$ & $0.2588(5)$ & $0.4762(5)$ & $0.3951(3)$ & $3.0(1)$ \\
\hline C(3) & $-0.0940(4)$ & $0.3236(5)$ & $0.2482(3)$ & $3.2(1)$ \\
\hline C(4) & $-0.0568(4)$ & $0.3560(5)$ & $0.3221(3)$ & $3.2(1)$ \\
\hline C(5) & $-0.0246(5)$ & $0.4878(5)$ & $0.3453(3)$ & $3.2(1)$ \\
\hline C(6) & $-0.0396(5)$ & $0.5354(5)$ & $0.2871(3)$ & $3.2(1)$ \\
\hline$C(7)$ & $-0.0782(5)$ & $0.4362(5)$ & $0.2264(3)$ & $3.2(1)$ \\
\hline C(8) & $-0.1504(6)$ & $0.1918(6)$ & $0.2041(4)$ & $5.7(2)$ \\
\hline $\mathrm{C}(9)$ & $-0.0648(7)$ & $0.2695(7)$ & $0.3708(4)$ & $5.2(2)$ \\
\hline$C(10)$ & $0.0070(6)$ & $0.5577(7)$ & $0.4194(3)$ & $5.4(2)$ \\
\hline $\mathrm{C}(11)$ & $-0.0189(7)$ & $0.6676(7)$ & $0.2884(4)$ & $6.0(2)$ \\
\hline $\mathrm{C}(12)$ & $-0.1165(6)$ & $0.4479(8)$ & $0.1550(3)$ & $5.8(2)$ \\
\hline C(13) & $0.5957(5)$ & $0.8672(5)$ & $0.3851(3)$ & 3.3(1) \\
\hline $\mathrm{C}(14)$ & $0.5305(5)$ & $0.7999(5)$ & $0.4374(3)$ & $3.5(1)$ \\
\hline $\mathrm{C}(15)$ & $0.4205(5)$ & $0.8167(5)$ & $0.4435(3)$ & $3.6(1)$ \\
\hline $\mathrm{C}(16)$ & $0.4170(5)$ & $0.8869(5)$ & $0.3946(3)$ & $3.8(1)$ \\
\hline $\mathrm{C}(17)$ & $0.5239(5)$ & $0.9165(5)$ & $0.3554(3)$ & $3.3(1)$ \\
\hline C(18) & $0.7266(5)$ & $0.8932(6)$ & $0.3700(4)$ & $4.9(1)$ \\
\hline C(19) & $0.5758(7)$ & $0.7437(7)$ & $0.4865(3)$ & $5.7(2)$ \\
\hline C(20) & $0.3323(7)$ & $0.7697(6)$ & 0.4977(3) & $5.8(2)$ \\
\hline $\mathrm{C}(21)$ & $0.3189(7)$ & $0.9264(6)$ & $0.3832(4)$ & $6.4(2)$ \\
\hline $\mathrm{C}(22)$ & $0.5627(7)$ & $1.0021(5)$ & $0.3034(3)$ & $5.3(2)$ \\
\hline C(23) & $0.1071(4)$ & $0.3337(5)$ & $0.1012(2)$ & $2.53(9)$ \\
\hline C(24) & $0.0313(5)$ & $0.2040(5)$ & $0.0717(3)$ & $3.8(1)$ \\
\hline C(25) & $-0.0349(6)$ & $0.1652(7)$ & $0.0061(3)$ & $5.5(2)$ \\
\hline$C(26)$ & $-0.0301(7)$ & $0.2510(8)$ & $-0.0317(3)$ & $5.6(2)$ \\
\hline $\mathrm{C}(27)$ & $0.0457(6)$ & $0.3818(7)$ & $-0.0036(3)$ & $4.7(2)$ \\
\hline C(28) & $0.1122(5)$ & $0.4227(6)$ & $0.0634(3)$ & $3.5(1)$ \\
\hline C(29) & $0.2071(5)$ & $0.2538(4)$ & $0.2046(2)$ & $2.6(1)$ \\
\hline C(30) & $0.1744(7)$ & $0.2061(6)$ & $0.2639(3)$ & $4.6(2)$ \\
\hline $\mathrm{C}(31)$ & 0.1799(9) & $0.0971(7)$ & $0.2719(4)$ & $7.1(2)$ \\
\hline C(32) & $0.2212(8)$ & $0.0379(7)$ & $0.2212(4)$ & $6.3(2)$ \\
\hline C(33) & $0.2559(6)$ & $0.0856(6)$ & $0.1623(4)$ & $4.7(2)$ \\
\hline C(34) & $0.2508(5)$ & $0.1943(5)$ & $0.1548(3)$ & $3.7(1)$ \\
\hline C(35) & $0.3514(4)$ & $0.5069(4)$ & $0.1751(2)$ & $2.30(9)$ \\
\hline C(36) & $0.5264(4)$ & $0.5016(4)$ & $0.2788(3)$ & $2.7(1)$ \\
\hline C(37) & $0.5548(6)$ & $0.5124(5)$ & $0.3489(3)$ & $3.7(1)$ \\
\hline C(38) & $0.6102(6)$ & $0.4473(6)$ & $0.3724(3)$ & $4.5(1)$ \\
\hline C(39) & $0.6347(6)$ & $0.3667(6)$ & $0.3252(4)$ & $4.7(2)$ \\
\hline$C(40)$ & $0.6029(6)$ & $0.3514(6)$ & $0.2551(4)$ & $4.6(2)$ \\
\hline $\mathrm{C}(41)$ & $0.5501(5)$ & $0.4202(6)$ & $0.2311(3)$ & $3.8(1)$ \\
\hline $\mathrm{C}(42)$ & $0.6015(4)$ & $0.7104(5)$ & $0.2062(2)$ & $2.80(9)$ \\
\hline$C(43)$ & $0.7202(5)$ & $0.7188(5)$ & $0.2129(3)$ & 4.1(1) \\
\hline$C(44)$ & $0.8165(5)$ & $0.8014(6)$ & $0.1793(4)$ & $5.0(2)$ \\
\hline$C(45)$ & $0.7952(6)$ & $0.8750(6)$ & $0.1411(4)$ & $5.4(2)$ \\
\hline$C(46)$ & $0.6798(6)$ & $0.8688(5)$ & $0.1342(3)$ & $4.4(1)$ \\
\hline C(47) & $0.5827(5)$ & $0.7872(4)$ & $0.1678(3)$ & $3.3(1)$ \\
\hline
\end{tabular}




\begin{tabular}{|c|c|c|c|c|}
\hline$C(48)$ & $0.592(1)$ & $0.474(1)$ & $0.0217(4)$ & $8.0(3)$ \\
\hline $\mathrm{C}(49)$ & $0.6249(9)$ & $0.594(1)$ & $0.0124(5)$ & $8.1(3)$ \\
\hline $\mathrm{C}(50)$ & $0.530(1)$ & $0.618(1)$ & $-0.0109(5)$ & 8.7(3) \\
\hline $\mathrm{C}(51)$ & $0.0368(8)$ & $-0.0408(8)$ & $0.4405(4)$ & $6.4(2)$ \\
\hline C(52) & $0.0715(6)$ & $0.0874(7)$ & $0.4602(4)$ & $5.2(2)$ \\
\hline C(53) & $0.0361(8)$ & $0.1268(7)$ & $0.5193(4)$ & $6.0(2)$ \\
\hline C(54) & $0.569(2)$ & $1.106(2)$ & $0.049(1)$ & $13.2(6)$ \\
\hline C(55) & $0.479(3)$ & $0.996(3)$ & $0.0652(8)$ & $12.5(6)$ \\
\hline C(56) & $0.413(1)$ & $0.895(2)$ & $0.016(2)$ & 11.8(5) \\
\hline $\mathrm{H}(1)$ & -0.2356 & 0.1639 & 0.1860 & 5.501 \\
\hline $\mathrm{H}(2)$ & -0.1020 & 0.1932 & 0.1665 & 5.503 \\
\hline $\mathrm{H}(3)$ & -0.1495 & 0.1344 & 0.2318 & 5.504 \\
\hline $\mathrm{H}(4)$ & -0.1263 & 0.2625 & 0.4015 & 6.538 \\
\hline $\mathrm{H}(5)$ & -0.0888 & 0.1867 & 0.3440 & 6.544 \\
\hline $\mathrm{H}(6)$ & 0.0159 & 0.3041 & 0.3976 & 6.542 \\
\hline $\mathrm{H}(7)$ & 0.0793 & 0.5582 & 0.4403 & 6.339 \\
\hline $\mathrm{H}(8)$ & 0.0253 & 0.6436 & 0.4210 & 6.339 \\
\hline $\mathrm{H}(9)$ & -0.0633 & 0.5157 & 0.4441 & 6.334 \\
\hline $\mathrm{H}(10)$ & 0.0672 & 0.7278 & 0.3068 & 8.210 \\
\hline $\mathrm{H}(11)$ & -0.0350 & 0.6769 & 0.2425 & 8.202 \\
\hline $\mathrm{H}(12)$ & -0.0746 & 0.6819 & 0.3172 & 8.206 \\
\hline $\mathrm{H}(13)$ & -0.0480 & 0.5180 & 0.1405 & 7.418 \\
\hline $\mathrm{H}(14)$ & -0.1382 & 0.3702 & 0.1233 & 7.408 \\
\hline $\mathrm{H}(15)$ & -0.1872 & 0.4621 & 0.1566 & 7.421 \\
\hline H(16) & 0.7256 & 0.8140 & 0.3550 & 5.146 \\
\hline $\mathrm{H}(17)$ & 0.7498 & 0.9410 & 0.3338 & 5.142 \\
\hline $\mathrm{H}(18)$ & 0.7856 & 0.9405 & 0.4112 & 5.130 \\
\hline H(19) & 0.5119 & 0.7031 & 0.5151 & 6.502 \\
\hline $\mathrm{H}(20)$ & 0.5942 & 0.6823 & 0.4607 & 6.505 \\
\hline $\mathrm{H}(21)$ & 0.6510 & 0.8103 & 0.5152 & 6.501 \\
\hline $\mathrm{H}(22)$ & 0.3802 & 0.8106 & 0.5426 & 5.705 \\
\hline $\mathrm{H}(23)$ & 0.2655 & 0.7892 & 0.4934 & 5.695 \\
\hline $\mathrm{H}(24)$ & 0.2957 & 0.6789 & 0.4917 & 5.696 \\
\hline $\mathrm{H}(25)$ & 0.3166 & 0.9773 & 0.4250 & 7.695 \\
\hline $\mathrm{H}(26)$ & 0.3398 & 0.9747 & 0.3473 & 7.693 \\
\hline $\mathrm{H}(27)$ & 0.2375 & 0.8516 & 0.3695 & 7.701 \\
\hline $\mathrm{H}(28)$ & 0.6362 & 1.0062 & 0.2851 & 5.681 \\
\hline $\mathrm{H}(29)$ & 0.4946 & 0.9669 & 0.2666 & 5.679 \\
\hline $\mathrm{H}(30)$ & 0.5815 & 1.0862 & 0.3256 & 5.683 \\
\hline $\mathrm{H}(31)$ & 0.0253 & 0.1412 & 0.0971 & 4.068 \\
\hline H(32) & -0.0854 & 0.0757 & -0.0133 & 5.591 \\
\hline H(33) & -0.0778 & 0.2225 & -0.0768 & 6.692 \\
\hline H(34) & 0.0523 & 0.4434 & -0.0302 & 6.179 \\
\hline $\mathrm{H}(35)$ & 0.1611 & 0.5121 & 0.0831 & 4.485 \\
\hline $\mathrm{H}(36)$ & 0.1476 & 0.2476 & 0.2998 & 6.080 \\
\hline $\mathrm{H}(37)$ & 0.1553 & 0.0633 & 0.3130 & 10.087 \\
\hline H(38) & 0.2252 & -0.0364 & 0.2274 & 8.374 \\
\hline H(39) & 0.2833 & 0.0443 & 0.1267 & 6.014 \\
\hline $\mathrm{H}(40)$ & 0.2782 & 0.2296 & 0.1144 & 4.573 \\
\hline $\mathrm{H}(41)$ & 0.3833 & 0.4600 & 0.1467 & 2.875 \\
\hline $\mathrm{H}(42)$ & 0.3426 & 0.5640 & 0.1502 & 2.869 \\
\hline $\mathrm{H}(43)$ & 0.5361 & 0.5662 & 0.3819 & 4.755 \\
\hline $\mathrm{H}(44)$ & 0.6313 & 0.4577 & 0.4212 & 5.731 \\
\hline $\mathrm{H}(45)$ & 0.6736 & 0.3220 & 0.3411 & 6.113 \\
\hline $\mathrm{H}(46)$ & 0.6169 & 0.2933 & 0.2225 & 5.985 \\
\hline $\mathrm{H}(47)$ & 0.5305 & 0.4113 & 0.1823 & 4.970 \\
\hline $\mathrm{H}(48)$ & 0.7353 & 0.6682 & 0.2402 & 4.854 \\
\hline
\end{tabular}




\begin{tabular}{lrrrc}
$\mathrm{H}(49)$ & 0.8975 & 0.8065 & 0.1830 & 5.842 \\
$\mathrm{H}(50)$ & 0.8622 & 0.9319 & 0.1187 & 6.294 \\
$\mathrm{H}(51)$ & 0.6658 & 0.9197 & 0.1065 & 5.187 \\
$\mathrm{H}(52)$ & 0.5028 & 0.7844 & 0.1644 & 3.899 \\
$\mathrm{H}(53)$ & 0.6562 & 0.4550 & 0.0357 & 9.792 \\
$\mathrm{H}(54)$ & 0.7112 & 0.6612 & 0.0218 & 10.682 \\
$\mathrm{H}(55)$ & 0.5523 & 0.7004 & -0.0195 & 8.272 \\
$\mathrm{H}(56)$ & 0.0628 & -0.0699 & 0.3993 & 6.129 \\
$\mathrm{H}(57)$ & 0.1200 & 0.1470 & 0.4322 & 1.504 \\
$\mathrm{H}(58)$ & 0.0615 & 0.2154 & 0.5338 & 20.176 \\
$\mathrm{H}(59)$ & 0.6178 & 1.1807 & 0.0849 & 14.823 \\
$\mathrm{H}(60)$ & 0.4627 & 0.9913 & 0.1122 & \\
$\mathrm{H}(61)$ & 0.3504 & 0.8170 & 0.0275 & \\
\hline
\end{tabular}

Table S16. Anisotropic Displacement Parameters for $\left[\left\{\mathrm{Cp} * \operatorname{Ir}\left(\mu_{2}-N C N-N, N\right)\right\}_{2}(\mu-\mathrm{dppm})\right] \cdot 1.5 \mathrm{C}_{6} \mathrm{H}_{6}\left(\mathbf{9} \cdot 1.5 \mathrm{C}_{6} \mathrm{H}_{6}\right)$.

\begin{tabular}{|c|c|c|c|c|c|c|}
\hline atom & U11 & U22 & U33 & U12 & U13 & U23 \\
\hline $\operatorname{Ir}(1)$ & $0.02362(9)$ & $0.02677(9)$ & $0.02012(9)$ & $0.01161(7)$ & $0.00317(6)$ & $0.00627(6)$ \\
\hline $\operatorname{Ir}(2)$ & $0.0268(1)$ & $0.0252(1)$ & $0.0233(1)$ & $0.00962(7)$ & $0.00159(7)$ & $0.00225(7)$ \\
\hline $\mathrm{P}(1)$ & $0.0278(5)$ & $0.0268(5)$ & $0.0201(5)$ & $0.0126(5)$ & $0.0021(4)$ & $0.0042(4)$ \\
\hline $\mathrm{P}(2)$ & $0.0265(6)$ & $0.0299(6)$ & $0.0270(6)$ & $0.0131(5)$ & $0.0040(4)$ & $0.0063(5)$ \\
\hline $\mathrm{N}(1)$ & $0.030(2)$ & $0.033(2)$ & $0.028(2)$ & $0.015(2)$ & $0.005(2)$ & $0.012(2)$ \\
\hline $\mathrm{N}(2)$ & $0.077(4)$ & $0.061(3)$ & $0.048(3)$ & $0.034(3)$ & $0.008(2)$ & $0.031(2)$ \\
\hline $\mathrm{N}(3)$ & $0.032(2)$ & $0.032(2)$ & $0.024(2)$ & $0.013(2)$ & $0.001(2)$ & $0.008(2)$ \\
\hline $\mathrm{N}(4)$ & $0.068(4)$ & $0.071(4)$ & $0.039(3)$ & $0.014(3)$ & $-0.012(2)$ & $0.029(2)$ \\
\hline $\mathrm{C}(1)$ & $0.037(3)$ & $0.028(2)$ & $0.039(3)$ & $0.016(2)$ & $0.011(2)$ & $0.004(2)$ \\
\hline $\mathrm{C}(2)$ & $0.037(3)$ & $0.034(3)$ & $0.031(2)$ & $0.009(2)$ & $-0.005(2)$ & $0.006(2)$ \\
\hline C(3) & $0.027(2)$ & $0.043(3)$ & $0.039(3)$ & $0.010(2)$ & $0.010(2)$ & $0.003(2)$ \\
\hline $\mathrm{C}(4)$ & $0.030(2)$ & $0.047(3)$ & $0.042(3)$ & $0.014(2)$ & $0.019(2)$ & $0.022(2)$ \\
\hline C(5) & $0.037(3)$ & $0.056(3)$ & $0.034(3)$ & $0.025(2)$ & $0.009(2)$ & $0.010(2)$ \\
\hline C(6) & $0.033(3)$ & $0.049(3)$ & $0.045(3)$ & $0.025(2)$ & $0.006(2)$ & $0.010(2)$ \\
\hline C(7) & $0.028(2)$ & 0.061(3) & $0.033(3)$ & $0.020(2)$ & $0.004(2)$ & $0.016(2)$ \\
\hline $\mathrm{C}(8)$ & $0.044(3)$ & $0.051(4)$ & $0.080(5)$ & $-0.003(3)$ & $0.017(3)$ & $-0.014(3)$ \\
\hline $\mathrm{C}(9)$ & $0.065(4)$ & $0.071(4)$ & $0.071(4)$ & $0.033(4)$ & $0.034(3)$ & $0.040(4)$ \\
\hline $\mathrm{C}(10)$ & $0.062(4)$ & $0.099(5)$ & $0.039(3)$ & $0.042(4)$ & $0.014(3)$ & $-0.003(3)$ \\
\hline $\mathrm{C}(11)$ & $0.070(5)$ & $0.080(5)$ & $0.110(6)$ & $0.058(4)$ & $0.017(4)$ & $0.030(4)$ \\
\hline $\mathrm{C}(12)$ & $0.055(4)$ & $0.135(7)$ & $0.045(3)$ & $0.052(4)$ & $0.006(3)$ & $0.038(4)$ \\
\hline C(13) & $0.036(3)$ & $0.034(3)$ & $0.038(3)$ & $0.007(2)$ & $-0.007(2)$ & $-0.005(2)$ \\
\hline$C(14)$ & $0.044(3)$ & $0.040(3)$ & $0.029(2)$ & $0.011(2)$ & $-0.010(2)$ & $-0.006(2)$ \\
\hline C(15) & $0.044(3)$ & $0.038(3)$ & $0.029(2)$ & $0.007(2)$ & $0.002(2)$ & $-0.014(2)$ \\
\hline $\mathrm{C}(16)$ & $0.052(3)$ & $0.036(3)$ & $0.046(3)$ & $0.020(3)$ & $-0.004(2)$ & $-0.013(2)$ \\
\hline$C(17)$ & $0.049(3)$ & $0.029(2)$ & $0.037(3)$ & $0.014(2)$ & $-0.002(2)$ & $0.001(2)$ \\
\hline C(18) & $0.032(3)$ & $0.059(4)$ & $0.071(4)$ & $0.007(3)$ & $-0.004(3)$ & $0.004(3)$ \\
\hline C(19) & $0.094(5)$ & $0.071(4)$ & $0.041(3)$ & $0.040(4)$ & $-0.027(3)$ & $-0.005(3)$ \\
\hline C(20) & $0.075(4)$ & $0.065(4)$ & $0.040(3)$ & $0.010(3)$ & $0.018(3)$ & $-0.013(3)$ \\
\hline $\mathrm{C}(21)$ & $0.089(5)$ & $0.055(4)$ & $0.099(6)$ & $0.049(4)$ & $-0.015(4)$ & $-0.018(4)$ \\
\hline $\mathrm{C}(22)$ & $0.090(5)$ & $0.033(3)$ & $0.057(4)$ & $0.014(3)$ & $0.009(3)$ & 0.011(3) \\
\hline $\mathrm{C}(23)$ & $0.034(2)$ & $0.042(3)$ & $0.021(2)$ & $0.020(2)$ & $0.002(2)$ & $0.002(2)$ \\
\hline$C(24)$ & $0.046(3)$ & $0.047(3)$ & $0.035(3)$ & $0.012(3)$ & $-0.007(2)$ & $0.003(2)$ \\
\hline$C(25)$ & $0.059(4)$ & $0.072(4)$ & $0.046(3)$ & $0.017(3)$ & $-0.019(3)$ & $-0.014(3)$ \\
\hline$C(26)$ & $0.070(4)$ & $0.111(6)$ & $0.031(3)$ & $0.048(4)$ & $-0.016(3)$ & $0.003(4)$ \\
\hline$C(27)$ & $0.074(4)$ & $0.094(5)$ & $0.028(3)$ & $0.055(4)$ & $0.003(3)$ & $0.015(3)$ \\
\hline C(28) & $0.055(3)$ & $0.058(3)$ & $0.029(2)$ & $0.033(3)$ & $0.003(2)$ & $0.010(2)$ \\
\hline C(29) & $0.040(3)$ & $0.029(2)$ & $0.034(2)$ & $0.020(2)$ & $-0.002(2)$ & $0.004(2)$ \\
\hline $\mathrm{C}(30)$ & $0.107(5)$ & $0.048(3)$ & $0.038(3)$ & $0.049(4)$ & $0.017(3)$ & $0.016(3)$ \\
\hline C(31) & $0.171(9)$ & $0.084(5)$ & $0.065(5)$ & $0.093(6)$ & $0.037(5)$ & $0.038(4)$ \\
\hline C(32) & $0.130(7)$ & $0.058(4)$ & $0.078(5)$ & $0.066(5)$ & $0.007(5)$ & $0.015(4)$ \\
\hline
\end{tabular}




\begin{tabular}{lrlllrr} 
C(33) & $0.073(4)$ & $0.054(4)$ & $0.063(4)$ & $0.045(3)$ & $0.003(3)$ & $-0.001(3)$ \\
C(34) & $0.053(3)$ & $0.042(3)$ & $0.050(3)$ & $0.026(3)$ & $0.006(3)$ & $0.010(2)$ \\
C(35) & $0.031(2)$ & $0.034(2)$ & $0.026(2)$ & $0.019(2)$ & $0.005(2)$ & $0.006(2)$ \\
C(36) & $0.030(2)$ & $0.033(2)$ & $0.037(3)$ & $0.015(2)$ & $0.002(2)$ & $0.009(2)$ \\
C(37) & $0.063(4)$ & $0.054(3)$ & $0.034(3)$ & $0.038(3)$ & $0.003(2)$ & $0.009(2)$ \\
C(38) & $0.064(4)$ & $0.070(4)$ & $0.047(3)$ & $0.036(3)$ & $0.004(3)$ & $0.024(3)$ \\
C(39) & $0.054(4)$ & $0.065(4)$ & $0.075(4)$ & $0.038(3)$ & $0.009(3)$ & $0.028(3)$ \\
C(40) & $0.057(4)$ & $0.055(4)$ & $0.078(5)$ & $0.039(3)$ & $0.012(3)$ & $0.008(3)$ \\
C(41) & $0.048(3)$ & $0.060(3)$ & $0.050(3)$ & $0.036(3)$ & $0.006(3)$ & $0.010(3)$ \\
C(42) & $0.031(2)$ & $0.034(2)$ & $0.033(2)$ & $0.009(2)$ & $0.008(2)$ & $0.008(2)$ \\
C(43) & $0.035(3)$ & $0.053(3)$ & $0.065(4)$ & $0.018(3)$ & $0.013(3)$ & $0.020(3)$ \\
C(44) & $0.032(3)$ & $0.061(4)$ & $0.091(5)$ & $0.015(3)$ & $0.022(3)$ & $0.026(4)$ \\
C(45) & $0.060(4)$ & $0.051(4)$ & $0.089(5)$ & $0.018(3)$ & $0.043(4)$ & $0.023(3)$ \\
C(46) & $0.062(4)$ & $0.034(3)$ & $0.068(4)$ & $0.018(3)$ & $0.025(3)$ & $0.022(3)$ \\
C(47) & $0.044(3)$ & $0.037(3)$ & $0.043(3)$ & $0.018(2)$ & $0.012(2)$ & $0.011(2)$ \\
C(48) & $0.095(8)$ & $0.152(9)$ & $0.063(5)$ & $0.071(7)$ & $0.019(5)$ & $0.004(6)$ \\
C(49) & $0.084(7)$ & $0.14(1)$ & $0.063(5)$ & $0.048(7)$ & $0.014(4)$ & $-0.010(6)$ \\
C(50) & $0.135(9)$ & $0.126(9)$ & $0.078(6)$ & $0.070(9)$ & $0.052(6)$ & $0.009(6)$ \\
C(51) & $0.089(6)$ & $0.095(6)$ & $0.078(5)$ & $0.064(5)$ & $0.021(4)$ & $0.003(4)$ \\
C(52) & $0.047(4)$ & $0.077(5)$ & $0.070(4)$ & $0.028(3)$ & $0.005(3)$ & $0.020(4)$ \\
C(53) & $0.085(5)$ & $0.064(4)$ & $0.089(5)$ & $0.047(4)$ & $-0.001(4)$ & $0.006(4)$ \\
C(54) & $0.24(2)$ & $0.13(1)$ & $0.13(1)$ & $0.11(1)$ & $-0.09(1)$ & $-0.044(9)$ \\
C(55) & $0.24(2)$ & $0.32(2)$ & $0.074(8)$ & $0.25(2)$ & $0.08(1)$ & $0.09(1)$ \\
C(56) & $0.061(6)$ & $0.14(1)$ & $0.27(2)$ & $0.042(7)$ & $0.02(1)$ & $0.12(1)$ \\
\hline
\end{tabular}


Table S17. Intramolecular Distances Involving the Nonhydrogen Atoms

for $\left[\left\{\mathrm{Cp} * \operatorname{Ir}\left(\mu_{2}-\mathrm{NCN}-N, N\right)\right\}_{2}(\mu-\mathrm{dppm})\right] \cdot 1.5 \mathrm{C}_{6} \mathrm{H}_{6}\left(\mathbf{9} \cdot 1.5 \mathrm{C}_{6} \mathrm{H}_{6}\right)$.

\begin{tabular}{|c|c|c|c|c|c|}
\hline atom & atom & distance & atom & atom & distance \\
\hline $\operatorname{Ir}(1)$ & $\mathrm{P}(1)$ & $2.264(1)$ & $\operatorname{Ir}(1)$ & $\mathrm{N}(1)$ & $2.082(3)$ \\
\hline $\operatorname{Ir}(1)$ & $\mathrm{N}(3)$ & $2.117(4)$ & $\operatorname{Ir}(1)$ & C(3) & $2.202(4)$ \\
\hline $\operatorname{Ir}(1)$ & C(4) & $2.202(5)$ & $\operatorname{Ir}(1)$ & $C(5)$ & $2.234(6)$ \\
\hline $\operatorname{Ir}(1)$ & C(6) & $2.241(7)$ & $\operatorname{Ir}(1)$ & $\mathrm{C}(7)$ & $2.181(6)$ \\
\hline $\operatorname{Ir}(2)$ & $\mathrm{P}(2)$ & 2.256(1) & $\operatorname{Ir}(2)$ & $\mathrm{N}(1)$ & $2.080(4)$ \\
\hline $\operatorname{Ir}(2)$ & N(3) & 2.108(3) & $\operatorname{Ir}(2)$ & C(13) & $2.198(4)$ \\
\hline $\operatorname{Ir}(2)$ & $C(14)$ & $2.221(5)$ & $\operatorname{Ir}(2)$ & $C(15)$ & $2.270(5)$ \\
\hline $\operatorname{Ir}(2)$ & C(16) & $2.243(6)$ & $\operatorname{Ir}(2)$ & $\mathrm{C}(17)$ & $2.172(5)$ \\
\hline $\mathrm{P}(1)$ & $C(23)$ & $1.838(4)$ & $\mathrm{P}(1)$ & C(29) & $1.817(6)$ \\
\hline $\mathrm{P}(1)$ & C(35) & $1.827(4)$ & $\mathrm{P}(2)$ & C(35) & $1.827(4)$ \\
\hline $\mathrm{P}(2)$ & $C(36)$ & $1.827(7)$ & $P(2)$ & $\mathrm{C}(42)$ & $1.838(5)$ \\
\hline $\mathrm{N}(1)$ & $\mathrm{C}(1)$ & $1.285(7)$ & $\mathrm{N}(2)$ & $\mathrm{C}(1)$ & $1.180(8)$ \\
\hline$N(3)$ & $C(2)$ & $1.283(7)$ & $\mathrm{N}(4)$ & $C(2)$ & $1.168(8)$ \\
\hline C(3) & C(4) & $1.430(7)$ & $\mathrm{C}(3)$ & $C(7)$ & 1.421(9) \\
\hline C(3) & C(8) & $1.489(8)$ & $C(4)$ & $\mathrm{C}(5)$ & $1.434(8)$ \\
\hline C(4) & C(9) & $1.50(1)$ & $\mathrm{C}(5)$ & $C(6)$ & 1.398(9) \\
\hline C(5) & C(10) & $1.486(7)$ & $C(6)$ & $\mathrm{C}(7)$ & $1.424(7)$ \\
\hline$C(6)$ & $\mathrm{C}(11)$ & $1.49(1)$ & $C(7)$ & $\mathrm{C}(12)$ & $1.503(9)$ \\
\hline C(13) & $\mathrm{C}(14)$ & $1.425(7)$ & $\mathrm{C}(13)$ & $C(17)$ & $1.42(1)$ \\
\hline C(13) & C(18) & $1.506(9)$ & $\mathrm{C}(14)$ & C(15) & 1.44(1) \\
\hline $\mathrm{C}(14)$ & C(19) & $1.49(1)$ & $C(15)$ & $C(16)$ & $1.390(9)$ \\
\hline $\mathrm{C}(15)$ & $C(20)$ & $1.503(8)$ & $\mathrm{C}(16)$ & $\mathrm{C}(17)$ & $1.445(8)$ \\
\hline$C(16)$ & $\mathrm{C}(21)$ & $1.50(1)$ & $C(17)$ & $C(22)$ & $1.493(9)$ \\
\hline C(23) & $C(24)$ & $1.385(6)$ & C(23) & $C(28)$ & $1.382(9)$ \\
\hline $\mathrm{C}(24)$ & C(23) & $1.385(6)$ & C(24) & C(25) & $1.370(8)$ \\
\hline $\mathrm{C}(25)$ & $C(24)$ & $1.370(8)$ & C(25) & $C(26)$ & $1.35(1)$ \\
\hline $\mathrm{C}(26)$ & $\mathrm{C}(27)$ & $1.39(1)$ & $\mathrm{C}(27)$ & C(28) & $1.392(7)$ \\
\hline C(29) & C(30) & $1.362(8)$ & C(29) & C(34) & $1.384(9)$ \\
\hline C(30) & C(29) & $1.362(8)$ & C(30) & C(31) & $1.39(1)$ \\
\hline C(31) & C(30) & $1.39(1)$ & $\mathrm{C}(31)$ & C(32) & 1.37(1) \\
\hline C(32) & C(33) & $1.36(1)$ & C(33) & C(34) & $1.38(1)$ \\
\hline $\mathrm{C}(36)$ & C(37) & $1.370(7)$ & C(36) & C(41) & $1.381(9)$ \\
\hline C(37) & C(38) & $1.37(1)$ & C(38) & C(39) & $1.38(1)$ \\
\hline C(39) & $C(40)$ & $1.37(1)$ & $\mathrm{C}(40)$ & $\mathrm{C}(41)$ & 1.39(1) \\
\hline $\mathrm{C}(42)$ & $C(43)$ & 1.389(9) & $C(42)$ & $\mathrm{C}(47)$ & $1.379(9)$ \\
\hline C(43) & $C(44)$ & $1.387(8)$ & C(44) & $C(45)$ & $1.36(1)$ \\
\hline C(45) & $C(46)$ & $1.36(1)$ & $\mathrm{C}(46)$ & C(47) & $1.387(7)$ \\
\hline C(48) & C(49) & $1.36(2)$ & C(48) & $C(50)^{1)}$ & $1.33(2)$ \\
\hline C(49) & C(50) & $1.39(2)$ & $\mathrm{C}(50)$ & $C(48)^{1)}$ & $1.33(2)$ \\
\hline $\mathrm{C}(51)$ & C(52) & $1.38(1)$ & $\mathrm{C}(51)$ & $\mathrm{C}(53)^{2)}$ & $1.38(1)$ \\
\hline $\mathrm{C}(52)$ & $\mathrm{C}(53)$ & $1.34(1)$ & $\mathrm{C}(53)$ & $\mathrm{C}(51)^{2)}$ & $1.38(1)$ \\
\hline $\mathrm{C}(54)$ & $C(55)$ & $1.35(4)$ & C(54) & $C(56)^{3)}$ & $1.29(4)$ \\
\hline C(55) & $C(56)$ & $1.30(3)$ & C(56) & $C(54)^{3)}$ & $1.29(4)$ \\
\hline
\end{tabular}

Distances are in angstroms.

Symmetry operations: 1 ), $-\mathrm{X}+1,-\mathrm{Y}+1,-\mathrm{Z}$; 2), $-\mathrm{X},-\mathrm{Y},-\mathrm{Z}+1$; 3), $-\mathrm{X}+1,-\mathrm{Y}+2,-\mathrm{Z}$. 
Table S18. Intramolecular Bond Angles Involving the Nonhydrogen Atoms for $\left[\left\{\mathrm{Cp} * \operatorname{Ir}\left(\mu_{2}-\mathrm{NCN}-N, N\right)\right\}_{2}(\mu-\mathrm{dppm})\right] \cdot 1.5 \mathrm{C}_{6} \mathrm{H}_{6}\left(\mathbf{9} \cdot 1.5 \mathrm{C}_{6} \mathrm{H}_{6}\right)$.

\begin{tabular}{|c|c|c|c|c|c|c|c|}
\hline atom & atom & atom & angle & atom & atom & atom & angle \\
\hline $\mathrm{N}(1)$ & $\operatorname{Ir}(1)$ & $\mathrm{P}(1)$ & $90.0(1)$ & $\mathrm{N}(3)$ & $\operatorname{Ir}(1)$ & $\mathrm{P}(1)$ & 83.3(1) \\
\hline $\mathrm{C}(3)$ & $\operatorname{Ir}(1)$ & $\mathrm{P}(1)$ & 99.9(1) & $C(4)$ & $\operatorname{Ir}(1)$ & $\mathrm{P}(1)$ & 123.2(2) \\
\hline C(5) & $\operatorname{Ir}(1)$ & $\mathrm{P}(1)$ & $160.6(1)$ & $\mathrm{C}(6)$ & $\operatorname{Ir}(1)$ & $\mathrm{P}(1)$ & 144.8(1) \\
\hline C(7) & $\operatorname{Ir}(1)$ & $\mathrm{P}(1)$ & 109.6(1) & $\operatorname{Ir}(1)$ & $\mathrm{P}(1)$ & C(23) & $115.3(2)$ \\
\hline $\operatorname{Ir}(1)$ & $\mathrm{P}(1)$ & C(29) & $118.6(2)$ & $\operatorname{Ir}(1)$ & $\mathrm{P}(1)$ & C(35) & 113.9(2) \\
\hline $\operatorname{Ir}(1)$ & $\mathrm{N}(1)$ & $\operatorname{Ir}(2)$ & 108.3(2) & $\mathrm{N}(3)$ & $\operatorname{Ir}(1)$ & $\mathrm{N}(1)$ & 72.7(2) \\
\hline $\operatorname{Ir}(1)$ & $\mathrm{N}(1)$ & $\mathrm{C}(1)$ & 122.2(3) & $\mathrm{C}(3)$ & $\operatorname{Ir}(1)$ & $\mathrm{N}(1)$ & 149.1(2) \\
\hline C(4) & $\operatorname{Ir}(1)$ & $\mathrm{N}(1)$ & 146.7(2) & $\mathrm{C}(5)$ & $\operatorname{Ir}(1)$ & $\mathrm{N}(1)$ & 109.3(2) \\
\hline C(6) & $\operatorname{Ir}(1)$ & $\mathrm{N}(1)$ & 93.1(2) & $C(7)$ & $\operatorname{Ir}(1)$ & $\mathrm{N}(1)$ & $111.3(2)$ \\
\hline $\operatorname{Ir}(1)$ & $\mathrm{N}(3)$ & $\operatorname{Ir}(2)$ & $105.9(2)$ & $\operatorname{Ir}(1)$ & $\mathrm{N}(3)$ & $C(2)$ & 120.4(3) \\
\hline C(3) & $\operatorname{Ir}(1)$ & $\mathrm{N}(3)$ & $137.2(2)$ & $C(4)$ & $\operatorname{Ir}(1)$ & $\mathrm{N}(3)$ & $105.6(2)$ \\
\hline C(5) & $\operatorname{Ir}(1)$ & $\mathrm{N}(3)$ & $103.7(2)$ & $\mathrm{C}(6)$ & $\operatorname{Ir}(1)$ & $\mathrm{N}(3)$ & $131.0(2)$ \\
\hline C(7) & $\operatorname{Ir}(1)$ & $\mathrm{N}(3)$ & 166.1(2) & $\operatorname{Ir}(1)$ & $\mathrm{C}(3)$ & $C(4)$ & 71.1(2) \\
\hline C(4) & $\operatorname{Ir}(1)$ & C(3) & $37.9(2)$ & $\mathrm{C}(5)$ & $\operatorname{Ir}(1)$ & $\mathrm{C}(3)$ & $62.6(2)$ \\
\hline$C(6)$ & $\operatorname{Ir}(1)$ & C(3) & $62.4(2)$ & $\operatorname{Ir}(1)$ & $\mathrm{C}(3)$ & $C(7)$ & $70.2(3)$ \\
\hline C(7) & $\operatorname{Ir}(1)$ & C(3) & $37.8(2)$ & $\operatorname{Ir}(1)$ & $\mathrm{C}(3)$ & $\mathrm{C}(8)$ & $128.4(4)$ \\
\hline $\operatorname{Ir}(1)$ & C(4) & C(3) & 71.1(3) & $\operatorname{Ir}(1)$ & $C(4)$ & $C(5)$ & 72.4(3) \\
\hline C(5) & $\operatorname{Ir}(1)$ & C(4) & $37.7(2)$ & C(6) & $\operatorname{Ir}(1)$ & $C(4)$ & 62.3(2) \\
\hline C(7) & $\operatorname{Ir}(1)$ & C(4) & $63.4(2)$ & $\operatorname{Ir}(1)$ & $C(4)$ & C(9) & $128.0(5)$ \\
\hline $\operatorname{Ir}(1)$ & C(5) & C(4) & 69.9(3) & $\operatorname{Ir}(1)$ & $\mathrm{C}(5)$ & $C(6)$ & 72.1(3) \\
\hline C(6) & $\operatorname{Ir}(1)$ & C(5) & $36.4(2)$ & $C(7)$ & $\operatorname{Ir}(1)$ & $C(5)$ & $62.5(2)$ \\
\hline $\operatorname{Ir}(1)$ & C(5) & C(10) & $128.4(4)$ & $\operatorname{Ir}(1)$ & C(6) & $\mathrm{C}(5)$ & $71.5(4)$ \\
\hline $\operatorname{Ir}(1)$ & C(6) & $\mathrm{C}(7)$ & $68.9(4)$ & $C(7)$ & $\operatorname{Ir}(1)$ & $C(6)$ & $37.5(2)$ \\
\hline $\operatorname{Ir}(1)$ & C(6) & $\mathrm{C}(11)$ & $125.4(4)$ & $\operatorname{Ir}(1)$ & $C(7)$ & C(3) & 71.9(3) \\
\hline $\operatorname{Ir}(1)$ & C(7) & $C(6)$ & 73.6(3) & $\operatorname{Ir}(1)$ & $C(7)$ & $\mathrm{C}(12)$ & $128.4(4)$ \\
\hline $\mathrm{N}(1)$ & $\operatorname{Ir}(2)$ & $\mathrm{P}(2)$ & 89.7(1) & N(3) & $\operatorname{Ir}(2)$ & $P(2)$ & 81.9(1) \\
\hline $\operatorname{Ir}(2)$ & $\mathrm{P}(2)$ & C(35) & 114.7(2) & $\mathrm{C}(13)$ & $\operatorname{Ir}(2)$ & $\mathrm{P}(2)$ & $99.6(2)$ \\
\hline$C(14)$ & $\operatorname{Ir}(2)$ & $P(2)$ & $117.8(2)$ & $C(15)$ & $\operatorname{Ir}(2)$ & $\mathrm{P}(2)$ & $155.2(2)$ \\
\hline$C(16)$ & $\operatorname{Ir}(2)$ & $\mathrm{P}(2)$ & 151.7(1) & $\mathrm{C}(17)$ & $\operatorname{Ir}(2)$ & $\mathrm{P}(2)$ & 114.2(2) \\
\hline $\operatorname{Ir}(2)$ & $\mathrm{P}(2)$ & C(36) & $116.5(2)$ & $\operatorname{Ir}(2)$ & $\mathrm{P}(2)$ & $C(42)$ & $115.6(2)$ \\
\hline N(3) & $\operatorname{Ir}(2)$ & $\mathrm{N}(1)$ & $72.9(2)$ & $\operatorname{Ir}(2)$ & $\mathrm{N}(1)$ & $\mathrm{C}(1)$ & 124.8(3) \\
\hline C(13) & $\operatorname{Ir}(2)$ & $\mathrm{N}(1)$ & $143.8(2)$ & $C(14)$ & $\operatorname{Ir}(2)$ & $\mathrm{N}(1)$ & $152.5(2)$ \\
\hline C(15) & $\operatorname{Ir}(2)$ & $\mathrm{N}(1)$ & $115.0(2)$ & $C(16)$ & $\operatorname{Ir}(2)$ & $\mathrm{N}(1)$ & 93.9(2) \\
\hline $\mathrm{C}(17)$ & $\operatorname{Ir}(2)$ & $\mathrm{N}(1)$ & $106.4(2)$ & $\operatorname{Ir}(2)$ & $\mathrm{N}(3)$ & $\mathrm{C}(2)$ & 120.5(3) \\
\hline C(13) & $\operatorname{Ir}(2)$ & $\mathrm{N}(3)$ & $142.8(2)$ & $C(14)$ & $\operatorname{Ir}(2)$ & $\mathrm{N}(3)$ & $109.0(2)$ \\
\hline$C(15)$ & $\operatorname{Ir}(2)$ & $N(3)$ & 102.6(2) & $C(16)$ & $\operatorname{Ir}(2)$ & $\mathrm{N}(3)$ & $125.9(2)$ \\
\hline $\mathrm{C}(17)$ & $\operatorname{Ir}(2)$ & N(3) & $163.9(2)$ & $\operatorname{Ir}(2)$ & C(13) & C(14) & $72.0(2)$ \\
\hline $\mathrm{C}(14)$ & $\operatorname{Ir}(2)$ & C(13) & $37.6(2)$ & $C(15)$ & $\operatorname{Ir}(2)$ & $\mathrm{C}(13)$ & 61.9(2) \\
\hline C(16) & $\operatorname{Ir}(2)$ & $C(13)$ & $62.2(2)$ & $\operatorname{Ir}(2)$ & C(13) & C(17) & $70.0(2)$ \\
\hline C(17) & $\operatorname{Ir}(2)$ & $C(13)$ & $38.0(2)$ & $\operatorname{Ir}(2)$ & $C(13)$ & C(18) & 129.2(4) \\
\hline $\operatorname{Ir}(2)$ & $C(14)$ & C(13) & 70.3(3) & $\operatorname{Ir}(2)$ & $C(14)$ & $C(15)$ & 73.1(3) \\
\hline$C(15)$ & $\operatorname{Ir}(2)$ & $\mathrm{C}(14)$ & $37.5(2)$ & $C(16)$ & $\operatorname{Ir}(2)$ & $C(14)$ & $62.1(2)$ \\
\hline C(17) & $\operatorname{Ir}(2)$ & $\mathrm{C}(14)$ & 63.8(2) & $\operatorname{Ir}(2)$ & $C(14)$ & $C(19)$ & 130.1(4) \\
\hline $\operatorname{Ir}(2)$ & C(15) & $\mathrm{C}(14)$ & 69.4(3) & $\operatorname{Ir}(2)$ & C(15) & $C(16)$ & $71.0(3)$ \\
\hline$C(16)$ & $\operatorname{Ir}(2)$ & $\mathrm{C}(15)$ & $35.9(2)$ & $C(17)$ & $\operatorname{Ir}(2)$ & $C(15)$ & $62.6(2)$ \\
\hline $\operatorname{Ir}(2)$ & $\mathrm{C}(15)$ & $C(20)$ & 127.9(3) & $\operatorname{Ir}(2)$ & $C(16)$ & C(15) & $73.2(4)$ \\
\hline $\operatorname{Ir}(2)$ & $C(16)$ & $\mathrm{C}(17)$ & 68.3(3) & $C(17)$ & $\operatorname{Ir}(2)$ & $C(16)$ & $38.2(2)$ \\
\hline $\operatorname{Ir}(2)$ & $C(16)$ & $\mathrm{C}(21)$ & $124.5(4)$ & $\operatorname{Ir}(2)$ & C(17) & $C(13)$ & 72.0(3) \\
\hline $\operatorname{Ir}(2)$ & $\mathrm{C}(17)$ & $\mathrm{C}(16)$ & 73.6(3) & $\operatorname{Ir}(2)$ & C(17) & $\mathrm{C}(22)$ & 127.6(3) \\
\hline C(29) & $\mathrm{P}(1)$ & C(23) & 102.1(2) & $C(35)$ & $\mathrm{P}(1)$ & $\mathrm{C}(23)$ & $100.6(2)$ \\
\hline $\mathrm{P}(1)$ & C(23) & $C(24)$ & $122.5(4)$ & $P(1)$ & C(23) & $\mathrm{C}(28)$ & 119.1(3) \\
\hline C(35) & $\mathrm{P}(1)$ & C(29) & 103.9(3) & $\mathrm{P}(1)$ & C(29) & C(30) & $121.0(5)$ \\
\hline $\mathrm{P}(1)$ & C(29) & C(34) & $120.6(4)$ & $\mathrm{P}(1)$ & C(35) & $P(2)$ & $118.0(2)$ \\
\hline C(36) & $P(2)$ & C(35) & $105.7(2)$ & $C(42)$ & $P(2)$ & $C(35)$ & 99.1(2) \\
\hline
\end{tabular}




\begin{tabular}{|c|c|c|c|c|c|c|c|}
\hline$C(42)$ & $\mathrm{P}(2)$ & C(36) & 103.2(3) & $\mathrm{P}(2)$ & C(36) & C(37) & $118.7(5)$ \\
\hline $\mathrm{P}(2)$ & C(36) & $C(41)$ & $121.8(4)$ & $\mathrm{P}(2)$ & $C(42)$ & $C(43)$ & $121.7(5)$ \\
\hline $\mathrm{P}(2)$ & $C(42)$ & $\mathrm{C}(47)$ & 119.1(4) & $\mathrm{N}(1)$ & $\mathrm{C}(1)$ & $\mathrm{N}(2)$ & 178.1(6) \\
\hline $\mathrm{N}(3)$ & $\mathrm{C}(2)$ & $\mathrm{N}(4)$ & $178.4(7)$ & C(3) & $C(4)$ & $\mathrm{C}(5)$ & $107.2(5)$ \\
\hline C(7) & $\mathrm{C}(3)$ & $C(4)$ & $107.8(4)$ & $\mathrm{C}(8)$ & $\mathrm{C}(3)$ & $C(4)$ & $125.2(6)$ \\
\hline C(3) & $\mathrm{C}(4)$ & $\mathrm{C}(9)$ & $128.7(5)$ & $\mathrm{C}(3)$ & $\mathrm{C}(7)$ & $\mathrm{C}(6)$ & $107.9(5)$ \\
\hline C(8) & C(3) & $\mathrm{C}(7)$ & $126.8(5)$ & $\mathrm{C}(3)$ & $C(7)$ & $\mathrm{C}(12)$ & $127.9(5)$ \\
\hline C(4) & $\mathrm{C}(5)$ & $\mathrm{C}(6)$ & $108.5(4)$ & $\mathrm{C}(9)$ & $C(4)$ & $C(5)$ & $123.6(5)$ \\
\hline C(4) & $\mathrm{C}(5)$ & $\mathrm{C}(10)$ & $125.5(6)$ & $\mathrm{C}(5)$ & C(6) & $\mathrm{C}(7)$ & $108.4(5)$ \\
\hline $\mathrm{C}(10)$ & $\mathrm{C}(5)$ & $C(6)$ & $125.8(6)$ & $C(5)$ & C(6) & $\mathrm{C}(11)$ & $125.9(5)$ \\
\hline $\mathrm{C}(11)$ & C(6) & $\mathrm{C}(7)$ & $125.7(6)$ & $\mathrm{C}(6)$ & $\mathrm{C}(7)$ & $C(12)$ & 123.4(7) \\
\hline C(13) & $C(14)$ & $C(15)$ & $106.6(6)$ & $\mathrm{C}(17)$ & C(13) & $C(14)$ & $109.3(5)$ \\
\hline C(18) & C(13) & $\mathrm{C}(14)$ & $124.4(6)$ & $\mathrm{C}(13)$ & $C(14)$ & C(19) & 127.9(6) \\
\hline C(13) & $\mathrm{C}(17)$ & $C(16)$ & 106.3(5) & C(18) & $\mathrm{C}(13)$ & $\mathrm{C}(17)$ & $126.0(5)$ \\
\hline C(13) & $\mathrm{C}(17)$ & $\mathrm{C}(22)$ & $126.9(6)$ & $C(14)$ & $C(15)$ & $C(16)$ & 108.6(5) \\
\hline C(19) & $C(14)$ & $C(15)$ & $124.6(5)$ & $C(14)$ & $C(15)$ & $C(20)$ & $123.2(6)$ \\
\hline C(15) & $C(16)$ & $C(17)$ & 109.1(6) & C(20) & C(15) & $C(16)$ & 128.1(7) \\
\hline C(15) & $C(16)$ & $C(21)$ & $126.3(6)$ & $\mathrm{C}(21)$ & $C(16)$ & $C(17)$ & $124.6(6)$ \\
\hline $\mathrm{C}(16)$ & $\mathrm{C}(17)$ & $C(22)$ & $126.0(7)$ & C(28) & $\mathrm{C}(23)$ & $C(24)$ & $118.2(4)$ \\
\hline C(23) & $C(24)$ & C(25) & $120.8(6)$ & C(23) & $C(28)$ & $C(27)$ & $120.3(5)$ \\
\hline C(24) & C(25) & $C(26)$ & $121.6(6)$ & C(25) & $C(26)$ & $C(27)$ & 118.9(6) \\
\hline C(26) & C(27) & C(28) & $120.1(7)$ & C(34) & C(29) & C(30) & $118.4(6)$ \\
\hline C(29) & C(30) & C(31) & $120.1(7)$ & C(29) & C(34) & C(33) & $121.8(6)$ \\
\hline C(30) & C(31) & C(32) & $120.6(7)$ & C(31) & C(32) & C(33) & 119.9(9) \\
\hline C(32) & C(33) & C(34) & 119.1(7) & $\mathrm{C}(41)$ & C(36) & C(37) & 119.2(6) \\
\hline$C(36)$ & C(37) & C(38) & $121.1(6)$ & C(36) & $C(41)$ & $C(40)$ & $119.5(6)$ \\
\hline C(37) & C(38) & C(39) & $119.9(6)$ & C(38) & C(39) & $C(40)$ & $119.7(8)$ \\
\hline C(39) & C(40) & $\mathrm{C}(41)$ & $120.5(7)$ & $\mathrm{C}(47)$ & $C(42)$ & $C(43)$ & $119.2(5)$ \\
\hline $\mathrm{C}(42)$ & C(43) & $C(44)$ & $119.6(7)$ & $\mathrm{C}(42)$ & $C(47)$ & $C(46)$ & $120.5(6)$ \\
\hline C(43) & $C(44)$ & $C(45)$ & $120.0(7)$ & $C(44)$ & $C(45)$ & $C(46)$ & $121.3(6)$ \\
\hline C(45) & $C(46)$ & $C(47)$ & 119.3(7) & C(48) & $\mathrm{C}(49)$ & $\mathrm{C}(50)$ & 118.8(9) \\
\hline$C(50)^{1)}$ & C(48) & C(49) & $119(1)$ & C(48) & $C(50)^{1)}$ & $\mathrm{C}(49)^{1)}$ & $121(1)$ \\
\hline C(49) & $C(50)$ & $C(48)^{1)}$ & 121(1) & $\mathrm{C}(50)$ & $C(48)^{1)}$ & $\mathrm{C}(49)^{1)}$ & 119(1) \\
\hline $\mathrm{C}(51)$ & $\mathrm{C}(52)$ & C(53) & $119.0(7)$ & $\mathrm{C}(53)^{2)}$ & $\mathrm{C}(51)$ & $\mathrm{C}(52)$ & $119.9(8)$ \\
\hline C(51) & $\mathrm{C}(53)^{2)}$ & $\mathrm{C}(52)^{2)}$ & $121.0(8)$ & $\mathrm{C}(52)$ & C(53) & $\mathrm{C}(51)^{2)}$ & $121.0(8)$ \\
\hline C(53) & $\mathrm{C}(51)^{2)}$ & $\mathrm{C}(52)^{2)}$ & $119.9(8)$ & C(54) & C(55) & C(56) & $119(2)$ \\
\hline$C(56)^{3)}$ & C(54) & C(55) & $117(1)$ & C(54) & $\mathrm{C}(56)^{3)}$ & $C(55)^{3)}$ & $122(2)$ \\
\hline C(55) & C(56) & $\mathrm{C}(54)^{3)}$ & $122(2)$ & C(56) & $C(54)^{3)}$ & $C(55)^{3)}$ & $117(1)$ \\
\hline
\end{tabular}

Angles are in degrees.

Symmetry operations: 1 ), $-\mathrm{X}+1,-\mathrm{Y}+1,-\mathrm{Z}$; 2), $-\mathrm{X},-\mathrm{Y},-\mathrm{Z}+1$; 3), $-\mathrm{X}+1,-\mathrm{Y}+2,-\mathrm{Z}$. 
Table S19. Positional Parameters and B(eq)

for $\left.\left[(C p * I r)_{2}\{\mathrm{Rh}(\operatorname{cod})\}\left(\mu_{3}-\mathrm{NCN}-N, N, N\right)\right\}_{2}\right]\left(\mathrm{BPh}_{4}\right)\left(\mathbf{1 0 a B P h}{ }_{4} \cdot \mathrm{CH}_{2} \mathrm{Cl}_{2}\right)$.

\begin{tabular}{|c|c|c|c|c|c|}
\hline atom & $\mathrm{x}$ & $\mathrm{y}$ & $\mathrm{Z}$ & $\mathrm{B}(\mathrm{eq})$ & occupancy \\
\hline $\operatorname{Ir}(1)$ & $0.20707(3)$ & $0.14770(3)$ & $0.14925(1)$ & $2.588(7)$ & \\
\hline $\mathrm{Rh}(1)$ & 0.2500 & 0.2500 & $0.22609(3)$ & $2.74(2)$ & $1 / 2$ \\
\hline $\mathrm{Cl}(1)$ & $0.6712(4)$ & $0.3303(5)$ & $0.2349(2)$ & $10.3(2)$ & \\
\hline $\mathrm{N}(1)$ & $0.3370(6)$ & $0.2123(6)$ & $0.1749(3)$ & $2.6(2)$ & \\
\hline $\mathrm{N}(2)$ & $0.5160(8)$ & $0.132(1)$ & $0.1764(4)$ & $6.0(3)$ & \\
\hline $\mathrm{C}(1)$ & $0.4328(8)$ & $0.1701(9)$ & $0.1752(4)$ & $3.5(2)$ & \\
\hline $\mathrm{C}(2)$ & $0.0696(9)$ & $0.0641(9)$ & $0.1274(4)$ & $3.9(3)$ & \\
\hline $\mathrm{C}(3)$ & $0.133(1)$ & $0.092(1)$ & $0.0929(4)$ & $4.4(3)$ & \\
\hline$C(4)$ & $0.2330(9)$ & $0.0436(9)$ & $0.0981(3)$ & $3.8(3)$ & \\
\hline $\mathrm{C}(5)$ & $0.232(1)$ & $-0.0135(9)$ & $0.1352(4)$ & $4.3(3)$ & \\
\hline $\mathrm{C}(6)$ & $0.132(1)$ & $-0.001(1)$ & $0.1534(5)$ & $5.5(4)$ & \\
\hline$C(7)$ & $-0.043(1)$ & 0.098(1) & $0.1347(5)$ & $6.8(4)$ & \\
\hline $\mathrm{C}(8)$ & 0.098(1) & $0.158(1)$ & $0.0577(5)$ & $6.4(4)$ & \\
\hline $\mathrm{C}(9)$ & $0.325(1)$ & 0.049(1) & $0.0686(5)$ & $6.5(4)$ & \\
\hline$C(10)$ & $0.319(1)$ & $-0.087(1)$ & $0.1518(6)$ & $8.9(6)$ & \\
\hline $\mathrm{C}(11)$ & $0.093(1)$ & $-0.053(1)$ & $0.1928(5)$ & $8.4(5)$ & \\
\hline $\mathrm{C}(12)$ & $0.249(2)$ & $0.118(2)$ & $0.3037(6)$ & $10.9(7)$ & \\
\hline C(13) & $0.329(1)$ & $0.155(1)$ & $0.2712(4)$ & $5.8(4)$ & \\
\hline$C(14)$ & $0.374(1)$ & $0.251(1)$ & $0.2713(4)$ & $5.5(3)$ & \\
\hline$C(15)$ & $0.356(2)$ & $0.338(2)$ & $0.3025(6)$ & $10.4(7)$ & \\
\hline$C(16)$ & $0.6452(7)$ & $0.2351(7)$ & $-0.0215(3)$ & $2.9(2)$ & \\
\hline $\mathrm{C}(17)$ & $0.5607(8)$ & $0.1686(8)$ & $-0.0120(3)$ & $3.4(2)$ & \\
\hline C(18) & $0.4711(8)$ & $0.1584(9)$ & $-0.0367(4)$ & $4.0(3)$ & \\
\hline C(19) & $0.4629(9)$ & $0.2140(9)$ & $-0.0729(4)$ & 4.3(3) & \\
\hline $\mathrm{C}(20)$ & $0.5430(9)$ & $0.2820(9)$ & $-0.0834(4)$ & $4.5(3)$ & \\
\hline $\mathrm{C}(21)$ & $0.6309(9)$ & $0.2919(8)$ & $-0.0586(4)$ & $3.5(2)$ & \\
\hline $\mathrm{C}(22)$ & $0.7621(8)$ & $0.1450(8)$ & $0.0364(3)$ & $3.1(2)$ & \\
\hline C(23) & $0.8270(9)$ & $0.0584(9)$ & $0.0259(4)$ & $3.8(3)$ & \\
\hline$C(24)$ & $0.829(1)$ & $-0.0335(9)$ & $0.0474(4)$ & $4.0(3)$ & \\
\hline C(25) & $0.766(1)$ & $-0.0463(9)$ & $0.0822(4)$ & $4.5(3)$ & \\
\hline $\mathrm{C}(26)$ & $0.703(1)$ & $0.036(1)$ & $0.0948(4)$ & $4.6(3)$ & \\
\hline $\mathrm{C}(27)$ & $0.7026(8)$ & $0.1269(9)$ & $0.0724(4)$ & $3.9(3)$ & \\
\hline C(28) & $0.75000(1)$ & $0.25000(1)$ & $0.2028(9)$ & $9.0(8)$ & $1 / 2$ \\
\hline $\mathrm{B}(1)$ & 0.7500 & 0.2500 & $0.0070(5)$ & $3.1(3)$ & $1 / 2$ \\
\hline $\mathrm{H}(1)$ & -0.0464 & 0.1723 & 0.1363 & 8.162 & \\
\hline $\mathrm{H}(2)$ & -0.0863 & 0.0741 & 0.1126 & 8.163 & \\
\hline $\mathrm{H}(3)$ & -0.0672 & 0.0684 & 0.1600 & 8.162 & \\
\hline $\mathrm{H}(4)$ & 0.0784 & 0.2253 & 0.0675 & 7.652 & \\
\hline $\mathrm{H}(5)$ & 0.1545 & 0.1645 & 0.0386 & 7.652 & \\
\hline $\mathrm{H}(6)$ & 0.0395 & 0.1258 & 0.0444 & 7.653 & \\
\hline $\mathrm{H}(7)$ & 0.3424 & -0.0193 & 0.0591 & 7.754 & \\
\hline $\mathrm{H}(8)$ & 0.3078 & 0.0925 & 0.0457 & 7.755 & \\
\hline $\mathrm{H}(9)$ & 0.3841 & 0.0783 & 0.0828 & 7.754 & \\
\hline $\mathrm{H}(10)$ & 0.2942 & -0.1573 & 0.1519 & 10.691 & \\
\hline $\mathrm{H}(11)$ & 0.3788 & -0.0814 & 0.1343 & 10.692 & \\
\hline $\mathrm{H}(12)$ & 0.3376 & -0.0666 & 0.1791 & 10.692 & \\
\hline $\mathrm{H}(13)$ & 0.0709 & -0.0005 & 0.2120 & 10.136 & \\
\hline $\mathrm{H}(14)$ & 0.0357 & -0.0976 & 0.1865 & 10.137 & \\
\hline $\mathrm{H}(15)$ & 0.1488 & -0.0927 & 0.2047 & 10.136 & \\
\hline $\mathrm{H}(16)$ & 0.2416 & 0.0445 & 0.3005 & 13.068 & \\
\hline $\mathrm{H}(17)$ & 0.2796 & 0.1330 & 0.3300 & 13.069 & \\
\hline $\mathrm{H}(18)$ & 0.3461 & 0.1082 & 0.2494 & 6.996 & \\
\hline $\mathrm{H}(19)$ & 0.4218 & 0.2670 & 0.2495 & 6.588 & \\
\hline $\mathrm{H}(20)$ & 0.3693 & 0.3084 & 0.3290 & 12.435 & \\
\hline
\end{tabular}




\begin{tabular}{lrrrr}
$\mathrm{H}(21)$ & 0.4056 & 0.3931 & 0.2975 & 12.435 \\
$\mathrm{H}(22)$ & 0.5645 & 0.1280 & 0.0126 & 4.056 \\
$\mathrm{H}(23)$ & 0.4156 & 0.1129 & -0.0284 & 4.832 \\
$\mathrm{H}(24)$ & 0.4035 & 0.2054 & -0.0905 & 5.113 \\
$\mathrm{H}(25)$ & 0.5374 & 0.3229 & -0.1079 & 5.345 \\
$\mathrm{H}(26)$ & 0.6847 & 0.3393 & -0.0670 & 4.206 \\
$\mathrm{H}(27)$ & 0.8713 & 0.0647 & 0.0023 & 4.615 \\
$\mathrm{H}(28)$ & 0.8742 & -0.0889 & 0.0386 & 4.855 \\
$\mathrm{H}(29)$ & 0.7664 & -0.1105 & 0.0972 & 5.411 \\
$\mathrm{H}(30)$ & 0.6603 & 0.0291 & 0.1187 & 5.509 \\
$\mathrm{H}(31)$ & 0.6589 & 0.1823 & 0.0821 & 4.646 \\
$\mathrm{H}(32)$ & 0.7062 & 0.2075 & 0.1858 & 10.766 \\
\hline
\end{tabular}

Table S20. Anisotropic Displacement Parameters for $\left[(\mathrm{Cp} * \mathrm{Ir})_{2}\{\mathrm{Rh}(\mathrm{cod})\}\left(\mu_{3}-\mathrm{NCN}-N, N, N\right)_{2}\right]\left(\mathrm{BPh}_{4}\right)\left(\mathbf{1 0 a B P h}{ }_{4} \cdot \mathrm{CH}_{2} \mathrm{Cl}_{2}\right)$.

\begin{tabular}{|c|c|c|c|c|c|c|}
\hline atom & U11 & U22 & U33 & U12 & U13 & U23 \\
\hline $\operatorname{Ir}(1)$ & $0.0331(2)$ & $0.0370(2)$ & $0.0283(2)$ & $-0.0047(2)$ & $-0.0005(2)$ & $-0.0030(2)$ \\
\hline $\operatorname{Rh}(1)$ & $0.0399(5)$ & $0.0428(6)$ & $0.0213(5)$ & $0.0037(5)$ & 0.0000 & 0.0000 \\
\hline $\mathrm{Cl}(1)$ & $0.127(4)$ & $0.160(5)$ & $0.105(4)$ & $0.014(4)$ & $-0.020(3)$ & $-0.036(4)$ \\
\hline $\mathrm{N}(1)$ & $0.030(4)$ & $0.036(4)$ & $0.032(5)$ & $-0.003(3)$ & $0.005(4)$ & $-0.007(4)$ \\
\hline $\mathrm{N}(2)$ & $0.047(6)$ & $0.092(9)$ & $0.091(9)$ & $0.014(6)$ & $-0.005(6)$ & $-0.017(7)$ \\
\hline $\mathrm{C}(1)$ & $0.036(5)$ & $0.060(7)$ & $0.038(6)$ & $0.001(5)$ & $-0.007(5)$ & $-0.005(5)$ \\
\hline $\mathrm{C}(2)$ & $0.054(7)$ & $0.055(7)$ & $0.038(7)$ & $-0.010(6)$ & $-0.005(5)$ & $-0.011(6)$ \\
\hline C(3) & $0.068(8)$ & $0.055(7)$ & $0.043(7)$ & $-0.004(6)$ & $-0.014(6)$ & $-0.009(6)$ \\
\hline C(4) & $0.054(8)$ & $0.052(6)$ & $0.039(6)$ & $-0.009(6)$ & $0.009(5)$ & $-0.010(5)$ \\
\hline C(5) & $0.061(8)$ & $0.039(6)$ & $0.064(8)$ & $-0.013(6)$ & $-0.025(6)$ & $0.002(6)$ \\
\hline C(6) & $0.09(1)$ & $0.056(7)$ & $0.063(9)$ & $-0.026(7)$ & $0.001(8)$ & $-0.004(7)$ \\
\hline C(7) & $0.050(8)$ & $0.13(1)$ & $0.08(1)$ & $-0.013(9)$ & $-0.009(7)$ & $-0.02(1)$ \\
\hline C(8) & $0.08(1)$ & $0.09(1)$ & $0.07(1)$ & $-0.013(9)$ & $-0.019(8)$ & $-0.018(9)$ \\
\hline C(9) & 0.09(1) & $0.072(9)$ & 0.09(1) & $-0.005(8)$ & $0.019(9)$ & $-0.032(9)$ \\
\hline $\mathrm{C}(10)$ & $0.13(1)$ & $0.059(9)$ & $0.15(2)$ & $0.03(1)$ & $-0.04(1)$ & $-0.02(1)$ \\
\hline $\mathrm{C}(11)$ & $0.15(2)$ & $0.11(1)$ & $0.06(1)$ & $-0.06(1)$ & $-0.00(1)$ & $0.02(1)$ \\
\hline $\mathrm{C}(12)$ & $0.17(2)$ & $0.14(2)$ & $0.10(2)$ & $0.02(2)$ & $0.02(2)$ & $0.08(1)$ \\
\hline$C(13)$ & $0.09(1)$ & $0.08(1)$ & 0.048(8) & $0.015(9)$ & $-0.004(7)$ & $0.017(8)$ \\
\hline $\mathrm{C}(14)$ & $0.073(8)$ & $0.10(1)$ & $0.035(7)$ & $0.011(9)$ & $-0.022(7)$ & $-0.004(8)$ \\
\hline $\mathrm{C}(15)$ & $0.12(2)$ & $0.15(2)$ & $0.12(2)$ & $0.04(2)$ & $-0.06(1)$ & $-0.08(2)$ \\
\hline $\mathrm{C}(16)$ & $0.035(5)$ & $0.032(5)$ & $0.042(6)$ & $0.001(4)$ & $0.000(5)$ & $-0.003(5)$ \\
\hline $\mathrm{C}(17)$ & $0.043(6)$ & $0.045(6)$ & $0.041(6)$ & $-0.011(5)$ & $0.002(5)$ & $0.005(5)$ \\
\hline $\mathrm{C}(18)$ & $0.037(6)$ & $0.049(6)$ & $0.067(8)$ & $-0.013(5)$ & $-0.002(5)$ & $0.000(6)$ \\
\hline C(19) & $0.041(6)$ & $0.062(8)$ & $0.059(8)$ & $-0.002(5)$ & $-0.013(6)$ & $-0.005(6)$ \\
\hline C(20) & $0.055(7)$ & $0.057(8)$ & $0.058(8)$ & $-0.002(6)$ & $-0.012(6)$ & $0.014(6)$ \\
\hline $\mathrm{C}(21)$ & $0.046(6)$ & $0.037(6)$ & $0.050(7)$ & $-0.009(5)$ & $-0.006(5)$ & $0.004(5)$ \\
\hline$C(22)$ & $0.038(5)$ & $0.049(6)$ & $0.032(5)$ & $-0.011(5)$ & $-0.004(4)$ & $0.003(5)$ \\
\hline C(23) & $0.051(6)$ & $0.051(6)$ & $0.044(7)$ & $-0.001(6)$ & $-0.008(5)$ & $-0.001(6)$ \\
\hline $\mathrm{C}(24)$ & $0.067(7)$ & $0.051(7)$ & $0.036(6)$ & $0.009(6)$ & $-0.012(6)$ & $-0.004(5)$ \\
\hline C(25) & $0.069(8)$ & $0.043(6)$ & $0.060(8)$ & $-0.015(6)$ & $-0.025(7)$ & $0.007(6)$ \\
\hline C(26) & $0.053(7)$ & $0.076(9)$ & $0.045(7)$ & $-0.019(7)$ & $-0.004(6)$ & $0.018(6)$ \\
\hline $\mathrm{C}(27)$ & $0.042(6)$ & $0.056(7)$ & $0.049(7)$ & $0.001(6)$ & $-0.002(6)$ & $0.006(5)$ \\
\hline C(28) & $0.08(1)$ & $0.14(2)$ & $0.12(2)$ & $-0.03(2)$ & 0.0000 & 0.0000 \\
\hline $\mathrm{B}(1)$ & $0.045(9)$ & $0.038(8)$ & $0.036(9)$ & $-0.007(8)$ & 0.0000 & 0.0000 \\
\hline
\end{tabular}


Table S21. Intramolecular Distances Involving the Nonhydrogen Atoms for $\left[(\mathrm{Cp} * \mathrm{Ir})_{2}\{\mathrm{Rh}(\mathrm{cod})\}\left(\mu_{3}-\mathrm{NCN}-N, N, N\right)_{2}\right]\left(\mathrm{BPh}_{4}\right)\left(\mathbf{1 0 a B P h}{ }_{4} \cdot \mathrm{CH}_{2} \mathrm{Cl}_{2}\right)$.

\begin{tabular}{|c|c|c|c|c|c|}
\hline atom & atom & distance & atom & atom & distance \\
\hline $\operatorname{Ir}(1)$ & $\operatorname{Ir}(1)^{1)}$ & $2.8237(5)$ & $\operatorname{Ir}(1)$ & $\mathrm{Rh}(1)$ & $2.8552(9)$ \\
\hline $\operatorname{Ir}(1)$ & $\mathrm{N}(1)$ & $2.019(8)$ & $\operatorname{Ir}(1)$ & $\mathrm{N}(1)^{1)}$ & $2.044(8)$ \\
\hline $\operatorname{Ir}(1)$ & $\mathrm{C}(2)$ & $2.16(1)$ & $\operatorname{Ir}(1)$ & $\mathrm{C}(3)$ & $2.17(1)$ \\
\hline $\operatorname{Ir}(1)$ & $C(4)$ & $2.14(1)$ & $\operatorname{Ir}(1)$ & C(5) & $2.12(1)$ \\
\hline $\operatorname{Ir}(1)$ & C(6) & $2.12(1)$ & $\mathrm{Rh}(1)$ & $\operatorname{Ir}(1)^{1)}$ & $2.8552(9)$ \\
\hline $\operatorname{Rh}(1)$ & $\mathrm{N}(1)$ & $2.046(8)$ & Rh(1) & $\mathrm{N}(1)^{1)}$ & $2.046(8)$ \\
\hline $\operatorname{Rh}(1)$ & C(13) & $2.14(1)$ & Rh(1) & $C(13)^{1)}$ & $2.14(1)$ \\
\hline $\operatorname{Rh}(1)$ & $C(14)$ & $2.15(1)$ & Rh(1) & $C(14)^{1)}$ & $2.15(1)$ \\
\hline $\mathrm{Cl}(1)$ & C(28) & $1.77(2)$ & $\mathrm{N}(1)$ & $\left.\operatorname{Ir}(1)^{1}\right)$ & $2.044(8)$ \\
\hline $\mathrm{N}(1)$ & $\mathrm{C}(1)$ & 1.33(1) & $\mathrm{N}(2)$ & $\mathrm{C}(1)$ & $1.16(1)$ \\
\hline $\mathrm{C}(2)$ & C(3) & $1.42(2)$ & $C(2)$ & C(6) & $1.42(2)$ \\
\hline $\mathrm{C}(2)$ & $\mathrm{C}(7)$ & $1.51(2)$ & $\mathrm{C}(3)$ & $C(4)$ & $1.43(2)$ \\
\hline C(3) & $\mathrm{C}(8)$ & $1.48(2)$ & $C(4)$ & C(5) & $1.40(2)$ \\
\hline C(4) & C(9) & $1.51(2)$ & $\mathrm{C}(5)$ & $C(6)$ & $1.41(2)$ \\
\hline C(5) & C(10) & $1.54(2)$ & C(6) & $\mathrm{C}(11)$ & $1.52(2)$ \\
\hline C(10) & C(5) & $1.54(2)$ & $\mathrm{C}(12)$ & C(13) & $1.53(3)$ \\
\hline $\mathrm{C}(12)$ & $C(15)^{1)}$ & $1.45(3)$ & $C(13)$ & $\mathrm{C}(14)$ & $1.36(2)$ \\
\hline$C(14)$ & C(15) & $1.51(3)$ & $\mathrm{C}(15)$ & $C(12)^{1)}$ & $1.45(3)$ \\
\hline $\mathrm{C}(16)$ & $\mathrm{C}(17)$ & $1.40(1)$ & $\mathrm{C}(16)$ & $\mathrm{C}(21)$ & $1.41(2)$ \\
\hline $\mathrm{C}(16)$ & $\mathrm{B}(1)$ & 1.63(1) & $\mathrm{C}(17)$ & C(18) & $1.40(2)$ \\
\hline C(18) & C(19) & $1.37(2)$ & C(19) & C(20) & $1.38(2)$ \\
\hline C(20) & $\mathrm{C}(21)$ & $1.38(2)$ & $\mathrm{C}(22)$ & C(23) & 1.42(2) \\
\hline $\mathrm{C}(22)$ & $\mathrm{C}(27)$ & $1.40(2)$ & $\mathrm{C}(22)$ & $\mathrm{B}(1)$ & $1.65(1)$ \\
\hline C(23) & C(24) & $1.36(2)$ & $\mathrm{C}(24)$ & C(25) & 1.39(2) \\
\hline$C(25)$ & $C(26)$ & $1.38(2)$ & $C(26)$ & C(27) & $1.37(2)$ \\
\hline C(28) & $\mathrm{Cl}(1)^{2)}$ & $1.77(2)$ & $\mathrm{B}(1)$ & $C(16)^{2)}$ & $1.63(1)$ \\
\hline $\mathrm{B}(1)$ & $C(22)^{2)}$ & $1.65(1)$ & & & \\
\hline
\end{tabular}

Distances are in angstroms.

Symmetry operations: 1 ), $-\mathrm{X}+1 / 2,-\mathrm{Y}+1 / 2, \mathrm{Z} ; 2),-\mathrm{X}+1 / 2+1,-\mathrm{Y}+1 / 2, \mathrm{Z}$. 
Table S22. Intramolecular Bond Angles Involving the Nonhydrogen Atoms for $\left[(\mathrm{Cp} * \mathrm{Ir})_{2}\{\mathrm{Rh}(\mathrm{cod})\}\left(\mu_{3}-\mathrm{NCN}-N, N, N\right)_{2}\right]\left(\mathrm{BPh}_{4}\right)\left(\mathbf{1 0 a B P h}{ }_{4} \cdot \mathrm{CH}_{2} \mathrm{Cl}_{2}\right)$.

\begin{tabular}{|c|c|c|c|c|c|c|c|}
\hline atom & atom & atom & angle & atom & atom & atom & angle \\
\hline $\operatorname{Ir}(1)$ & $\operatorname{Ir}(1)^{1)}$ & $\mathrm{Rh}(1)$ & $60.36(1)$ & $\operatorname{Rh}(1)$ & $\operatorname{Ir}(1)$ & $\operatorname{Ir}(1)^{1)}$ & $60.36(1)$ \\
\hline $\operatorname{Ir}(1)$ & $\operatorname{Ir}(1)^{1)}$ & $\mathrm{N}(1)$ & $45.6(2)$ & $\mathrm{N}(1)$ & $\operatorname{Ir}(1)$ & $\operatorname{Ir}(1)^{1)}$ & $46.3(2)$ \\
\hline $\operatorname{Ir}(1)$ & $\operatorname{Ir}(1)^{1)}$ & $\mathrm{N}(1)^{1)}$ & $46.3(2)$ & $\mathrm{N}(1)^{1)}$ & $\operatorname{Ir}(1)$ & $\operatorname{Ir}(1)^{1)}$ & $45.6(2)$ \\
\hline $\mathrm{C}(2)$ & $\operatorname{Ir}(1)$ & $\operatorname{Ir}(1)^{1)}$ & $140.0(3)$ & C(3) & $\operatorname{Ir}(1)$ & $\operatorname{Ir}(1)^{1)}$ & $117.9(3)$ \\
\hline $\mathrm{C}(4)$ & $\operatorname{Ir}(1)$ & $\operatorname{Ir}(1)^{1)}$ & $120.8(3)$ & $\mathrm{C}(5)$ & $\operatorname{Ir}(1)$ & $\operatorname{Ir}(1)^{1)}$ & $146.6(3)$ \\
\hline C(6) & $\operatorname{Ir}(1)$ & $\operatorname{Ir}(1)^{1)}$ & $174.5(4)$ & $\operatorname{Ir}(1)$ & $\operatorname{Ir}(1)^{1)}$ & $C(2)^{1)}$ & $140.0(3)$ \\
\hline $\operatorname{Ir}(1)$ & $\mathrm{Rh}(1)$ & $\operatorname{Ir}(1)^{1)}$ & $59.27(2)$ & $\operatorname{Ir}(1)$ & $\operatorname{Rh}(1)$ & $\mathrm{N}(1)$ & $45.0(2)$ \\
\hline $\mathrm{N}(1)$ & $\operatorname{Ir}(1)$ & $\mathrm{Rh}(1)$ & $45.8(2)$ & $\operatorname{Ir}(1)$ & Rh(1) & $\mathrm{N}(1)^{1)}$ & $45.7(2)$ \\
\hline $\mathrm{N}(1)^{1)}$ & $\operatorname{Ir}(1)$ & $\operatorname{Rh}(1)$ & $45.8(2)$ & $\mathrm{C}(2)$ & $\operatorname{Ir}(1)$ & Rh(1) & 131.5(3) \\
\hline C(3) & $\operatorname{Ir}(1)$ & $\mathrm{Rh}(1)$ & $164.0(3)$ & $\mathrm{C}(4)$ & $\operatorname{Ir}(1)$ & $\mathrm{Rh}(1)$ & 157.3(3) \\
\hline $\mathrm{C}(5)$ & $\operatorname{Ir}(1)$ & $\operatorname{Rh}(1)$ & $126.8(4)$ & $\mathrm{C}(6)$ & $\operatorname{Ir}(1)$ & $\operatorname{Rh}(1)$ & $115.9(4)$ \\
\hline $\operatorname{Ir}(1)$ & $\operatorname{Rh}(1)$ & $\mathrm{C}(13)$ & $115.0(4)$ & $\operatorname{Ir}(1)$ & $\operatorname{Rh}(1)$ & $C(13)^{1)}$ & $139.6(4)$ \\
\hline $\operatorname{Ir}(1)$ & $\operatorname{Rh}(1)$ & $C(14)$ & $137.3(4)$ & $\operatorname{Ir}(1)$ & $\mathrm{Rh}(1)$ & $\mathrm{C}(14)^{1)}$ & $116.6(4)$ \\
\hline $\operatorname{Ir}(1)$ & $\mathrm{N}(1)$ & $\operatorname{Ir}(1)^{1)}$ & 88.1(3) & $\operatorname{Ir}(1)$ & $\mathrm{N}(1)$ & $\mathrm{Rh}(1)$ & 89.2(3) \\
\hline $\mathrm{N}(1)^{1)}$ & $\operatorname{Ir}(1)$ & $\mathrm{N}(1)$ & $72.7(3)$ & $\operatorname{Ir}(1)$ & $\mathrm{N}(1)$ & $\mathrm{C}(1)$ & $125.8(7)$ \\
\hline$C(2)$ & $\operatorname{Ir}(1)$ & $\mathrm{N}(1)$ & $173.2(4)$ & $\mathrm{C}(3)$ & $\operatorname{Ir}(1)$ & $\mathrm{N}(1)$ & $146.3(4)$ \\
\hline C(4) & $\operatorname{Ir}(1)$ & $\mathrm{N}(1)$ & $116.2(4)$ & $\mathrm{C}(5)$ & $\operatorname{Ir}(1)$ & $\mathrm{N}(1)$ & $111.2(4)$ \\
\hline$C(6)$ & $\operatorname{Ir}(1)$ & $N(1)$ & $134.8(5)$ & $\operatorname{Ir}(1)$ & $\mathrm{N}(1)^{1)}$ & $\operatorname{Ir}(1)^{1)}$ & 88.1(3) \\
\hline $\operatorname{Ir}(1)$ & $\mathrm{N}(1)^{1)}$ & $\operatorname{Rh}(1)$ & 88.6(3) & $\operatorname{Ir}(1)$ & $\mathrm{N}(1)^{1)}$ & $C(1)^{1)}$ & 127.3(7) \\
\hline $\mathrm{C}(2)$ & $\operatorname{Ir}(1)$ & $\mathrm{N}(1)^{1)}$ & $110.0(4)$ & $\mathrm{C}(3)$ & $\operatorname{Ir}(1)$ & $\mathrm{N}(1)^{1)}$ & $120.3(4)$ \\
\hline $\mathrm{C}(4)$ & $\operatorname{Ir}(1)$ & $\mathrm{N}(1)^{1)}$ & $153.3(4)$ & $\mathrm{C}(5)$ & $\operatorname{Ir}(1)$ & $\mathrm{N}(1)^{1)}$ & $165.8(4)$ \\
\hline$C(6)$ & $\operatorname{Ir}(1)$ & $\mathrm{N}(1)^{1)}$ & $128.9(5)$ & $\operatorname{Ir}(1)$ & $\mathrm{C}(2)$ & $\mathrm{C}(3)$ & 71.2(7) \\
\hline C(3) & $\operatorname{Ir}(1)$ & $\mathrm{C}(2)$ & $38.2(5)$ & $C(4)$ & $\operatorname{Ir}(1)$ & $C(2)$ & $64.4(4)$ \\
\hline $\mathrm{C}(5)$ & $\operatorname{Ir}(1)$ & $\mathrm{C}(2)$ & $64.7(5)$ & $\operatorname{Ir}(1)$ & $\mathrm{C}(2)$ & C(6) & 69.2(7) \\
\hline C(6) & $\operatorname{Ir}(1)$ & $C(2)$ & $38.6(5)$ & $\operatorname{Ir}(1)$ & $\mathrm{C}(2)$ & $\mathrm{C}(7)$ & 124.8(9) \\
\hline $\operatorname{Ir}(1)$ & $\mathrm{C}(3)$ & $C(2)$ & 70.6(7) & $\operatorname{Ir}(1)$ & $\mathrm{C}(3)$ & $C(4)$ & 69.8(7) \\
\hline C(4) & $\operatorname{Ir}(1)$ & $\mathrm{C}(3)$ & $38.6(5)$ & $C(5)$ & $\operatorname{Ir}(1)$ & $\mathrm{C}(3)$ & $64.5(5)$ \\
\hline C(6) & $\operatorname{Ir}(1)$ & $\mathrm{C}(3)$ & $64.3(5)$ & $\operatorname{Ir}(1)$ & $\mathrm{C}(3)$ & $\mathrm{C}(8)$ & 126.2(9) \\
\hline $\operatorname{Ir}(1)$ & $\mathrm{C}(4)$ & $\mathrm{C}(3)$ & 71.6(7) & $\operatorname{Ir}(1)$ & $C(4)$ & $\mathrm{C}(5)$ & 70.1(7) \\
\hline C(5) & $\operatorname{Ir}(1)$ & $C(4)$ & $38.3(5)$ & C(6) & $\operatorname{Ir}(1)$ & $C(4)$ & $64.4(5)$ \\
\hline $\operatorname{Ir}(1)$ & $C(4)$ & C(9) & 125.1(9) & $\operatorname{Ir}(1)$ & $\mathrm{C}(5)$ & $\mathrm{C}(4)$ & $71.6(6)$ \\
\hline $\operatorname{Ir}(1)$ & C(5) & $\mathrm{C}(6)$ & 70.5(7) & C $(6)$ & $\operatorname{Ir}(1)$ & $\mathrm{C}(5)$ & $38.7(5)$ \\
\hline $\operatorname{Ir}(1)$ & $\mathrm{C}(5)$ & $C(10)$ & 127.9(9) & $\operatorname{Ir}(1)$ & $\mathrm{C}(6)$ & $\mathrm{C}(2)$ & $72.2(7)$ \\
\hline $\operatorname{Ir}(1)$ & C(6) & C(5) & 70.8(7) & $\operatorname{Ir}(1)$ & C(6) & $\mathrm{C}(11)$ & $125(1)$ \\
\hline $\operatorname{Rh}(1)$ & $\operatorname{Ir}(1)^{1)}$ & $\mathrm{N}(1)$ & $45.8(2)$ & $\mathrm{N}(1)$ & $\mathrm{Rh}(1)$ & $\operatorname{Ir}(1)^{1)}$ & $45.7(2)$ \\
\hline $\operatorname{Rh}(1)$ & $\operatorname{Ir}(1)^{1)}$ & $\mathrm{N}(1)^{1)}$ & $45.8(2)$ & $\mathrm{N}(1)^{1)}$ & $\mathrm{Rh}(1)$ & $\operatorname{Ir}(1)^{1)}$ & $45.0(2)$ \\
\hline $\mathrm{C}(13)$ & $\mathrm{Rh}(1)$ & $\operatorname{Ir}(1)^{1)}$ & $139.6(4)$ & $\mathrm{C}(13)^{1)}$ & Rh(1) & $\operatorname{Ir}(1)^{1)}$ & $115.0(4)$ \\
\hline$C(14)$ & $\operatorname{Rh}(1)$ & $\operatorname{Ir}(1)^{1)}$ & $116.6(4)$ & $C(14)^{1)}$ & $\operatorname{Rh}(1)$ & $\operatorname{Ir}(1)^{1)}$ & $137.3(4)$ \\
\hline $\operatorname{Rh}(1)$ & $\operatorname{Ir}(1)^{1)}$ & $\mathrm{C}(2)^{1)}$ & $131.5(3)$ & $\mathrm{Rh}(1)$ & $\mathrm{N}(1)$ & $\operatorname{Ir}(1)^{1)}$ & 88.6(3) \\
\hline $\mathrm{N}(1)^{1)}$ & $\mathrm{Rh}(1)$ & $\mathrm{N}(1)$ & 72.1(3) & $\operatorname{Rh}(1)$ & $\mathrm{N}(1)$ & $\mathrm{C}(1)$ & $125.6(7)$ \\
\hline C(13) & $\mathrm{Rh}(1)$ & $\mathrm{N}(1)$ & $99.6(4)$ & $C(13)^{1)}$ & $\mathrm{Rh}(1)$ & $\mathrm{N}(1)$ & $159.2(5)$ \\
\hline$C(14)$ & Rh(1) & $\mathrm{N}(1)$ & $99.0(4)$ & $C(14)^{1)}$ & $\mathrm{Rh}(1)$ & $\mathrm{N}(1)$ & $160.7(5)$ \\
\hline Rh(1) & $\mathrm{N}(1)^{1)}$ & $\operatorname{Ir}(1)^{1)}$ & $89.2(3)$ & $\mathrm{Rh}(1)$ & $\mathrm{N}(1)^{1)}$ & $\mathrm{C}(1)^{1)}$ & $125.6(7)$ \\
\hline $\mathrm{C}(13)$ & $\mathrm{Rh}(1)$ & $\mathrm{N}(1)^{1)}$ & $159.2(5)$ & $C(13)^{1)}$ & $\mathrm{Rh}(1)$ & $\mathrm{N}(1)^{1)}$ & $99.6(4)$ \\
\hline$C(14)$ & Rh(1) & $\mathrm{N}(1)^{1)}$ & $160.7(5)$ & $\mathrm{C}(14)^{1)}$ & $\mathrm{Rh}(1)$ & $\mathrm{N}(1)^{1)}$ & $99.0(4)$ \\
\hline $\mathrm{Rh}(1)$ & $\mathrm{C}(13)$ & $\mathrm{C}(12)$ & $109(1)$ & $C(13)^{1)}$ & $\mathrm{Rh}(1)$ & C(13) & $94.1(6)$ \\
\hline $\mathrm{Rh}(1)$ & $\mathrm{C}(13)$ & $C(14)$ & $71.9(9)$ & C(14) & $\mathrm{Rh}(1)$ & C(13) & $36.8(6)$ \\
\hline$C(14)^{1)}$ & $\mathrm{Rh}(1)$ & $\mathrm{C}(13)$ & $82.7(5)$ & $\operatorname{Rh}(1)$ & $C(13)^{1)}$ & $C(12)^{1)}$ & $109(1)$ \\
\hline C(14) & $\operatorname{Rh}(1)$ & $C(13)^{1)}$ & $82.7(5)$ & $\operatorname{Rh}(1)$ & $C(13)^{1)}$ & $C(14)^{1)}$ & $71.9(9)$ \\
\hline$C(14)^{1)}$ & $\operatorname{Rh}(1)$ & $C(13)^{1)}$ & $36.8(6)$ & $\operatorname{Rh}(1)$ & $\mathrm{C}(14)$ & $\mathrm{C}(13)$ & 71.2(8) \\
\hline$C(14)^{1)}$ & $\mathrm{Rh}(1)$ & $\mathrm{C}(14)$ & $94.3(5)$ & $\operatorname{Rh}(1)$ & $\mathrm{C}(14)$ & $\mathrm{C}(15)$ & $110(1)$ \\
\hline $\mathrm{Rh}(1)$ & $C(14)^{1)}$ & $C(13)^{1)}$ & 71.2(8) & $\operatorname{Rh}(1)$ & $C(14)^{1)}$ & $C(15)^{1)}$ & $110(1)$ \\
\hline $\mathrm{Cl}(1)$ & $\mathrm{C}(28)$ & $\mathrm{Cl}(1)^{1)}$ & $108(1)$ & $\mathrm{N}(1)$ & $\operatorname{Ir}(1)^{1)}$ & $\mathrm{N}(1)^{1)}$ & $72.7(3)$ \\
\hline $\mathrm{C}(1)$ & $\mathrm{N}(1)$ & $\operatorname{Ir}(1)^{1)}$ & $127.3(7)$ & $\mathrm{N}(1)$ & $\operatorname{Ir}(1)^{1)}$ & $C(2)^{1)}$ & $110.0(4)$ \\
\hline
\end{tabular}




\begin{tabular}{|c|c|c|c|c|c|c|c|}
\hline $\mathrm{N}(1)$ & $\mathrm{C}(1)$ & $\mathrm{N}(2)$ & $178(1)$ & $C(2)$ & $\mathrm{C}(3)$ & $C(4)$ & $107(1)$ \\
\hline C(6) & $C(2)$ & $\mathrm{C}(3)$ & 107(1) & $C(7)$ & $C(2)$ & $\mathrm{C}(3)$ & $125(1)$ \\
\hline $\mathrm{C}(2)$ & $\mathrm{C}(3)$ & $\mathrm{C}(8)$ & $125(1)$ & $C(2)$ & C(6) & $\mathrm{C}(5)$ & $108(1)$ \\
\hline $\mathrm{C}(7)$ & $C(2)$ & $\mathrm{C}(6)$ & $126(1)$ & $C(2)$ & $C(6)$ & $\mathrm{C}(11)$ & $125(1)$ \\
\hline $\mathrm{C}(3)$ & C(4) & $C(5)$ & $108(1)$ & $\mathrm{C}(8)$ & $\mathrm{C}(3)$ & $C(4)$ & $126(1)$ \\
\hline C(3) & C(4) & $\mathrm{C}(9)$ & $126(1)$ & $C(4)$ & $C(5)$ & $\mathrm{C}(6)$ & $108(1)$ \\
\hline C(9) & $C(4)$ & $\mathrm{C}(5)$ & 124(1) & $C(4)$ & $C(5)$ & $C(10)$ & $126(1)$ \\
\hline C(10) & $\mathrm{C}(5)$ & C(6) & 124(1) & C(5) & $\mathrm{C}(6)$ & $C(11)$ & $126(1)$ \\
\hline $\mathrm{C}(12)$ & C(13) & C(14) & 123(1) & $C(15)^{1)}$ & $C(12)$ & $C(13)$ & 118(1) \\
\hline$C(12)$ & $C(15)^{1)}$ & $C(14)^{1)}$ & 115(1) & $\mathrm{C}(13)$ & $\mathrm{C}(14)$ & $\mathrm{C}(15)$ & $126(1)$ \\
\hline$C(14)$ & C(15) & $C(12)^{1)}$ & $115(1)$ & $C(15)$ & $C(12)^{1)}$ & $\mathrm{C}(13)^{1)}$ & $118(1)$ \\
\hline $\mathrm{C}(16)$ & $\mathrm{C}(17)$ & C(18) & $123(1)$ & $\mathrm{C}(21)$ & $\mathrm{C}(16)$ & $\mathrm{C}(17)$ & $113.5(9)$ \\
\hline $\mathrm{B}(1)$ & $C(16)$ & $C(17)$ & $124.8(9)$ & $C(16)$ & $\mathrm{C}(21)$ & C(20) & $123(1)$ \\
\hline $\mathrm{B}(1)$ & C(16) & $\mathrm{C}(21)$ & 121.7(9) & $\mathrm{C}(16)$ & $\mathrm{B}(1)$ & $C(16)^{1)}$ & $110(1)$ \\
\hline$C(16)$ & $\mathrm{B}(1)$ & $\mathrm{C}(22)$ & 107.9(5) & $\mathrm{C}(16)$ & $\mathrm{B}(1)$ & $C(22)^{1)}$ & $110.2(5)$ \\
\hline $\mathrm{C}(17)$ & $\mathrm{C}(18)$ & $\mathrm{C}(19)$ & $120(1)$ & $\mathrm{C}(18)$ & $\mathrm{C}(19)$ & C(20) & $118(1)$ \\
\hline C(19) & $\mathrm{C}(20)$ & $\mathrm{C}(21)$ & $120(1)$ & $\mathrm{C}(27)$ & $\mathrm{C}(22)$ & $C(23)$ & 112(1) \\
\hline $\mathrm{B}(1)$ & C(22) & C(23) & $123.3(9)$ & $C(22)$ & C(23) & $C(24)$ & 124(1) \\
\hline $\mathrm{B}(1)$ & $\mathrm{C}(22)$ & $\mathrm{C}(27)$ & 124.1(9) & $\mathrm{C}(22)$ & $\mathrm{C}(27)$ & $\mathrm{C}(26)$ & 125(1) \\
\hline $\mathrm{C}(22)$ & $\mathrm{B}(1)$ & $C(16)^{1)}$ & $110.2(5)$ & $\mathrm{C}(22)$ & $\mathrm{B}(1)$ & $\mathrm{C}(22)^{1)}$ & 109(1) \\
\hline C(23) & $C(24)$ & C(25) & $120(1)$ & $\mathrm{C}(24)$ & C(25) & C(26) & 118(1) \\
\hline C(25) & $\mathrm{C}(26)$ & C(27) & 119(1) & $\mathrm{B}(1)$ & $C(16)^{2)}$ & $\mathrm{C}(21)^{2)}$ & $121.7(9)$ \\
\hline$C(22)^{2)}$ & $\mathrm{B}(1)$ & $\mathrm{C}(16)^{2)}$ & $107.9(5)$ & $\mathrm{B}(1)$ & $C(16)^{2)}$ & $\mathrm{C}(17)^{2)}$ & 124.8(9) \\
\hline $\mathrm{B}(1)$ & $\mathrm{C}(22)^{2)}$ & $\mathrm{C}(23)^{2)}$ & 123.3(9) & $\mathrm{B}(1)$ & $\mathrm{C}(22)^{2)}$ & $\mathrm{C}(27)^{2)}$ & 124.1(9) \\
\hline
\end{tabular}

Angles are in degrees.

Symmetry operations: 1 ), $-\mathrm{X}+1 / 2,-\mathrm{Y}+1 / 2, \mathrm{Z}$; 2), $-\mathrm{X}+1 / 2+1,-\mathrm{Y}+1 / 2$, Z. 


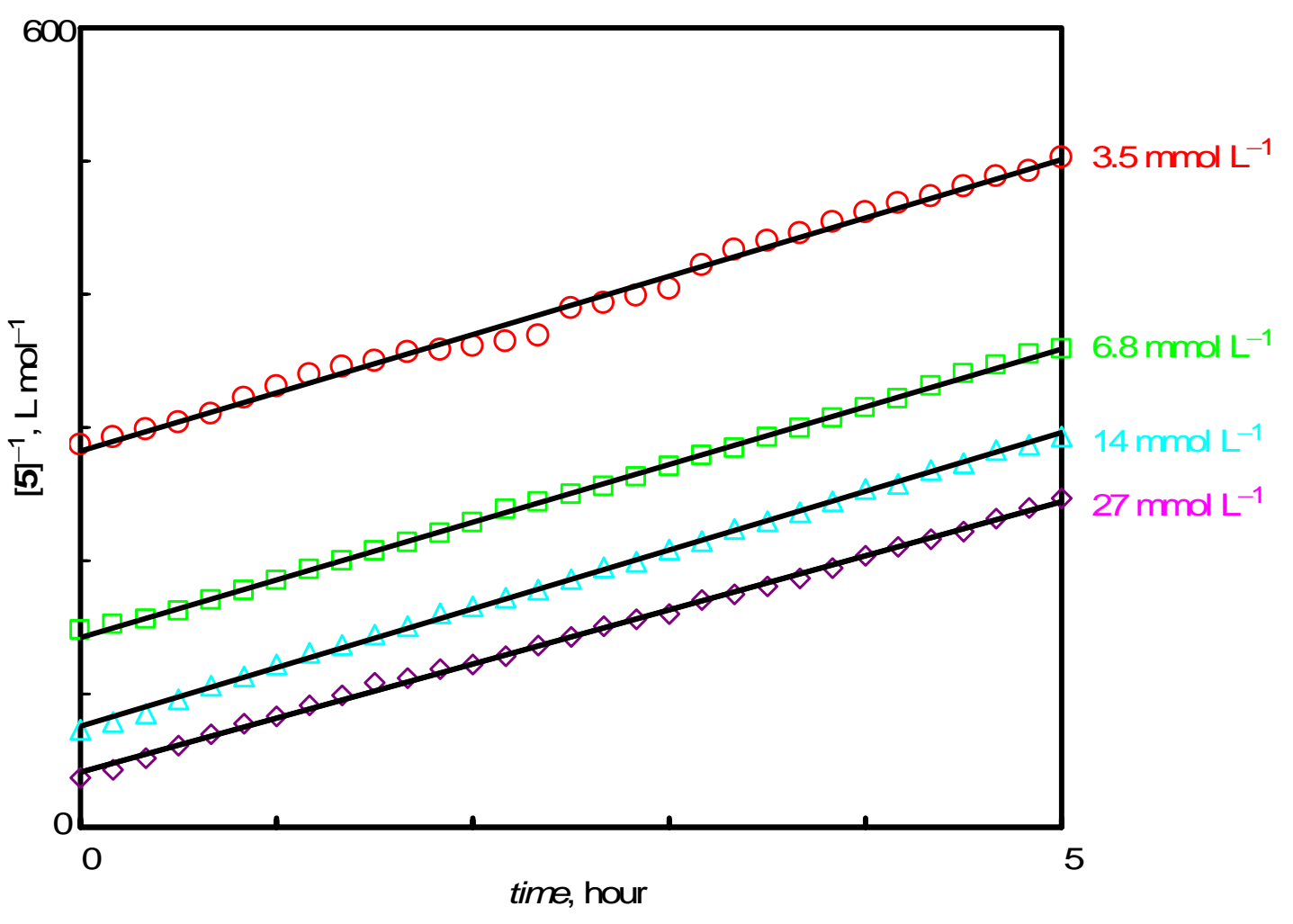

Figure S1. Plot of $[5]^{-1}$ versus Time for the Conversion of 5 at $80{ }^{\circ} \mathrm{C}$. 


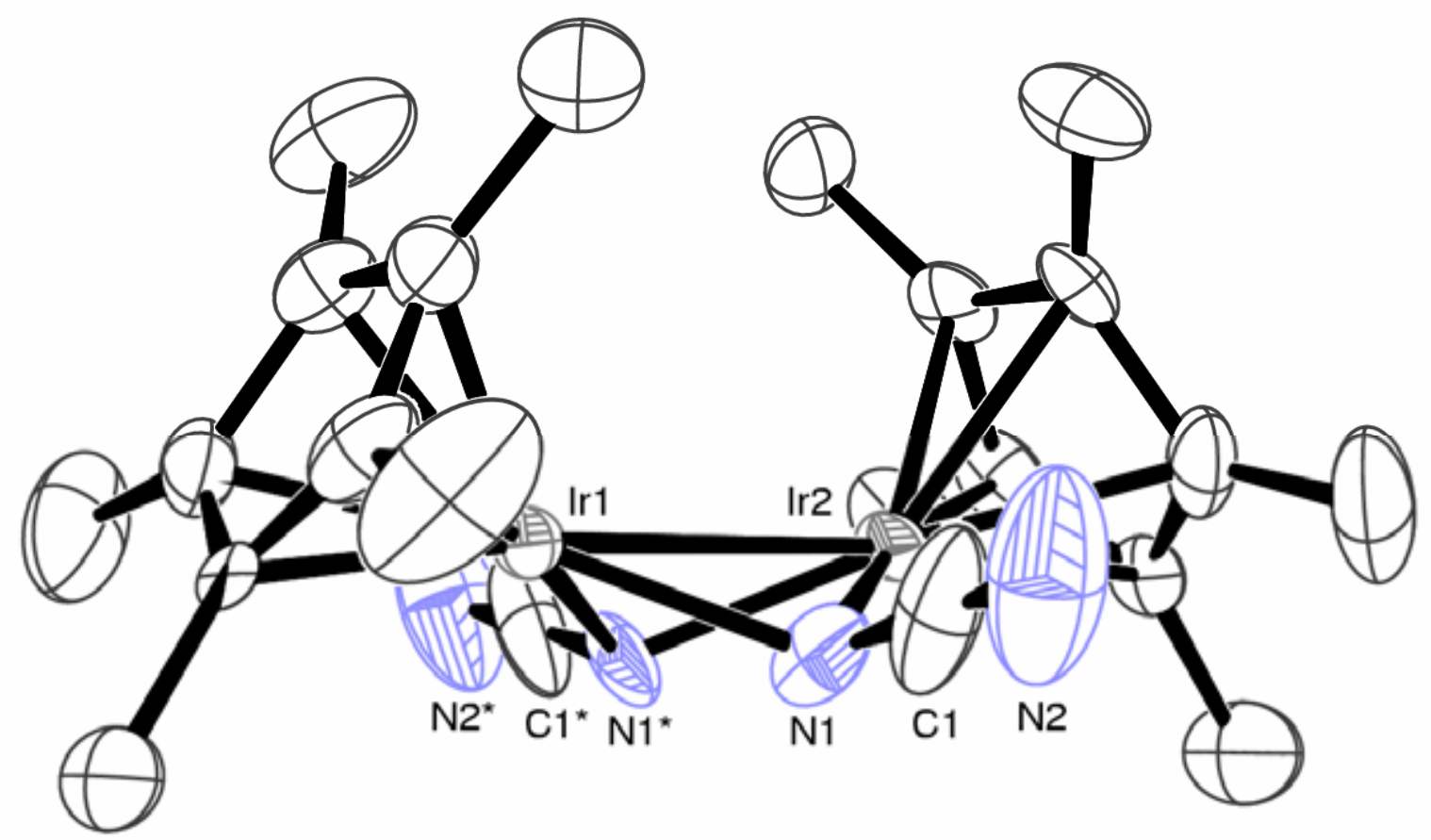

Figure S2. ORTEP Drawing of [Cp* $\left.\operatorname{Ir}\left(\mu_{2}-\mathrm{NCN}-N, N\right)\right]_{2}(5)$.

Thermal ellipsoids are shown at the 50\% probability level. Hydrogen atoms are omitted for clarity. 


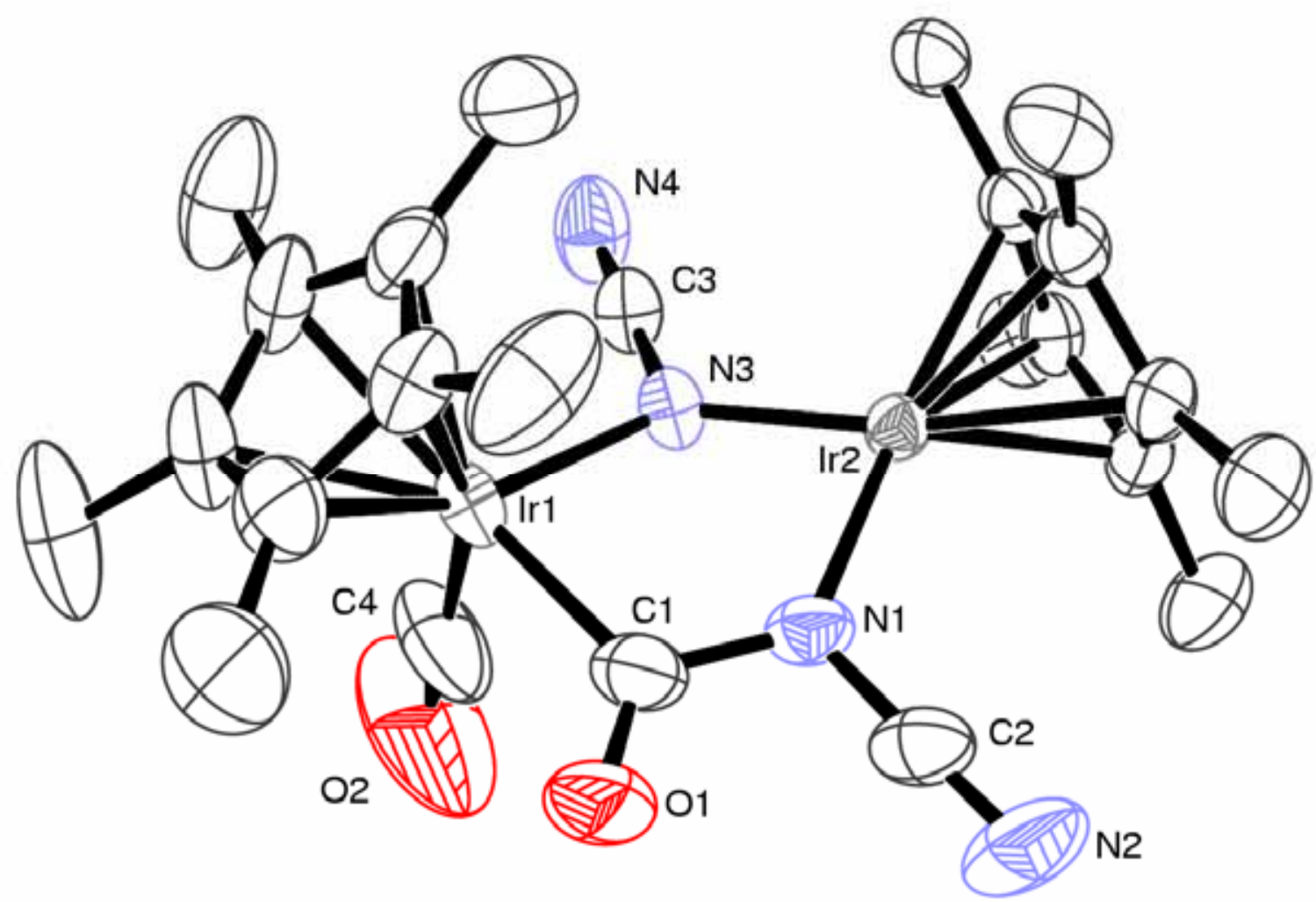

Figure S3. ORTEP Drawing of [Cp*Ir(CO) $\left(\mu_{2}-\mathrm{NCN}-N, N\right)\left(\mu_{2}\right.$-OCNCN $\left.) \operatorname{IrCp} *\right](6)$.

Thermal ellipsoids are shown at the $50 \%$ probability level. Hydrogen atoms are omitted for clarity. 


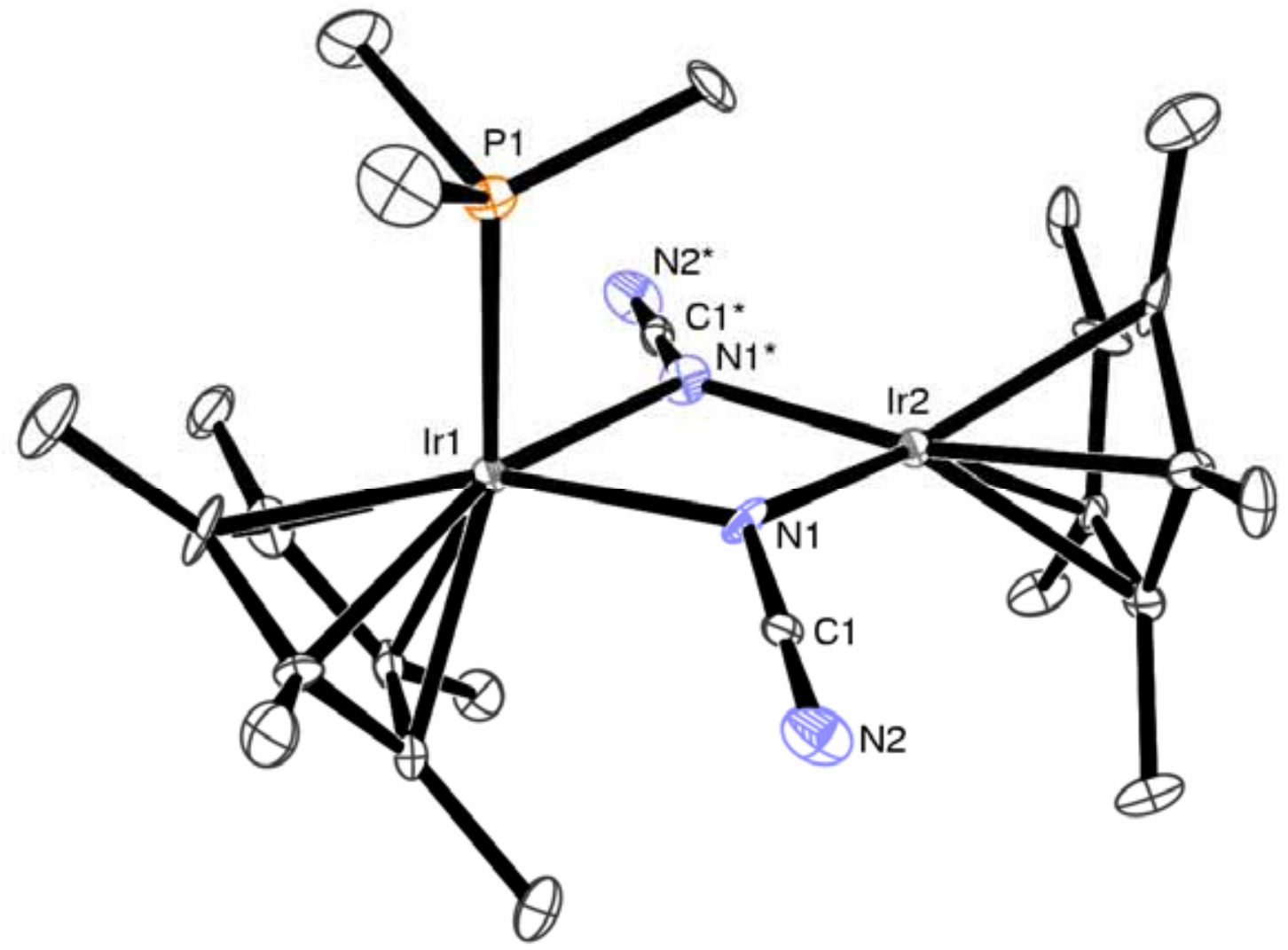

Figure S4. ORTEP Drawing of [Cp* $\left.\operatorname{Ir}\left(\mathrm{PMe}_{3}\right)\left(\mu_{2}-\mathrm{NCN}-N, N\right)_{2} \operatorname{IrCp} *\right](\mathbf{8 d})$.

Thermal ellipsoids are shown at the $50 \%$ probability level. Hydrogen atoms are omitted for clarity. 


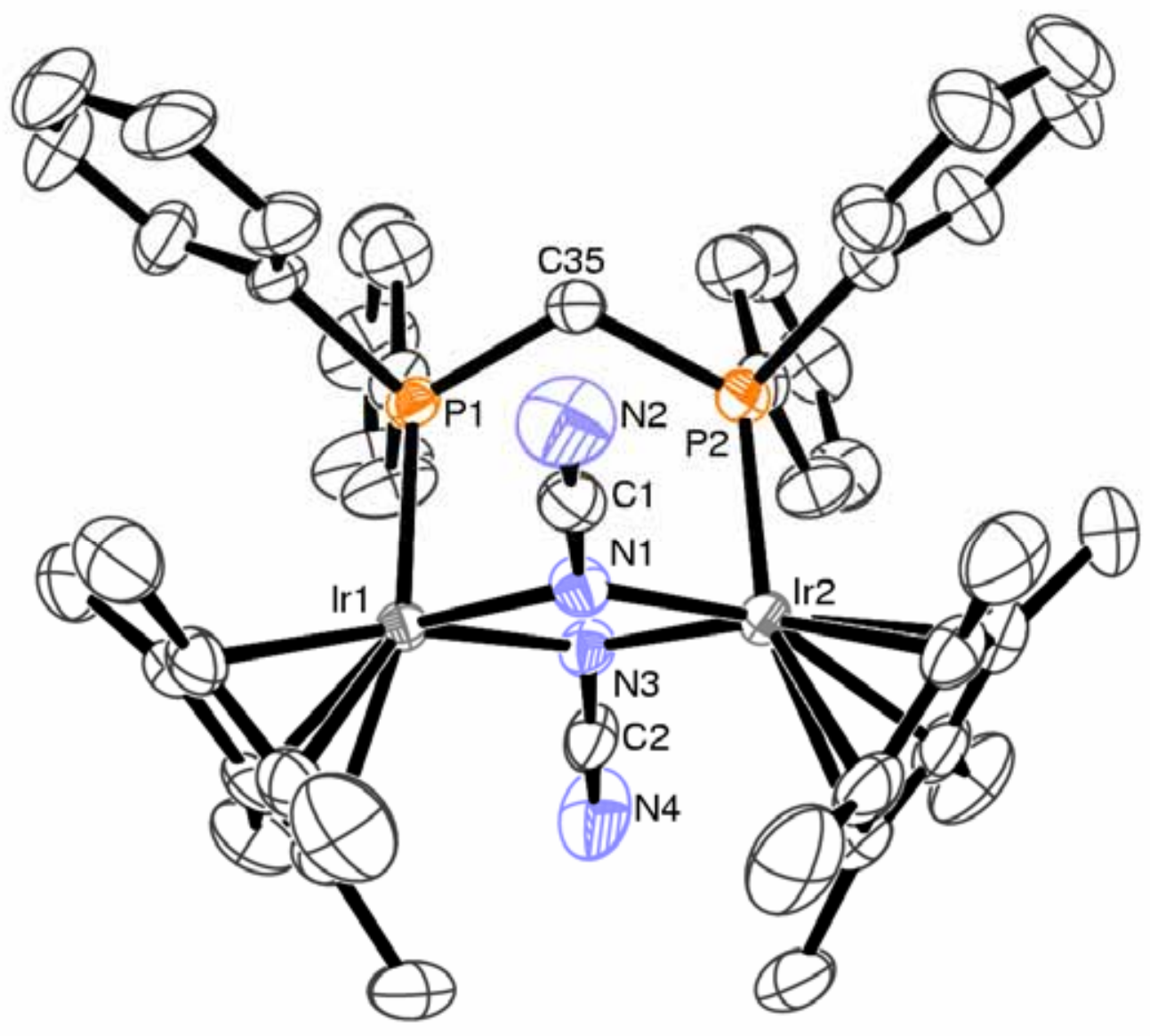

Figure S5. ORTEP Drawing of 9, the metal-containing part of [ $\left.\left\{\mathrm{Cp} * \operatorname{Ir}\left(\mu_{2}-\mathrm{NCN}-N, N\right)\right\}_{2}(\mu-\mathrm{dppm})\right] \cdot 1.5 \mathrm{C}_{6} \mathrm{H}_{6}$ $\left(\mathbf{9} \cdot 1.5 \mathrm{C}_{6} \mathrm{H}_{6}\right)$. Thermal ellipsoids are shown at the $50 \%$ probability level. Hydrogen atoms are omitted for clarity. 


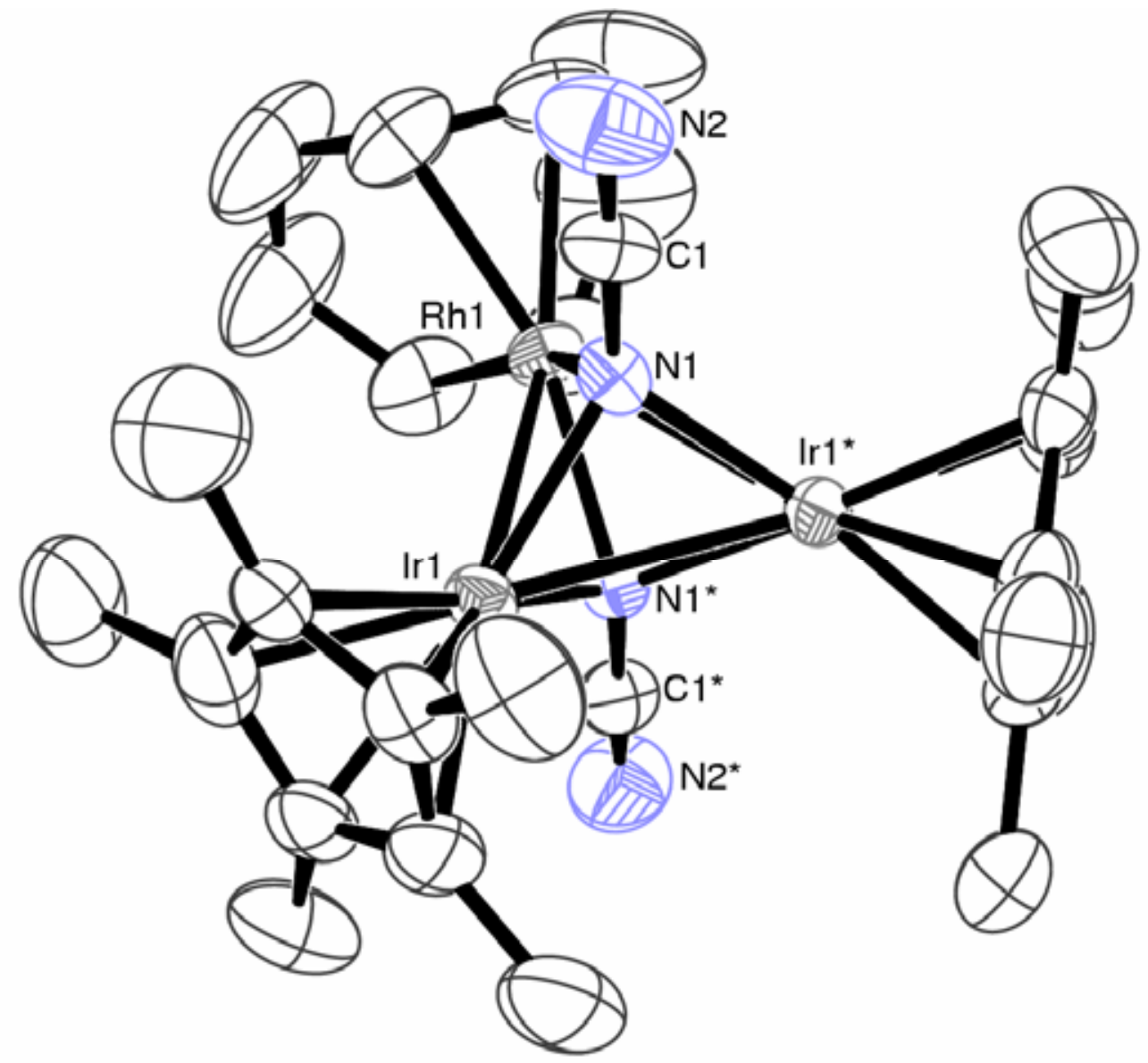

Figure S6. ORTEP Drawing of 10a, the cationic part of [(Cp*Ir $\left.)_{2}\{\mathrm{Rh}(\mathrm{cod})\}\left(\mu_{3}-\mathrm{NCN}-N, N, N\right)_{2}\right]\left(\mathrm{BPh}_{4}\right) \cdot \mathrm{CH}_{2} \mathrm{Cl}_{2}$

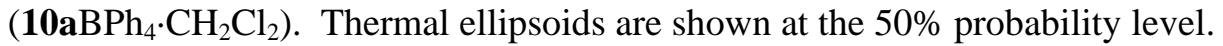
Hydrogen atoms are omitted for clarity. 